\title{
Exoplanet recycling in massive white-dwarf debris discs
}

\author{
R. van Lieshout, ${ }^{1 \star}$ Q. $\operatorname{Kral}^{1}$, S. Charnoz ${ }^{2}$, M. C. Wyatt ${ }^{1}$ and A. Shannon ${ }^{3,4,1}$ \\ ${ }^{1}$ Institute of Astronomy, University of Cambridge, Madingley Road, Cambridge CB3 OHA, UK \\ ${ }^{2}$ Institut de Physique du Globe/Université Paris Diderot/CEA/CNRS, 75005 Paris, France \\ ${ }^{3}$ Department of Astronomy E Astrophysics, The Pennsylvania State University, State College, PA 16801, USA \\ ${ }^{4}$ Center for Exoplanets and Habitable Worlds, The Pennsylvania State University, State College, PA 16802, USA
}

Accepted XXX. Received YYY; in original form ZZZ

\begin{abstract}
Several tens of white dwarfs are known to host circumstellar discs of dusty debris, thought to arise from the tidal disruption of rocky bodies originating in the star's remnant planetary system. This paper investigates the evolution of such discs if they are very massive, as may be the case if their progenitor was a terrestrial planet, moon, or dwarf planet. Assuming the discs are physically thin and flat, like Saturn's rings, their evolution is governed by PoyntingRobertson drag or viscous spreading, where the disc's effective viscosity is due to self-gravity wakes. For discs with masses $\gtrsim 10^{26} \mathrm{~g}$, located in the outer parts of the tidal disruption zone, viscous spreading dominates the evolution, and mass is transported both in- and outwards. When outwards-spreading material flows beyond the Roche limit, it coagulates into new (minor) planets in a process analogous to the ongoing formation of moonlets at the outer edge of Saturn's rings. The newly formed bodies migrate outwards by exchanging angular momentum with the disc and coalesce into larger objects through mutual collisions. Eventually, the disc's Roche-limit overflow recycles tens of percent of the original disc mass; most ends up in a single large body near 2:1 mean-motion resonance with the disc's outer edge. Hence, the recycling of a tidally disrupted super-Earth, for example, could yield an Earth-mass planet on a $\sim 10$-h orbit, located in the habitable zone for 2-to-10-Gyr-old white dwarfs. The recycling process also creates a population of smaller bodies just outside the Roche limit, which may explain the minor planets recently postulated to orbit WD $1145+017$.
\end{abstract}

Key words: accretion, accretion discs - planets and satellites: formation - planet-disc interactions - planet-star interactions - stars: individual: WD 1145+017 - white dwarfs

\section{INTRODUCTION}

Several tens of white dwarfs (WDs) are known to exhibit excess near- and mid-IR emission, revealing the presence of circumstellar dust (see Farihi 2016 for a recent review). A few of these systems also show double-peaked emission lines of metals, which indicate that metallic gas is present as well (e.g., Gänsicke et al. 2006). The estimated radial location of this circumstellar material $\left(r \lesssim 1 \mathrm{R}_{\odot}\right.$; e.g., Melis et al. 2010) is well inside the maximum stellar radius reached by the host star during its asymptotic-giant-branch phase, meaning that the material must have emerged during the WD stage. More recently, the presence of circumstellar dust in the close vicinity of a WD was confirmed by the discovery of periodic, irregularly shaped dips in the light curve of WD $1145+017$ that can be explained by transiting clouds of dust, orbiting the star at a distance of $r \approx 1 \mathrm{R}_{\odot}$ (Vanderburg et al. 2015).

WDs that exhibit IR excess also show evidence of elements heavier than helium in their atmosphere. This atmospheric metal pollution is a much more common phenomenon than the IR excess,

^ E-mail: lieshout@ast.cam.ac.uk (RvL) present at detectable levels in $1 / 4$ to $1 / 3$ of WDs (Zuckerman et al. 2010), most of which do not exhibit detectable IR excess. Given the short timescale on which metals sink out of the observable part of a WD's atmosphere (days to Myrs, depending on WD type and temperature; Koester 2009), their presence must be the result of ongoing or recent accretion. Spectroscopic analysis of polluted WDs can be used to quantify the mass accretion rates required to maintain the quickly sinking pollution and to obtain high-precision measurements of the elemental abundances of the accreted material. The latter has revealed that the composition of the accreted material in most polluted WDs broadly resembles that of the bulk Earth (Gänsicke et al. 2012; Jura \& Young 2014), although there are some interesting exceptions to this rule (e.g., Farihi et al. 2013; Xu et al. 2017).

Given these observational clues, the circumstellar material and atmospheric metal pollution are thought to originate from rocky bodies in the WD's remnant planetary system, which survives beyond the maximum asymptotic-giant-branch stellar radius $(r \gtrsim 1 \mathrm{AU}$ ). Figure 1 (top panel) summarises some of the current ideas on how mass may flow from this outer reservoir of large bodies to the WD's surface, traversing 5 orders of magnitude in radial 
distance and at least 12 in particle size. In the depicted scenario, rocky bodies are placed onto highly eccentric, star-grazing orbits, leading to their tidal disruption as they pass within the WD's Roche limit (Jura 2003). The debris produced in the tidal disruption is suggested to then spread along the orbit of the progenitor (Veras et al. 2014a), migrate towards the WD (Veras et al. 2015), and eventually form a compact circumstellar disc (Jura 2003), from which material accretes onto the WD. There are, however, still many unanswered questions regarding the exact nature of the bodies that undergo this process, the mechanism that places them on their extreme orbits, and the physics governing the subsequent steps of disc formation and accretion. In order to understand, for example, what the composition of WD pollution can tell us about exoplanetary materials, it is important to gain a better understanding of the processes governing the circumstellar environment of polluted WDs.

In the multistage process of transporting rocky material from a WD's remnant planetary system to its stellar surface, the debrisdisc phase offers a useful 'checkpoint' where theory can be compared with several sets of observations. Specifically, models of WD debris discs must be able to simultaneously explain the typical shape of the IR-excess spectral energy distribution and the mass accretion rates derived from the atmospheric metal pollution (see also Bonsor et al. 2017). A popular disc model is that of Jura (2003), who suggested that the discs are compact, opaque, and flat (much like Saturn's rings; see also Fig. 1, bottom panel, and Sect. 2.1.2), and showed that this model can reproduce the properties of the IR excess. Subsequent theoretical work on the behaviour of such discs has shown that the accretion of disc material due to PoyntingRobertson (PR) drag can explain the minimum mass accretion rates found for disc-bearing WDs (Rafikov 2011b; Bochkarev \& Rafikov 2011).

The global evolution of a WD debris disc depends critically on its total mass, but this quantity cannot be fully constrained from the observed IR excess, because the disc may be optically thick (as in the model of Jura 2003). The discs are usually assumed to contain an amount of mass comparable to that of a Solar-System asteroid $\left(\lesssim 10^{23} \mathrm{~g}\right.$ ), based on the measured masses of atmospheric pollution, which reflect the amount of mass that was accreted within the last sinking timescale (see, e.g., Fig. 6 of Veras 2016, and references therein). Nonetheless, WD debris discs that are much more massive than $10^{23} \mathrm{~g}$ may exist. For one, the distribution of pollution masses has a tail towards higher values, with the most polluted WD known, HE 0446-2531, possibly containing as much as $8 \times 10^{24} \mathrm{~g}$ (almost the mass of Pluto) of metals in its atmosphere (Friedrich et al. 2000; Girven et al. 2012). Also, pollution masses only provide a lower limit on the potential disc mass, because (1) the accretion of metals onto the WD may have been going on for much longer than one sinking timescale and (2) additional mass may remain locked up in the circumstellar debris disc.

The evolution of very massive WD debris disc has not yet been investigated in detail and it is the subject of this paper. Specifically, we consider discs that have the flat geometry proposed by Jura (2003), but contain the mass equivalent of a dwarf planet, major moon, or terrestrial planet. A possible formation scenario for such discs may be the tidal disruption of a massive rocky body (see also Sect. 4.1), but our study is ambivalent to the processes that led to the formation of the disc, taking an already-formed disc as its starting point.

A physical process that we pay special attention to is viscous spreading. This process is insignificant for the evolution of lowmass WD debris discs (Farihi et al. 2008a; Metzger et al. 2012, their Appendix A), but should be taken into account for the high-mass

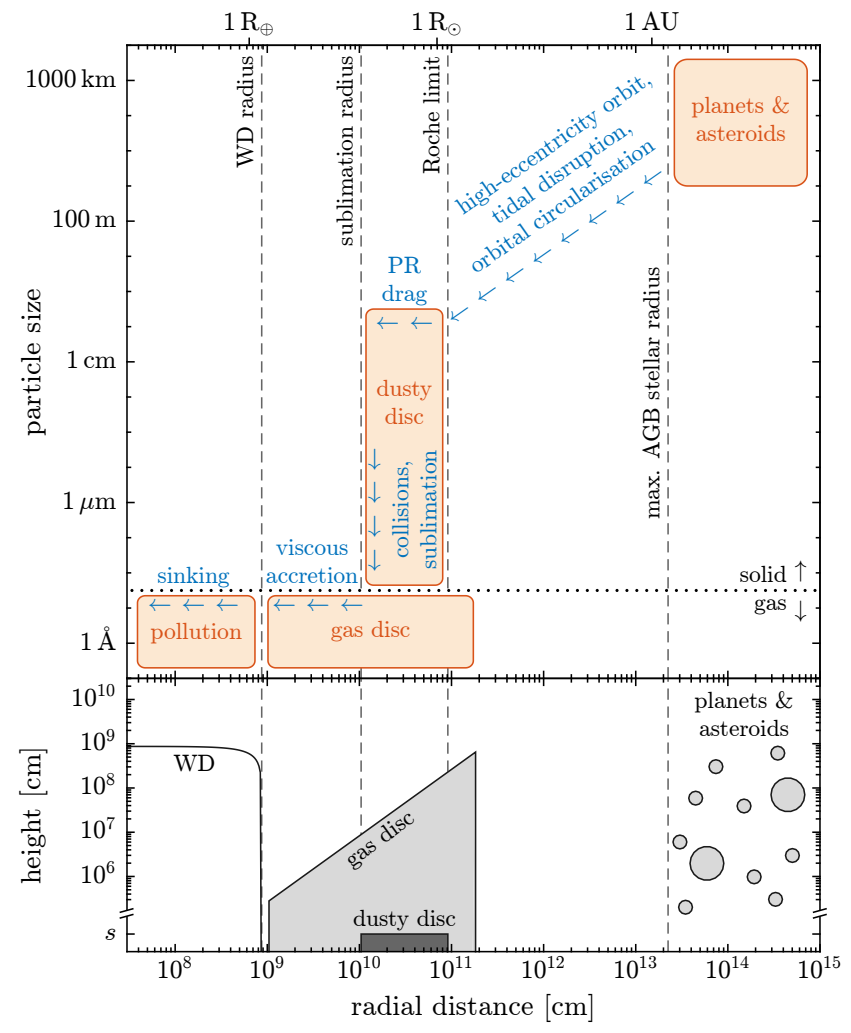

Figure 1. Overview of a possible model for the circumstellar environment of polluted WDs and the processes leading to rocky material reaching the star. Top panel: Main components of a polluted WD system (red boxes) and the dominant mass flow through the system (blue arrows). Bottom panel: Geometry corresponding to this model. Note the break in the vertical axis; $s$ denotes the typical radius of debris particles in the dusty disc.

discs that we consider here, because its effects become stronger at higher surface densities. From planetary-ring studies, it is known that the viscous spreading of a tidal disc can result in the formation of gravitationally bound objects at the disc's outer edge, specifically explaining the presence and properties of the small moons located close to the outer edge of Saturn's rings (Charnoz et al. 2010; Crida \& Charnoz 2012, hereafter CC12). We find that a similar process may occur in massive WD debris discs. Thus, a large rocky body (such as a planet) that undergoes a tidal disruption around a WD, forming a massive debris disc, can be partially 'recycled' into a set of second-generation (minor) planets (cf. Bear \& Soker 2015). This recycling mechanism may be the origin of the close-in, massive bodies that have been suggested to orbit WD $1145+017$ to explain some aspects of this WD's peculiar transits (Vanderburg et al. 2015; Gänsicke et al. 2016; Rappaport et al. 2016).

The rest of this paper is organised as follows. In Sect. 2, we use analytical arguments to investigate the properties and evolution of massive WD debris discs, and of the second-generation (minor) planets they may produce. In Sect. 3, these findings are checked and expanded on using a numerical model adapted from studies of planetary ring systems. In Sect. 4, we discuss various aspects of the exoplanet-recycling scenario, including the prospects of testing its predictions observationally; whether the scenario can help explain some of the unresolved properties of the transits of WD 1145+017; and the potential of forming second-generation exoplanets in this manner around other astrophysical objects, such as pulsars, hot sub- 
Table 1. Definition of symbols.

\begin{tabular}{|c|c|c|}
\hline Symbol & Meaning & See also \\
\hline$A$ & Shearing rate of adjacent annuli in the disc & Eq. (A2) \\
\hline$c$ & Speed of light & \\
\hline$C_{\mathrm{R}}$ & Prefactor in Roche-limit equation & Eq. (1) \\
\hline$C_{\text {visc }}$ & Self-gravitational viscosity correction factor & Eq. (13) \\
\hline$D_{\text {tr }}$ & Full transit duration & Eq. (49) \\
\hline $\begin{array}{l}f_{\varphi, \mathrm{PR}} \\
G\end{array}$ & $\begin{array}{l}\text { Azimuthal PR-drag force per unit area } \\
\text { Gravitational constant }\end{array}$ & Eq. (A4) \\
\hline$L_{\star}$ & WD luminosity & Sect. 2.3.2 \\
\hline$m$ & Grain mass & Sect. 2.1.2 \\
\hline$M_{\star}$ & WD mass & \\
\hline$M_{\text {crit }}$ & $\begin{array}{l}\text { Disc mass above which viscous spreading } \\
\text { dominates PR drag }\end{array}$ & Eq. (18) \\
\hline$M_{\text {disc }}$ & Initial disc mass & Eq. (3) \\
\hline$M_{\text {form }}$ & $\begin{array}{l}\text { Disc mass above which viscosity spreads } \\
\text { disc to beyond Roche limit }\end{array}$ & Eq. (20) \\
\hline$M_{\mathrm{p}}$ & Mass of (minor) planet & Sect. 2.4.3 \\
\hline$M_{\text {recyc }}$ & Total mass in newly formed (minor) planets & Eq. (45) \\
\hline$M_{\mathrm{T}}$ & $\begin{array}{l}\text { Toomre mass, i.e., mass enclosed in a circu- } \\
\text { lar portion of the disc with diameter } \lambda_{\mathrm{T}}\end{array}$ & Eq. (27) \\
\hline$\dot{M}_{\text {out }}$ & $\begin{array}{l}\text { Outward mass flow rate at the Roche limit } \\
\text { caused by viscous spreading }\end{array}$ & Eq. (26) \\
\hline$\dot{M}_{\mathrm{PR}}$ & PR-drag-induced inward mass flow rate & Eq. (15) \\
\hline$n$ & Number of (minor) planets & Sect. 2.4.3 \\
\hline$N_{\text {in }}$ & Number of in-transit photometry points & Eq. (50) \\
\hline$p$ & Integer identifying mean-motion resonance & Sect. 2.4.6 \\
\hline$p_{\text {tr }}$ & Geometric transit probability & Eq. (47) \\
\hline$P_{2: 1}$ & Keplerian orbital period at $r_{2: 1}$ & Eq. (46) \\
\hline$P_{\mathrm{R}}$ & Keplerian orbital period at Roche limit & Eq. (2) \\
\hline$q$ & Planet-to-WD mass ratio & Eq. (29) \\
\hline$q_{0}$ & $q$ at start of pyramidal regime & Eq. (39) \\
\hline$q_{\mathrm{c}}$ & $q$ at end of continuous regime & Eq. (32) \\
\hline$q_{\mathrm{d}}$ & $q$ at end of discrete regime & Eq. (33) \\
\hline$Q_{\mathrm{T}}$ & Toomre's gravitational stability parameter & Eq. (7) \\
\hline$r$ & Radial distance & \\
\hline$r_{0}$ & Initial (central) radius of the disc & \\
\hline$r_{2: 1}$ & $\begin{array}{l}\text { Radius at which the } 2: 1 \mathrm{MMR} \text { of an orbiting } \\
\text { body coincides with the Roche limit }\end{array}$ & Eq. (40) \\
\hline$r_{\text {subl }}$ & Sublimation radius & Sect. 2.1.2 \\
\hline$r_{\mathrm{H}}$ & Mutual Hill radius of disc particles & Eq. (12) \\
\hline$r_{\mathrm{H}}^{*}$ & $r_{\mathrm{H}}$ scaled to the sum of the particles' radii & Eq. (12) \\
\hline$r_{\mathrm{R}}$ & Roche limit & Eq. (1) \\
\hline$R_{\star}$ & WD radius & \\
\hline$R_{\mathrm{p}}$ & Radius of (minor) planet & Sect. 2.4.3 \\
\hline$s$ & Grain radius & Sect. 2.1.2 \\
\hline$t$ & Time & \\
\hline$t_{\mathrm{c}}$ & $\begin{array}{l}\text { Time interval between formation of two } \\
\text { bodies at }\left\{\Delta_{\mathrm{c}}, q_{\mathrm{c}}\right\}\end{array}$ & Eq. (34) \\
\hline$t_{\text {crit }}$ & $\begin{array}{l}\text { Disc evolution time at transition from } \\
\text { viscous spreading to PR drag }\end{array}$ & Eq. (22) \\
\hline$t_{\text {cool }}$ & WD cooling age & Sect. 3.1.2 \\
\hline$t_{\mathrm{d}}$ & $\begin{array}{l}\text { Time interval between formation of two } \\
\text { bodies at }\left\{\Delta_{\mathrm{d}}, q_{\mathrm{d}}\right\}\end{array}$ & Eq. (35) \\
\hline$t_{\text {disc }}$ & Disc lifetime, i.e., until no disc mass remains & Sect. 2.3.5 \\
\hline$t_{\text {evol }}$ & $\begin{array}{l}\text { Disc evolution timescale, i.e., on which } \\
\text { changes in local surface density occur }\end{array}$ & Fig. 3 \\
\hline$t_{\mathrm{PR}}$ & PR-drag disc-evolution timescale & Eq. (16) \\
\hline$t_{\text {start }}$ & Time when Roche-limit overflow commences & Sect. 2.4.1 \\
\hline$t_{\mathrm{T}}$ & $\begin{array}{l}\text { Time interval between formation of two } \\
\text { bodies of mass } M_{\mathrm{T}}\left(r_{\mathrm{R}}\right)\end{array}$ & Eq. (28) \\
\hline$t_{\mathrm{visc}}$ & Viscous disc-evolution timescale & Eq. (14) \\
\hline$T_{\star}$ & WD effective temperature & \\
\hline $\mathcal{T}$ & $\begin{array}{l}\text { Disc's viscous timescale at Roche limit } \\
\text { scaled to local orbital period }\end{array}$ & Eq. (30) \\
\hline$Z$ & Auxiliary variable for discrete regime & Eq. (33) \\
\hline$Z_{0}$ & Numerical constant, $Z_{0} \approx 0.682$ & Eq. (33) \\
\hline
\end{tabular}

Table 1 - continued

\begin{tabular}{|c|c|c|}
\hline Symbol & Meaning & See also \\
\hline$\alpha, \beta$ & $\begin{array}{l}\text { Power-law indices for distance and sur- } \\
\text { face-density dependence of viscosity }\end{array}$ & Sect. 2.3.1 \\
\hline$\Gamma_{\mathrm{PR}}$ & PR drag torque & Eq. (A5) \\
\hline$\Gamma_{\text {visc }}$ & Viscous torque & Eq. (A2) \\
\hline$\delta$ & Initial scaled disc width & Eq. (3) \\
\hline$\delta_{\mathrm{tr}}$ & Transit depth & Eq. (48) \\
\hline$\Delta$ & Scaled distance beyond Roche limit & Eq. (29) \\
\hline$\Delta_{0}$ & $\Delta$ at start of pyramidal regime & Eq. (38) \\
\hline$\Delta_{2: 1}$ & $\begin{array}{l}\Delta \text { at which the } 2: 1 \text { MMR of an orbiting body } \\
\text { coincides with the Roche limit }\end{array}$ & Eq. (41) \\
\hline$\Delta_{\mathrm{c}}$ & $\Delta$ at end of continuous regime & Eq. (32) \\
\hline$\Delta_{\mathrm{d}}$ & $\Delta$ at end of discrete regime & Eq. (33) \\
\hline$\zeta$ & Impinging angle of stellar radiation & Eq. (5) \\
\hline$\eta$ & $\begin{array}{l}\text { Numerical coefficient in Toomre's gravi- } \\
\text { tational stability criterion, } \eta \approx 3.36\end{array}$ & Eq. (7) \\
\hline$\lambda_{\mathrm{T}}$ & Toomre's critical wavelength & Eq. (8) \\
\hline$v$ & Effective kinetic viscosity & Eq. (13) \\
\hline$\rho_{\star}$ & WD mean density & Eq. (1) \\
\hline$\rho_{\mathrm{d}}$ & Material density of disc particles & Sect. 2.1.2 \\
\hline$\sigma_{r}$ & Radial velocity dispersion & Eq. (9) \\
\hline$\sigma_{\mathrm{SB}}$ & Stefan-Boltzmann constant & \\
\hline$\sigma_{\text {phot }}$ & $\begin{array}{l}\text { Photometric precision on a single } \\
\text { measurement in a light curve }\end{array}$ & Eq. (50) \\
\hline$\Sigma$ & Disc's surface mass density & Eq. (3) \\
\hline$\Sigma_{\text {crit }}$ & $\begin{array}{l}\text { Disc's surface density above which viscous } \\
\text { spreading dominates PR drag }\end{array}$ & Eq. (17) \\
\hline$\Sigma_{\text {form }}$ & $\begin{array}{l}\text { Disc's surface density above which viscosity } \\
\text { spreads out disc to beyond Roche limit }\end{array}$ & Fig. 6 \\
\hline$\Sigma_{\mathrm{R}}$ & Disc's surface density at the Roche limit & Sect. 2.4.1 \\
\hline$\Sigma_{\text {visc }}$ & surface-density profile with constant $t_{\text {visc }}$ & Sect. 2.3.1 \\
\hline$\tau_{\perp}$ & Disc's vertical optical depth & Eq. (4) \\
\hline$\tau_{\|}$ & Disc's optical depth to radial stellar radiation & Eq. (6) \\
\hline$\phi_{r}$ & Momentum-transfer efficiency for PR drag & Eq. (A4) \\
\hline$\Omega_{\mathrm{K}}$ & Keplerian angular frequency & Sect. 2.2 \\
\hline
\end{tabular}

dwarfs, and brown dwarfs. We finish with a set of conclusions in Sect. 5. For reference, a list of symbol definitions used in this paper is given in Table 1.

\section{ANALYTICAL CONSIDERATIONS}

In this section, we analytically investigate the evolution of massive WD debris discs and the second-generation planets that they may produce. We start by introducing key concepts and assumptions, and specifying a prescription for the disc (Sect. 2.1). Next, we assess the gravitational stability of WD debris discs, which is important because it affects the disc's effective viscosity (Sect. 2.2). We then investigate the evolution of these discs under the effects of viscous spreading and Poynting-Robertson drag, and determine what discs are able to spawn a new generation of (minor) planets (Sect. 2.3). Lastly, we explore what the basic properties of these newly formed bodies might be (Sect. 2.4). A brief summary of our analytical findings is included at the end of the section (Sect. 2.5).

\subsection{Preliminaries}

\subsubsection{The Roche limit}

A central concept in this study is the Roche limit: the distance within which self-gravity cannot hold together a low-mass body 
against the tidal forces and Keplerian shear caused by a large central mass. ${ }^{1}$ For material with an internal density of $\rho_{\mathrm{d}}$, orbiting a WD of radius $R_{\star}$, mass $M_{\star}$, and mean density $\rho_{\star}$, it is given by (e.g., Roche 1849; Chandrasekhar 1969)

$$
\begin{aligned}
r_{\mathrm{R}} & =C_{\mathrm{R}}\left(\frac{\rho_{\star}}{\rho_{\mathrm{d}}}\right)^{1 / 3} R_{\star}=C_{\mathrm{R}}\left(\frac{3}{4 \pi} \frac{M_{\star}}{\rho_{\mathrm{d}}}\right)^{1 / 3} \\
& \approx 1.3 \mathrm{R}_{\odot}\left(\frac{C_{\mathrm{R}}}{2.0}\right)\left(\frac{M_{\star}}{0.6 \mathrm{M}_{\odot}}\right)^{1 / 3}\left(\frac{\rho_{\mathrm{d}}}{3 \mathrm{~g} \mathrm{~cm}^{-3}}\right)^{-1 / 3} .
\end{aligned}
$$

Here, the prefactor $C_{\mathrm{R}}$ is a constant between about 1.3 and 2.9, which depends on the strength, shape, orientation, and spin state of the low-mass body (see Harris 1996; Holsapple \& Michel 2006, 2008). The classical value found by Roche (1849) is $C_{\mathrm{R}} \approx 2.456$, valid for homogeneous, synchronously rotating, incompressible fluid bodies. We keep $C_{\mathrm{R}}$ as a free parameter for most of our analysis, but when needed we take $C_{\mathrm{R}}=2.0$ as a fiducial value. This value is valid for 'rubble piles' (i.e., particle aggregates held together solely by gravity) whose shape is such that they fill their Roche lobe (Porco et al. 2007; $\gamma \approx 1.6$ in their notation). It can explain the observed densities of Saturn's innermost moonlets (Porco et al. 2007) and is roughly consistent with the observed architectures of the Saturnian and Uranian systems, where the Roche limit marks the boundary between quasi-continuous particle rings and discrete moons (Tiscareno et al. 2013).

Combining Eq. (1) with Kepler's third law gives the orbital period of a body located at the Roche limit

$P_{\mathrm{R}}=\sqrt{\frac{3 \pi C_{\mathrm{R}}^{3}}{G \rho_{\mathrm{d}}}} \approx 5.4 \mathrm{~h}\left(\frac{C_{\mathrm{R}}}{2.0}\right)^{3 / 2}\left(\frac{\rho_{\mathrm{d}}}{3 \mathrm{~g} \mathrm{~cm}^{-3}}\right)^{-1 / 2}$.

It is worth noting that the dependence on the central mass has been eliminated here.

\subsubsection{Basic properties of the particle disc}

We consider geometrically thin, flat, compact, axisymmetric discs, made up of solid debris particles, similar in many respects to Saturn's rings. With 'geometrically thin' we mean that the disc's thickness is considerably smaller than the WD's diameter, while 'flat' means that the thickness does not vary with radius. The disc may converge to this state through successive energy-dissipating collisions between the constituent particles (e.g., Brahic 1977; Cuzzi et al. 1979, but see Kenyon \& Bromley 2017a for a contrasting view). With 'compact' we mean that the disc is located within the Roche limit $r_{\mathrm{R}}$, as expected for a disc resulting from a tidal disruption followed by orbital shrinking and circularisation of the debris. Our study will concentrate mostly (but not exclusively) on discs located in the outer parts of the tidal disruption zone $\left(0.5 \lesssim r / r_{\mathrm{R}}<1\right)$. Debris discs may preferentially be found here, because the outer parts of the tidal disruption zone have a larger cross-section to incoming bodies than the inner parts.

For simplicity, we assume that the particles making up the disc are all identical spheres with radius $s$, internal density $\rho_{\mathrm{d}}$, and mass $m=4 \pi \rho_{\mathrm{d}} s^{3} / 3$. We will use $\rho_{\mathrm{d}}=3 \mathrm{~g} \mathrm{~cm}^{-3}$ as a fiducial value for

\footnotetext{
1 Sometimes, the term 'Roche limit' is used to refer strictly to the incompressible-fluid case with $C_{\mathrm{R}} \approx 2.456$ in Eq. (1). We use the term more generally to mean the radial distance inside of which tides and shear cause an approaching secondary body to break up, or prevent a ring of particles from accumulating into a larger body (Holsapple \& Michel 2006).
}

the density, in line with the inferred rocky composition of WD atmospheric pollution (Jura \& Young 2014). Regarding the typical sizes of particles in WD debris discs, there are only a few (relatively weak) observational constraints: Firstly, several WDs exhibit $10 \mu \mathrm{m}$ silicate emission features, indicating the presence of (at least some) $s \sim 1 \mu \mathrm{m}$ dust (e.g. Jura et al. 2007a, 2009a). Secondly, Graham et al. (1990) put a rough upper limit of $s \lesssim 1 \mathrm{~m}$ on the size of the particles orbiting G29-38 based on their detection of IR pulsations (interpreted as the WD's optical pulsations that are reprocessed into the IR by the circumstellar dust, putting an upper limit on the thermal inertia of the dust grains). These two results bracket grain sizes of $s \sim 1 \mathrm{~cm}$, which we will use as a fiducial value when one is needed. Additional motivation for using this value comes from the analogy with Saturn's rings, in which particles of a few $\mathrm{cm}$ are the most abundant (e.g., Cuzzi et al. 2009). As we will see later, however, most of the conclusions made in this study ultimately turn out to be independent of grain size, as long as the particles are not too large (see Sects. 2.3.3 and 3.1.2).

Our study is focussed specifically on massive discs, with masses $M_{\text {disc }}$ roughly ranging from that of Ceres $\left(\sim 10^{24} \mathrm{~g}\right)$ to that of a super-Earth $\left(10^{28}\right.$ to $\left.10^{29} \mathrm{~g}\right)$. Assuming that the disc initially constitutes a relatively narrow annulus at radius $r$, its surface mass density can be estimated as

$\Sigma=\frac{M_{\text {disc }}}{2 \pi \delta r^{2}} \approx 3 \times 10^{4} \mathrm{~g} \mathrm{~cm}^{-2}\left(\frac{\delta}{0.1}\right)^{-1}\left(\frac{M_{\text {disc }}}{10^{26} \mathrm{~g}}\right)\left(\frac{r}{1 \mathrm{R}_{\odot}}\right)^{-2}$,

where $\delta$ is a dimensionless factor that characterises the disc's width (i.e., the disc width is roughly $\delta \times r$ ). Using the assumption of equal-sized, spherical grains, we can write the vertical optical depth of the disc as

$\tau_{\perp}=\frac{3}{4} \frac{\Sigma}{\rho_{\mathrm{d}} s} \approx 3 \times 10^{3}\left(\frac{\rho_{\mathrm{d}}}{3 \mathrm{~g} \mathrm{~cm}^{-3}}\right)^{-1}\left(\frac{s}{1 \mathrm{~cm}}\right)^{-1}\left(\frac{\Sigma}{10^{4} \mathrm{~g} \mathrm{~cm}^{-2}}\right)$.

This also assumes that the grains can be treated using the geometrical-optics approximation, which is the case if the particles are much larger than the wavelength of the radiation under consideration. Since the disc is assumed to be vertically much smaller than the WD, but horizontally much larger, stellar radiation impinges on the disc at a shallow angle $\zeta$. Following the 'lamp-post illumination model' of Rafikov (2011b; see also Friedjung 1985), this impinging angle is

$\zeta \simeq \frac{4}{3 \pi} \frac{R_{\star}}{r} \approx 0.3^{\circ}\left(\frac{R_{\star}}{0.0125 \mathrm{R}_{\odot}}\right)\left(\frac{r}{1 \mathrm{R}_{\odot}}\right)^{-1}$,

and the disc's optical depth to the impinging stellar radiation is

$$
\begin{aligned}
\tau_{\|}=\frac{\tau_{\perp}}{\zeta} \simeq \frac{9 \pi}{16} \frac{\Sigma}{s \rho_{\mathrm{d}}} \frac{r}{R_{\star}} \approx & 5 \times 10^{5}\left(\frac{R_{\star}}{0.0125 \mathrm{R}_{\odot}}\right)\left(\frac{\rho_{\mathrm{d}}}{3 \mathrm{~g} \mathrm{~cm}^{-3}}\right)^{-1} \\
& \times\left(\frac{s}{1 \mathrm{~cm}}\right)^{-1}\left(\frac{\Sigma}{10^{4} \mathrm{~g} \mathrm{~cm}^{-2}}\right)\left(\frac{r}{1 \mathrm{R}_{\odot}}\right)^{-1} .
\end{aligned}
$$

This shows that the massive discs considered in this study can be assumed to be opaque to the stellar radiation.

\subsubsection{The sublimation radius and the gaseous disc}

Very close to the WD, disc temperatures will become high enough for the dust material to sublimate. The distance $r_{\text {subl }}$ at which this happens depends on the WD temperature, disc composition, and ambient gas pressure. For simplicity, we use a fixed value of $r_{\text {subl }}=$ $0.15 R_{\odot}$, roughly consistent with theoretical expectations and observational estimates (e.g., Jura et al. 2007b; Rafikov \& Garmilla 
2012). Inside this sublimation radius, the solid debris quickly turns to gas, which is assumed to then rapidly accrete onto the WD via a gaseous viscous accretion disc (e.g., Metzger et al. 2012).

In this work we mostly ignore the possible back-reaction of the gas that spreads outwards from $r_{\text {subl }}$ on the particle disc. Rafikov (2011a) and Metzger et al. (2012) have found that the drag due to this gas may cause a run-away accretion of the particle disc onto the WD. Whether this process happens, however, depends critically on the strength of the positive feedback of the gas on the solid disc (called $\mathcal{F}$ by Rafikov 2011a and Metzger et al. 2012), which is highly uncertain because it is controlled by ill-constrained parameters of the gas disc such as its viscosity $(\alpha)$ and the critical Reynolds number above which the gas flow becomes fully turbulent $\left(R e_{*}\right)$. Ignoring gas drag simply corresponds to assuming a low feedback $(\mathcal{F}<1)$. Furthermore, gas outside the sublimation radius is expected to rapidly condense onto the solid particle disc, which may hinder the run-away process. Nevertheless, we briefly discuss the possibility of disc destruction due to gas-drag-induced run-away accretion in Sect. 4.2.

\subsection{Gravitational stability of the disc}

To evaluate the gravitational stability of massive WD debris discs, we follow the analyses of Daisaka et al. (2001) and Schmidt et al. (2009) for planetary rings. Given the (presumed) similarity between WD debris discs and planetary rings, their reasoning can be directly applied here.

The gravitational stability of a differentially rotating disc against axisymmetric perturbations can be assessed using Toomre's parameter $Q_{\mathrm{T}}$ (Toomre 1964), which for a Keplerian particle disc is

$Q_{\mathrm{T}}=\frac{\sigma_{r} \Omega_{\mathrm{K}}}{\eta G \Sigma}, \quad \eta \approx 3.36$.

Here, $\sigma_{r}$ is the radial velocity dispersion of the disc particles, $\Omega_{\mathrm{K}}=\sqrt{G M_{\star} / r^{3}}$ is the Keplerian angular frequency, and $G$ is the gravitational constant. Gravitational instabilities occur for $Q_{\mathrm{T}} \lesssim 2$ and have a typical length scale of the order of Toomre's critical wavelength (Toomre 1964; Julian \& Toomre 1966)

$$
\begin{aligned}
\lambda_{\mathrm{T}} & =\frac{4 \pi^{2} G \Sigma}{\Omega_{\mathrm{K}}^{2}}=3 \pi\left(\frac{C_{\mathrm{R}} r}{r_{\mathrm{R}}}\right)^{3} \frac{\Sigma}{\rho_{\mathrm{d}}} \\
& \approx 2.5 \mathrm{~km}\left(\frac{C_{\mathrm{R}}}{2.0}\right)^{3}\left(\frac{\rho_{\mathrm{d}}}{3 \mathrm{~g} \mathrm{~cm}^{-3}}\right)^{-1}\left(\frac{\Sigma}{10^{4} \mathrm{~g} \mathrm{~cm}^{-2}}\right)\left(\frac{r / r_{\mathrm{R}}}{1}\right)^{3} .
\end{aligned}
$$

To determine the correct expression for the radial velocity dispersion $\sigma_{r}$, it is important to determine what processes govern the motions of the particles. In the discs considered here, low-velocity, bouncing collisions are frequent and they set the base level for the particle motions. These physical impacts regulate the velocity dispersion to be of the order of the difference in orbital velocities between radially adjacent particles $2 s \Omega_{\mathrm{K}}$ (Brahic 1977; Wisdom $\&$ Tremaine 1988; Salo 1995). The velocity dispersion can be enhanced above this base level by close gravitational encounters between the disc particles, which yield motions of the order of their mutual escape speed $\sqrt{2 G m / s}$ (e.g., Salo 1995; Ohtsuki 1999). If the velocity dispersion due to physical impacts is already higher than this, however, the effect of gravitational encounters will be negligible. Thus, the radial velocity dispersion can be expressed as

$\sigma_{r} \sim \max \left[2 s \Omega_{\mathrm{K}}, \sqrt{2 G m / s}\right]$.

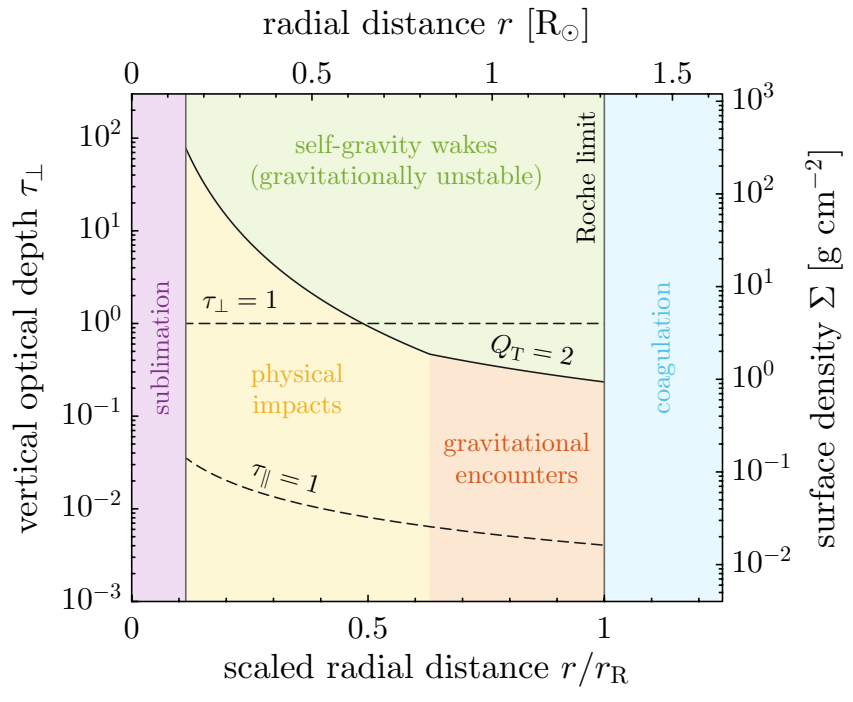

Figure 2. Gravitational stability criterion for a particle disc (solid line; Eq. (10)), as a function of radial distance $r$ scaled to the Roche limit $r_{\mathrm{R}}$, assuming a Roche prefactor of $C_{\mathrm{R}}=2.0$. Coloured shadings demarcate parts of parameter space in which different processes dominate (cf. Fig. 14.7 of Schmidt et al. 2009). Dashed lines indicate where the disc becomes optically thick vertically $\left(\tau_{\perp}=1\right)$ and optically thick to the impinging stellar radiation $\left(\tau_{\|}=1\right.$; Eq. (6); valid for the top axis and a stellar radius of $R_{\star}=0.0125 \mathrm{R}_{\odot}$ ). Vertical lines mark the dust sublimation radius (at $r_{\text {subl }}=0.15 \mathrm{R}_{\odot}$ ) and the Roche limit. The absolute distances indicated along the top axis assume a stellar mass of $M_{\star}=0.6 \mathrm{M}_{\odot}$, a material density of $\rho_{\mathrm{d}}=3 \mathrm{~g} \mathrm{~cm}^{-3}$, and $C_{\mathrm{R}}=2.0$ (see Eq. (1)). The surface densities indicated along the right-hand-side axis assume $\rho_{\mathrm{d}}=3 \mathrm{~g} \mathrm{~cm}^{-3}$ and a particle size of $s=1 \mathrm{~cm}$ (see Eq. (4)).

The transition between the two regimes in this equation occurs at $r / r_{\mathrm{R}}=2^{1 / 3} / C_{\mathrm{R}} \approx 0.63\left(C_{\mathrm{R}} / 2.0\right)^{-1}$.

Inserting the expression for $\sigma_{r}$ into Eq. (7), together with $Q_{\mathrm{T}} \lesssim$ 2, and rewriting the result using Eqs. (1) and (4) gives the relatively simple gravitational stability criterion

$\tau_{\perp} \gtrsim \max \left[\frac{\pi}{\eta}\left(\frac{r_{\mathrm{R}}}{C_{\mathrm{R}} r}\right)^{3}, \sqrt{\frac{1}{2}} \frac{\pi}{\eta}\left(\frac{r_{\mathrm{R}}}{C_{\mathrm{R}} r}\right)^{3 / 2}\right]$,

or equivalently (cf. Eq. (13) of Daisaka et al. 2001)

$\tau_{\perp} \gtrsim \max \left(0.08 r_{\mathrm{H}}^{*-3}, 0.2 r_{\mathrm{H}}^{*-3 / 2}\right)$,

with the transition between the two regimes at $r_{\mathrm{H}}^{*}=6^{-1 / 3} \approx 0.55$. Here, the auxiliary variable $r_{\mathrm{H}}^{*}$ (closely related to the Roche limit) is defined as the ratio of the mutual Hill radius of two disc particles $r_{\mathrm{H}}=\left[2 m /\left(3 M_{\star}\right)\right]^{1 / 3} r$ to the sum of their physical radii:

$$
\begin{aligned}
r_{\mathrm{H}}^{*}=\frac{r_{\mathrm{H}}}{2 s} & =\left(\frac{\pi}{9} \frac{\rho_{\mathrm{d}}}{M_{\star}}\right)^{1 / 3} r \approx 0.7\left(\frac{M_{\star}}{0.6 \mathrm{M}_{\odot}}\right)^{-1 / 3}\left(\frac{\rho_{\mathrm{d}}}{3 \mathrm{~g} \mathrm{~cm}^{-3}}\right)^{1 / 3}\left(\frac{r}{1 \mathrm{R}_{\odot}}\right) \\
& =\frac{C_{\mathrm{R}}}{12^{1 / 3}} \frac{r}{r_{\mathrm{R}}} \approx 0.87\left(\frac{C_{\mathrm{R}}}{2.0}\right)\left(\frac{r / r_{\mathrm{R}}}{1}\right) .
\end{aligned}
$$

Figure 2 shows the gravitational stability criterion. Comparing the typical vertical optical depth of the discs we consider in this study $\left(\tau_{\perp} \gtrsim 10^{2}\right.$; see Eq. (4)) to this criterion demonstrates that the discs are clearly gravitationally unstable. In fact, gravitational instabilities will occur in any vertically optically thick disc located in the outer half of the tidal disruption zone. In such discs, the interplay between gravitational instability, tidal forces, and Keplerian shear will lead to the continuous creation and destruction of transient density enhancements known as self-gravity 
wakes (Salo 1992; see also Julian \& Toomre 1966). The wakes are typically spaced by distances of around $\lambda_{\mathrm{T}}$ and their presence has been demonstrated observationally for Saturn's dense A and B rings (Colwell et al. 2009). In a gravitationally unstable disc, the radial velocity dispersion no longer follows Eq. (9) but instead adjusts to a higher value such that $Q_{\mathrm{T}} \simeq 2$ (Salo 1995, 2012). Importantly, the self-gravity wakes strongly affect the disc's effective viscosity: the wake structures lead to systematic motions of the disc particles and they exert gravitational torques, thereby enhancing the angularmomentum transport in the disc (Daisaka et al. 2001).

\subsection{Disc evolution}

Two processes are likely to be important for the evolution of massive debris discs around WDs: viscous spreading (see, e.g., Pringle 1981) and Poynting-Robertson (PR) drag (see, e.g., Burns et al. 1979). The former is known to govern the long-term evolution of massive planetary rings (Salmon et al. 2010), while the latter is key in the evolution of (asteroid-mass) WD debris discs (Rafikov 2011b; Bochkarev \& Rafikov 2011). In this section, we review how both processes operate and determine which of the two dominates under what conditions. A more rigorous analysis of their interplay using a torque-density balance is presented in Appendix A.

\subsubsection{Viscous evolution}

Viscous spreading of an astrophysical disc transports angular momentum outwards, along with some of the disc's mass, while moving most of the mass inwards. The disc's viscosity depends on whether it is gravitationally stable. As determined in Sect. 2.2, the discs considered in this study are gravitationally unstable and exhibit self-gravity wakes that enhance the effective viscosity. Dimensional analysis can be used to show that the effective kinetic viscosity of a gravitationally unstable (fluid) disc should be of the form $v \sim \lambda_{\mathrm{T}}^{2} \Omega_{\mathrm{K}} \sim G^{2} \Sigma^{2} / \Omega_{\mathrm{K}}^{3}$ (e.g., Lin \& Pringle 1987). For particle discs, Daisaka et al. (2001; see also Yasui et al. 2012) find

$v \simeq C_{\mathrm{visc}}\left(r_{\mathrm{H}}^{*}\right) \frac{G^{2} \Sigma^{2}}{\Omega_{\mathrm{K}}^{3}}, \quad C_{\mathrm{visc}}\left(r_{\mathrm{H}}^{*}\right) \simeq 26 r_{\mathrm{H}}^{* 5}$.

Here, $C_{\text {visc }}$ is an empirical correction factor, determined from a fit to viscosities derived from numerical simulations, with values $0.41 \lesssim$ $C_{\text {vise }} \lesssim 13$ in the range $0.5 \leq r / r_{\mathrm{R}} \leq 1$ (assuming $C_{\mathrm{R}}=2.0$ ). The strong additional radial dependence in $C_{\text {visc }}$ is thought to be a result of the incompressibility of the particles that make up the disc.

The evolution of discs whose viscosity is given by a double power-law function of radius and surface density (i.e., $v \propto \Sigma^{\alpha} r^{\beta}$ ) can usually be described analytically in terms of similarity solutions (Pringle 1991). Unfortunately, the power-law exponents in the present case ( $\alpha=2, \beta=19 / 2)$ fall outside the regime in which these known solutions are valid. The approximate evolution of the disc, however, can still be understood by considering its viscous timescale, i.e., the characteristic timescale on which viscous spreading causes changes in the local surface density of the disc. For an initially narrow disc at radius $r$, the viscous timescale is given by (e.g., Pringle 1981; Hahn 2009)

$t_{\mathrm{visc}} \sim \frac{r^{2}}{12 v} \simeq \frac{3^{7 / 3}}{104 \pi^{5 / 3}} \frac{1}{\sqrt{G}} \frac{M_{\star}^{19 / 6}}{\rho_{\mathrm{d}}^{5 / 3}} \frac{1}{\Sigma^{2} r^{15 / 2}}$.

Because the effective viscosity caused by gravitational instabilities increases rapidly with distance, the outer parts of the disc evolve much faster than the inner parts. For an initially narrow disc, the outer edge will therefore move out faster than the inner edge moves in. To conserve angular momentum, however, most of the disc's mass moves inwards. The combined effect of these different factors is that the disc develops a pile-up of material close to its inner edge. After some time, when the disc's initial radial distribution has faded, the surface density will roughly follow a profile of equal viscous timescale, with a radial dependence $\Sigma_{\text {visc }} \propto r^{-15 / 4}$.

\subsubsection{Poynting-Robertson-drag disc evolution}

Stellar radiation that hits the disc exerts PR drag on the disc and as a result disc material will spiral inwards towards the WD. For a disc that is optically thick to the impinging stellar radiation $\left(\tau_{\|} \gg 1\right)$, the PR-drag-induced mass flow is given by (Rafikov 2011b)

$\dot{M}_{\mathrm{PR}}(r) \simeq \zeta \frac{L_{\star}}{c^{2}} \simeq \frac{16}{3} \frac{\sigma_{\mathrm{SB}}}{c^{2}} \frac{T_{\star}^{4} R_{\star}^{3}}{r}$.

Here, $c$ is the speed of light, $L_{\star}=4 \pi R_{\star}^{2} \sigma_{\mathrm{SB}} T_{\star}^{4}$ is the WD's luminosity, $\sigma_{\mathrm{SB}}$ is the Stefan-Boltzmann constant, and $T_{\star}$ is the WD's effective temperature. Note that this mass flow (for an optically thick disc) is independent of any disc properties such as surface density $\Sigma$ or grain size $s$.

The global evolution of PR-drag-dominated WD debris discs was studied in further detail by Bochkarev \& Rafikov (2011). They find that the typical timescale for the evolution of optically thick discs is given $b^{2}$

$t_{\mathrm{PR}} \sim \frac{2 \pi \Sigma r^{2}}{\dot{M}_{\mathrm{PR}}} \simeq \frac{3 \pi}{8} \frac{c^{2}}{\sigma_{\mathrm{SB}}} \frac{1}{T_{\star}^{4} R_{\star}^{3}} \Sigma r^{3}$.

Specifically for narrow discs located in outer parts of the tidal disruption zone (i.e., the type of discs we focus on here), Bochkarev \& Rafikov (2011, see their Sect. 4.1.1) find the following behaviour: (1) surface densities in the bulk of the disc gradually go down; (2) the disc develops a sharp outer edge that moves inwards with time; and (3) an optically thin $\left(\tau_{\|} \lesssim 1\right)$ tail appears, extending inwards from the inner edge of the optically thick region, through which the disc material drains into the sublimation zone.

\subsubsection{Viscous spreading vs. Poynting-Robertson drag}

The scaling of the viscous and PR-drag disc-evolution timescales with surface density and radius $\left(t_{\text {visc }} \propto \Sigma^{-2} r^{-15 / 2} ; t_{\mathrm{PR}} \propto \Sigma r^{3}\right)$ shows that viscous spreading plays a larger role in the evolution of dense discs at large distances, while PR drag is more important close to the star and for lower surface densities (see Fig. 3). By setting the two timescales equal to each other $\left(t_{\mathrm{visc}}=t_{\mathrm{PR}}\right)$ and solving for $\Sigma$, we find the critical surface mass density $\sum_{\text {crit }}$ above which viscous spreading dominates the disc's evolution rather than PR drag (dashed white line in Fig. 3, left-hand panel)

$\Sigma_{\text {crit }} \simeq \frac{3^{4 / 9}}{13^{1 / 3} \pi^{8 / 9}} \frac{\sigma_{\mathrm{SB}}^{1 / 3}}{c^{2 / 3} G^{1 / 6}} \frac{T_{\star}^{4 / 3} M_{\star}^{19 / 18} R_{\star}}{\rho_{\mathrm{d}}^{5 / 9}} \frac{1}{r^{7 / 2}}$.

For an assumed value for the disc-width parameter $\delta$, this critical surface density translates (via Eq. (3)) into a critical disc mass

2 This 'PR-drag disc-evolution timescale' is not to be confused with the more commonly used 'PR-drag timescale', i.e., the time it takes for an individual particle to migrate from some radius onto its host star. These two are only the same (to within a factor of order unity) for optically thin discs (Bochkarev \& Rafikov 2011). 

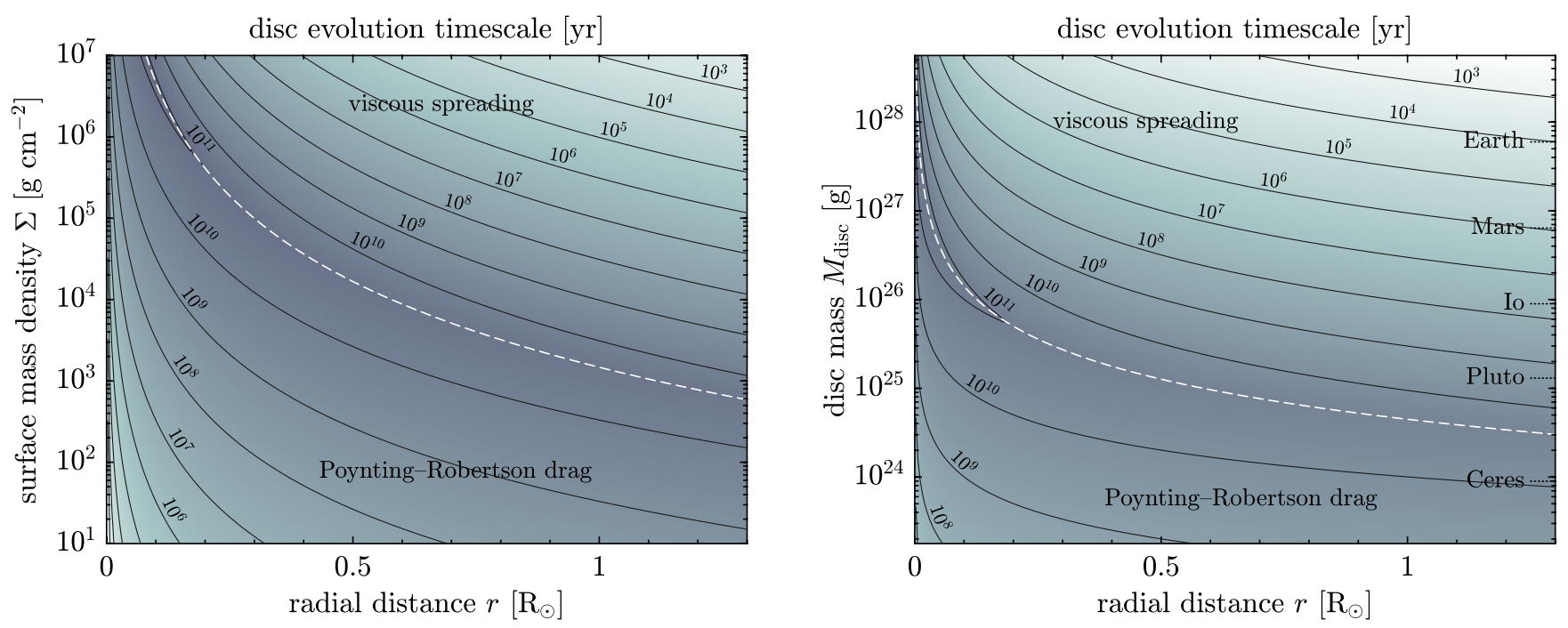

Figure 3. Contours of equal disc evolution timescale $t_{\mathrm{evol}}=\min \left(t_{\mathrm{visc}}, t_{\mathrm{PR}}\right)$ as a function of radial distance $r$ and surface mass density $\Sigma($ left-hand panel) or disc mass $M_{\text {disc }}$ (right-hand panel). The dashed white lines indicate conditions for which the two timescales are equal (corresponding to Eqs. (17) and (18)). We have assumed stellar parameters $T_{\star}=10,000 \mathrm{~K}, M_{\star}=0.6 \mathrm{M}_{\odot}, R_{\star}=0.0125 \mathrm{R}_{\odot}$, and a material density of $\rho_{\mathrm{d}}=3 \mathrm{~g} \mathrm{~cm}{ }^{-3}$. Disc masses were computed from surface densities using a disc-width parameter of $\delta=0.1$. For reference, the masses of several Solar-System objects are indicated.

separating viscous-spreading and PR-drag dominated discs (dashed white line in Fig. 3, right-hand panel)

$$
\begin{aligned}
M_{\text {crit }} \simeq & \frac{2 \times 3^{4 / 9} \pi^{1 / 9}}{13^{1 / 3}} \frac{\sigma_{\mathrm{SB}}^{1 / 3}}{c^{2 / 3} G^{1 / 6}} \frac{T_{\star}^{4 / 3} M_{\star}^{19 / 18} R_{\star}}{\rho_{\mathrm{d}}^{5 / 9}} \frac{\delta}{r^{3 / 2}} \\
\approx & 4.5 \times 10^{24} \mathrm{~g}\left(\frac{T_{\star}}{10,000 \mathrm{~K}}\right)^{4 / 3}\left(\frac{M_{\star}}{0.6 \mathrm{M}_{\odot}}\right)^{19 / 18}\left(\frac{R_{\star}}{0.0125 \mathrm{R}_{\odot}}\right) \\
& \times\left(\frac{\rho_{\mathrm{d}}}{3 \mathrm{~g} \mathrm{~cm}^{-3}}\right)^{-5 / 9}\left(\frac{\delta}{0.1}\right)\left(\frac{r}{1 \mathrm{R}_{\odot}}\right)^{-3 / 2} .
\end{aligned}
$$

Noting that the timescale prescriptions used to derive Eq. (17) are order-of-magnitude estimates, we rederive $\Sigma_{\text {crit }}$ in Appendix A using a torque-density balance between viscous spreading and PR drag. The expression for $\Sigma_{\text {crit }}$ found from this exercise is the same as Eq. (17) except for a small difference in the numerical coefficient, which is a factor $(5 / 2)^{1 / 3} \approx 1.36$ lower in the torque-densitybalance result. For reasons of internal consistency, we continue our analysis using Eq. (17) as prescription for $\Sigma_{\text {crit }}$.

We stress that the boundary between viscous and PR-drag disc evolution is independent of grain size. This is the case as long as the particles are small enough to ensure that the disc is gravitationally unstable, so that using Eq. (13) for the effective viscosity is valid. Comparing the typical values of $\Sigma_{\text {crit }}$ (Fig. 3) with the gravitational-stability criterion (Fig. 2) shows that a marginally viscous-spreading-dominated disc will be gravitationally unstable for particle sizes of $s \lesssim 1 \mathrm{~m}$ (through Eq. (4)). For more massive discs this maximum grain size goes up, making the validity requirement less stringent. Furthermore, Fig. 2 shows that any disc that is gravitationally unstable is also optically thick to the stellar irradiation $\left(\tau_{\|} \gg 1\right)$, so our treatment of PR drag in the derivation of $\Sigma_{\text {crit }}$ is justified as well.

\subsubsection{Planet-producing discs}

Discs whose initial mass is substantially above the critical level $M_{\text {crit }}$ may viscously spread to beyond the Roche limit, where disc material can coagulate to ultimately form a new generation of (mi- nor) planets (see Sect. 2.4). More specifically, there is a disc mass $M_{\text {form }}$ above which an initially narrow disc located at radius $r_{0}$ will radially spread enough for some of its mass to end up beyond the Roche limit. We now proceed to estimate this minimum disc mass for planet formation.

An analytical approximation to $M_{\text {form }}$ can be derived by assuming that half of the disc's initial mass spreads outwards from $r_{0}$ and that this material follows a radial surface-density profile $\Sigma_{\mathrm{visc}} \propto$ $r^{-15 / 4}$ (appropriate for viscously spreading discs; see Sect. 2.3.1). For the disc's outer edge to reach the Roche limit, the outwardsspreading material needs to maintain a surface density above the critical level $\Sigma_{\text {crit }}$ at all distances between the initial radius and the Roche limit (otherwise PR drag will halt further outward spreading). Because $\Sigma_{\text {visc }}$ decreases (slightly) faster with radius than $\Sigma_{\text {crit }}$, one only needs to assess this requirement at the most distant point, the Roche limit. The least massive disc to reach the Roche limit will thus have a surface-density profile that has a radial dependence of $\Sigma_{\text {visc }} \propto r^{-15 / 4}$ and passes through the point $\left\{r_{\mathrm{R}}, \Sigma_{\text {crit }}\left(r_{\mathrm{R}}\right)\right\}$. The profile that meets these two conditions is given by

$\sum_{\text {visc }}\left[t_{\text {crit }}\left(r_{\mathrm{R}}\right)\right] \simeq \frac{3^{19 / 36} C_{\mathrm{R}}^{1 / 4}}{2^{1 / 6} 13^{1 / 3} \pi^{35 / 36}} \frac{\sigma_{\mathrm{SB}}^{1 / 3}}{c^{2 / 3} G^{1 / 6}} \frac{T_{\star}^{4 / 3} M_{\star}^{41 / 36} R_{\star}}{\rho_{\mathrm{d}}^{23 / 36}} \frac{1}{r^{15 / 4}}$,

where $t_{\text {crit }}$ is to be defined later (see Sect. 2.3.5). ${ }^{3}$

Following our assumption that half the disc's mass spreads outwards, a viscously spreading disc will reach the Roche limit if half its mass $M_{\text {disc }} / 2$ exceeds the mass contained between $r_{0}$ and $r_{\mathrm{R}}$ in the surface-density distribution given by Eq. (19). Thus, the
3 Equation (19) can be derived from Eq. (14) by setting $t_{\text {visc }}=t_{\text {crit }}\left(r_{\mathrm{R}}\right)$, as
given by Eq. (22), and solving for $\Sigma$. 


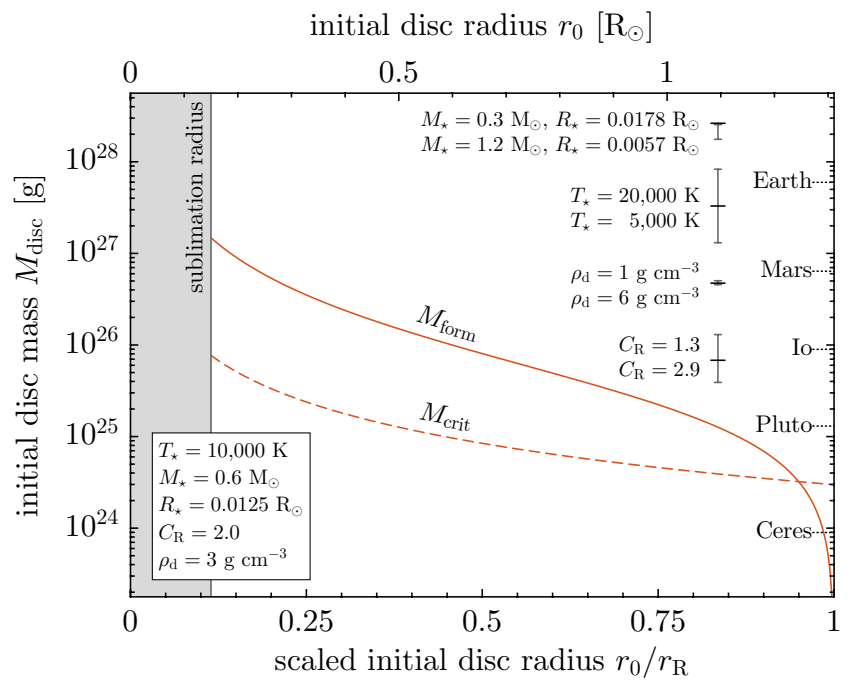

Figure 4. Minimum disc mass for planet formation $M_{\text {form }}$ (above which viscosity spreads an initially narrow disc to beyond the Roche limit; Eq. (20); solid line) as a function of the disc's initial radius $r_{0}$ scaled to the Roche limit $r_{\mathrm{R}}$. Also shown is the critical disc mass $M_{\text {crit }}$ (above which viscous spreading dominates the disc's evolution over PR drag; Eq. (18); dashed line) for a disc-width parameter of $\delta=0.1$. Both curves are computed using the fiducial parameter values listed in the bottom-left corner (box). Error bars in the top right indicate how $M_{\text {form }}$ would change relative to the fiducial level (dark horizontal bars) by varying the stellar mass $M_{\star}$, stellar temperature $T_{\star}$, material density $\rho_{\mathrm{d}}$, or Roche prefactor $C_{\mathrm{R}}$ within reasonable ranges. We used the WD mass-radius relation to change the stellar radius $R_{\star}$ along with $M_{\star}$. Note that the absolute-distance values given along the top axis change if $M_{\star}, \rho_{\mathrm{d}}$, or $C_{\mathrm{R}}$ is altered from its fiducial value.

minimum disc mass for planet formation can be calculated as

$$
\begin{aligned}
M_{\text {form }} \simeq & \int_{r_{0}}^{r_{\mathrm{R}}} 4 \pi r \sum_{\text {visc }}\left[t_{\text {crit }}\left(r_{\mathrm{R}}\right)\right] \mathrm{d} r \\
\simeq & \frac{32 \pi^{11 / 18}}{7 \times 3^{1 / 18} 13^{1 / 3} C_{\mathrm{R}}^{3 / 2}} \frac{\sigma_{\mathrm{SB}}^{1 / 3}}{c^{2 / 3} G^{1 / 6}} \frac{T_{\star}^{4 / 3} M_{\star}^{5 / 9} R_{\star}}{\rho_{\mathrm{d}}^{1 / 18}}\left[\left(\frac{r_{\mathrm{R}}}{r_{0}}\right)^{7 / 4}-1\right] \\
\approx & 3.5 \times 10^{25} \mathrm{~g}\left(\frac{T_{\star}}{10,000 \mathrm{~K}}\right)^{4 / 3}\left(\frac{M_{\star}}{0.6 \mathrm{M}_{\odot}}\right)^{5 / 9}\left(\frac{R_{\star}}{0.0125 \mathrm{R}_{\odot}}\right) \\
& \times\left(\frac{C_{\mathrm{R}}}{2.0}\right)^{-3 / 2}\left(\frac{\rho_{\mathrm{d}}}{3 \mathrm{~g} \mathrm{~cm}^{-3}}\right)^{-1 / 18}\left[\left(\frac{r_{\mathrm{R}}}{r_{0}}\right)^{7 / 4}-1\right] .
\end{aligned}
$$

The distance-dependent factor in this equation (in square brackets) equals unity at $r_{0} / r_{\mathrm{R}}=2^{-4 / 7} \approx 0.67$ and goes to zero for $r_{0} / r_{\mathrm{R}} \rightarrow 1$, owing to the fact that a disc that is initially already very close to the Roche limit need not be very massive to spread beyond it.

Figure 4 shows the minimum disc mass for planet formation as a function of the disc's initial radius $r_{0}$. This demonstrates that massive debris discs located in the outer parts of the tidal disruption zone (i.e., Io-mass discs with $r_{0} / r_{\mathrm{R}} \gtrsim 0.5$ ) will spread out to beyond the Roche limit. ${ }^{4}$ How $M_{\text {form }}$ is affected by the uncertainties in various parameters is illustrated by the error bars in the figure. When

\footnotetext{
4 For initial radii very close to the Roche limit, much less massive discs can also reach the Roche limit, and in Fig. 4 the $M_{\text {form }}$ curve even drops below the $M_{\text {crit }}$ one. This is because a well-defined $M_{\text {crit }}$ requires a non-zero scaled disc width $\delta$, while the derivation of $M_{\text {form }}$ assumes that the disc is initially infinitely thin.
}

the WD mass-radius relation is taken into account, the stellar-mass and radius dependencies roughly cancel each other, except for the most massive WDs. Specifically, using Eq. (2.4) of Veras (2016; originally from Nauenberg 1972) as mass-radius relation, the combined effect of $M_{\star}$ and $R_{\star}$ on $M_{\text {form }}$ varies by less than $10 \%$ for WD masses in the range $0.1 \mathrm{M}_{\odot} \lesssim M_{\star} \lesssim 1 \mathrm{M}_{\odot}$.

\subsubsection{Disc lifetimes}

The lifetime of a disc $t_{\text {disc }}$ is defined as the time after which all the material that originally resides in the disc has either flowed beyond the Roche limit or disappeared inside the sublimation radius. For PR-drag-dominated discs, the evolution timescale decreases as material is removed. Their lifetime is therefore dominated by the initial stages of disc evolution, and can be approximated by (cf. Eq. (49) of Metzger et al. 2012)

$$
\begin{aligned}
t_{\text {disc }}\left(M_{\text {disc }} \ll M_{\text {crit }}\right) \simeq & \frac{M_{\text {disc }}}{\dot{M}_{\mathrm{PR}}\left(r_{0}\right)} \simeq \frac{3}{16} \frac{c^{2}}{\sigma_{\mathrm{SB}}} \frac{M_{\mathrm{disc}} r_{0}}{T_{\star}^{4} R_{\star}^{3}} \\
\approx & 1.0 \mathrm{Gyr}\left(\frac{T_{\star}}{10,000 \mathrm{~K}}\right)^{-4}\left(\frac{R_{\star}}{0.0125 \mathrm{R}_{\odot}}\right)^{-3} \\
& \times\left(\frac{M_{\mathrm{disc}}}{10^{24} \mathrm{~g}}\right)\left(\frac{r_{0}}{1 \mathrm{R}_{\odot}}\right) .
\end{aligned}
$$

This is equivalent to the PR-drag disc-evolution timescale $t_{\mathrm{PR}}$ for the initial state of the disc multiplied by a factor $\delta$ (see Eq. (16)).

For discs dominated by viscous spreading, the evolution timescale instead increases as the surface density goes down. A viscosity-dominated disc will spread out slower and slower, until its surface density reaches the critical surface density $\Sigma_{\text {crit }}$. Once this happens, PR drag will take over the evolution of the disc's outer parts, developing a sharp outer edge that moves inwards with time (Bochkarev \& Rafikov 2011). The disc's evolution timescale when the transition occurs $t_{\text {crit }}$ can be found from Eq. (14) or (16) with $\Sigma=\Sigma_{\text {crit }}$, which both give

$t_{\text {crit }} \simeq \frac{3^{13 / 9} \pi^{1 / 9}}{8 \times 13^{1 / 3}} \frac{c^{4 / 3}}{\sigma_{\mathrm{SB}}^{2 / 3} G^{1 / 6}} \frac{M_{\star}^{19 / 18}}{T_{\star}^{8 / 3} R_{\star}^{2} \rho_{\mathrm{d}}^{5 / 9}} \frac{1}{\sqrt{r}}$.

Of particular interest is the value of this timescale at the Roche limit $t_{\text {crit }}\left(r_{\mathrm{R}}\right)$. Since Eq. (22) is a decreasing function of $r$, and the disc is confined to $r<r_{\mathrm{R}}$, this is the lowest possible value of $t_{\text {crit }}$.

Around the time of the transition from viscosity-dominated to PR-drag-dominated disc evolution, a disc that is massive enough to reach the Roche limit will undergo viscous evolution for a timespan of about $t_{\text {crit }}\left(r_{\mathrm{R}}\right)$, followed by PR-drag evolution for another leg of time of roughly the same duration. The total lifetime of such discs is dominated by the duration of this episode around the transition. It is independent of disc mass and can be approximated by

$$
\begin{aligned}
t_{\text {disc }}\left(M_{\text {disc }} \gg M_{\text {form }}\right) \simeq & 2 t_{\text {crit }}\left(r_{\mathrm{R}}\right) \\
\simeq & \frac{3^{23 / 18} \pi^{5 / 18}}{2^{5 / 3} 13^{1 / 3} \sqrt{C_{\mathrm{R}}}} \frac{c^{4 / 3}}{\sigma_{\mathrm{SB}}^{2 / 3} G^{1 / 6}} \frac{M_{\star}^{8 / 9}}{T_{\star}^{8 / 3} R_{\star}^{2} \rho_{\mathrm{d}}^{7 / 18}} \\
\approx & 78 \mathrm{Gyr}\left(\frac{C_{\mathrm{R}}}{2.0}\right)^{-1 / 2}\left(\frac{T_{\star}}{10,000 \mathrm{~K}}\right)^{-8 / 3}\left(\frac{M_{\star}}{0.6 \mathrm{M}_{\odot}}\right)^{8 / 9} \\
& \times\left(\frac{R_{\star}}{0.0125 \mathrm{R}_{\odot}}\right)^{-2}\left(\frac{\rho_{\mathrm{d}}}{3 \mathrm{~g} \mathrm{~cm}^{-3}}\right)^{-5 / 9}
\end{aligned}
$$

This shows that massive WD debris discs can survive against viscous spreading and PR drag for longer than the age of the universe. They may, of course, still be destroyed by mechanisms not accounted for in our analysis (see Sect. 4.2). 


\subsection{Second-generation (minor) planets}

When material from a massive $\operatorname{disc}\left(M_{\text {disc }} \gtrsim M_{\text {form }}\right)$ flows beyond the Roche limit, its constituent particles are no longer prevented from coagulating by tidal forces and can stick together in gravitationally bound aggregates. These aggregates can then merge to form larger bodies, eventually producing a set of second-generation (minor) planets. In this section, we investigate these processes and infer what the basic properties of the newly formed bodies may be.

\subsubsection{Disc properties at the Roche limit}

The planet-formation process depends directly on the state of the disc at its outer edge - i.e., at the Roche limit. Specifically, the properties of the newly formed (minor) planets are determined by the outward mass flow rate through the Roche limit $\dot{M}_{\text {out }}$, which, in turn, is controlled by the disc's local surface density. For this reason, we often express formulae in this section in terms of the disc's Roche-limit surface density $\Sigma_{\mathrm{R}} \equiv \Sigma\left(r_{\mathrm{R}}\right)$. A rough estimate of this quantity at the time when planet formation starts $t_{\text {start }}$ can be obtained by assuming that half the disc's mass spreads out between $r_{0}$ and $r_{\mathrm{R}}$, following the viscous-spreading-dictated surface-density profile $\Sigma_{\text {visc }} \propto r^{-15 / 4}$ (see Sect. 2.3.1). This gives

$$
\begin{aligned}
\Sigma_{\mathrm{R}}\left(t_{\text {start }}\right) \simeq & \frac{7}{16} \frac{M_{\text {disc }}}{\pi r_{\mathrm{R}}^{2}}\left[\left(\frac{r_{\mathrm{R}}}{r_{0}}\right)^{7 / 4}-1\right]^{-1} \\
\approx & 1.7 \times 10^{3} \mathrm{~g} \mathrm{~cm}^{-2}\left(\frac{C_{\mathrm{R}}}{2.0}\right)\left(\frac{M_{\star}}{0.6 \mathrm{M}_{\odot}}\right)^{1 / 3}\left(\frac{\rho_{\mathrm{d}}}{3 \mathrm{~g} \mathrm{~cm}^{-3}}\right)^{-1 / 3} \\
& \times\left(\frac{M_{\text {disc }}}{10^{26} \mathrm{~g}}\right)\left[\left(\frac{r_{\mathrm{R}}}{r_{0}}\right)^{7 / 4}-1\right]^{-1} .
\end{aligned}
$$

Over time, the Roche-limit surface density will slowly go down. When it approaches the local critical level $\Sigma_{\text {crit }}\left(r_{\mathrm{R}}\right)$, the outward mass flow through the Roche limit is gradually quenched by PR drag, eventually terminating the production of new planets. Therefore, an approximate lower limit on the value of $\Sigma_{\mathrm{R}}$ relevant to second-generation planet formation is (see Fig. 3, left-hand panel)

$$
\begin{aligned}
\Sigma_{\text {crit }}\left(r_{\mathrm{R}}\right) \simeq & \frac{2^{7 / 3} \pi^{5 / 18}}{3^{13 / 18} 13^{1 / 3} C_{\mathrm{R}}^{7 / 2}} \frac{\sigma_{\mathrm{SB}}^{1 / 3}}{c^{2 / 3} G^{1 / 6}} \frac{T_{\star}^{4 / 3} R_{\star} \rho_{\mathrm{d}}^{11 / 18}}{M_{\star}^{1 / 9}} \\
\approx & 570 \mathrm{~g} \mathrm{~cm}^{-2}\left(\frac{T_{\star}}{10,000 \mathrm{~K}}\right)^{4 / 3}\left(\frac{M_{\star}}{0.6 \mathrm{M}_{\odot}}\right)^{-1 / 9}\left(\frac{R_{\star}}{0.0125 \mathrm{R}_{\odot}}\right) \\
& \times\left(\frac{C_{\mathrm{R}}}{2.0}\right)^{-7 / 2}\left(\frac{\rho_{\mathrm{d}}}{3 \mathrm{~g} \mathrm{~cm}^{-3}}\right)^{11 / 18} .
\end{aligned}
$$

From Eqs. (24) and (25) we find that the value of $\Sigma_{\mathrm{R}}$ typically lies in the range $10^{2} \mathrm{~g} \mathrm{~cm}^{-2} \lesssim \Sigma_{\mathrm{R}} \lesssim 10^{5} \mathrm{~g} \mathrm{~cm}^{-2}$ for the discs we consider while they are overflowing the Roche limit.

Given that changes in the disc's local surface density happen on the local viscous timescale, the disc's mass outflow rate at the Roche limit can be approximated by (cf. Eq. (S4) of CC12)

$$
\begin{aligned}
\dot{M}_{\text {out }} & \simeq \frac{\pi r_{\mathrm{R}}^{2} \Sigma_{\mathrm{R}}}{t_{\mathrm{visc}}\left(\Sigma_{\mathrm{R}}, r_{\mathrm{R}}\right)} \simeq \frac{3^{5 / 6} 13 C_{\mathrm{R}}^{19 / 2}}{2^{10 / 3} \sqrt{\pi}} \sqrt{G} \frac{\Sigma_{\mathrm{R}}^{3}}{\rho_{\mathrm{d}}^{3 / 2}} \\
& \approx 6.5 \times 10^{7} \mathrm{~g} \mathrm{~s}^{-1}\left(\frac{C_{\mathrm{R}}}{2.0}\right)^{19 / 2}\left(\frac{\rho_{\mathrm{d}}}{3 \mathrm{~g} \mathrm{~cm}^{-3}}\right)^{-3 / 2}\left(\frac{\Sigma_{\mathrm{R}}}{10^{3} \mathrm{~g} \mathrm{~cm}^{-2}}\right)^{3} .
\end{aligned}
$$

As expected, for a Roche-limit surface mass density at the critical level (i.e., $\Sigma_{\mathrm{R}}=\Sigma_{\text {crit }}\left(r_{\mathrm{R}}\right)$ ), the outward mass flow rate due to viscous spreading roughly matches the inward mass flow rate from PR drag: $\dot{M}_{\text {out }}\left[\sum_{\text {crit }}\left(r_{\mathrm{R}}\right)\right] \simeq \dot{M}_{\mathrm{PR}}\left(r_{\mathrm{R}}\right) / 2$. The discrepancy of a factor 2 can be explained by the approximate nature of Eqs. (17) and (26).

\subsubsection{Planetesimal formation through gravitational instabilities}

As the disc expands beyond the Roche limit, gravitational instabilities cause portions of the material beyond $r_{\mathrm{R}}$ to collapse into gravitationally bound aggregates. Since the instabilities typically have sizes around Toomre's critical wavelength $\lambda_{\mathrm{T}}$ (see Eq. (8)), these newly formed, rubble-pile-type planetesimals will have masses of the order of the local Toomre mass $M_{\mathrm{T}}$, defined as the mass enclosed in a circular portion of the disc with diameter $\lambda_{\mathrm{T}}$ (cf. Goldreich \& Ward 1973; Nelson 2006; Michikoshi et al. 2007, 2009). ${ }^{5}$ At the Roche limit, the Toomre mass is

$$
\begin{aligned}
M_{\mathrm{T}}\left(r_{\mathrm{R}}\right) & =\pi\left[\frac{\lambda_{\mathrm{T}}\left(r_{\mathrm{R}}\right)}{2}\right]^{2} \Sigma_{\mathrm{R}}=\frac{9 \pi^{3} C_{\mathrm{R}}^{6}}{4} \frac{\Sigma_{\mathrm{R}}^{3}}{\rho_{\mathrm{d}}^{2}} \\
& \approx 5.0 \times 10^{11} \mathrm{~g}\left(\frac{C_{\mathrm{R}}}{2.0}\right)^{6}\left(\frac{\rho_{\mathrm{d}}}{3 \mathrm{~g} \mathrm{~cm}^{-3}}\right)^{-2}\left(\frac{\Sigma_{\mathrm{R}}}{10^{3} \mathrm{~g} \mathrm{~cm}^{-2}}\right)^{3} .
\end{aligned}
$$

The typical value of $5.0 \times 10^{11} \mathrm{~g}$ corresponds to a body with a radius of about $34 \mathrm{~m}$, assuming its density is the same as the disc material. As $\Sigma_{\mathrm{R}}$ goes down with time, the mass of the planetesimals decreases. Since the disc's mass outflow rate $\dot{M}_{\text {out }}$ goes down at the same rate, however, Toomre-mass bodies are created at a constant rate, namely one planetesimal per time interval

$t_{\mathrm{T}}=\frac{M_{\mathrm{T}}\left(r_{\mathrm{R}}\right)}{\dot{M}_{\text {out }}} \simeq \frac{2^{4 / 3} 3^{2 / 3} \pi^{3}}{13 C_{\mathrm{R}}^{5}} P_{\mathrm{R}} \approx 2.1 \mathrm{~h}\left(\frac{C_{\mathrm{R}}}{2.0}\right)^{-7 / 2}\left(\frac{\rho_{\mathrm{d}}}{3 \mathrm{~g} \mathrm{~cm}^{-3}}\right)^{-1 / 2}$,

i.e., a few bodies per local orbital period.

The region just beyond the Roche limit may be populated with many co-orbital planetesimals with masses of around $M_{\mathrm{T}}\left(r_{\mathrm{R}}\right)$ each. What initially happens to these bodies will depend on their orbital evolution and the outcomes of mutual collisions. Here, we ignore the details of the initial coagulation process. Instead, we follow Charnoz et al. (2010) and CC12 in assuming that the planetesimals will accumulate into a single large body orbiting just beyond the Roche limit. Once this body is formed, it will absorb any additional material flowing out of the disc, as long as it is close enough to the Roche limit (see Sect. 2.4.4).

\subsubsection{Minor-planet growth and migration}

A body orbiting close to the disc's outer edge will grow by absorbing any disc material that flows beyond the Roche limit. At the same time, this body will experience torques from the disc associated with mean-motion resonances (MMRs), causing it to migrate outwards (e.g., Goldreich \& Tremaine 1980). The interaction between this simultaneous growth and outward migration was studied in detail by $\mathrm{CC} 12$, who developed a one-dimensional analytical model for the evolution of bodies formed by a viscously spreading particle disc that overflows the Roche limit. While their model was designed to study moons produced by planetary rings (see also Charnoz et al. 2010, 2011), it is formulated very generally, so we can readily apply many of its predictions to the present case of WD debris discs spawning second-generation (minor) planets.

Assuming a constant mass outflow rate from the disc's outer

5 Stricktly speaking, the relevant length scale is the wavelength of the fastest growing unstable mode $\lambda_{\mathrm{fgm}}=2 \sigma_{r} /(G \Sigma)$, rather than $\lambda_{\mathrm{T}}$, which is the wavelength of the longest instability. For $Q_{\mathrm{T}} \simeq 2$ (to which the gravitationally unstable disc material is expected to converge; see Sect. 2.2), however, these two length scales are approximately the same. 
edge at $r_{\mathrm{R}}$ (prescribed by Eq. (26)), CC12 find that the interplay of growth and outward migration leads to specific relations between a body's mass $M_{\mathrm{p}}$ and its separation from the disc's outer edge $r-r_{\mathrm{R}}$. These are best expressed in terms of the dimensionless quantities

$q \equiv \frac{M_{\mathrm{p}}}{M_{\star}}, \quad \Delta \equiv \frac{r-r_{\mathrm{R}}}{r_{\mathrm{R}}}$,

(i.e., the minor-planet mass ratio and the body's scaled distance from the Roche limit) and depend on just one free parameter: the disc's viscous evolution timescale at the Roche limit, scaled to the local orbital period ${ }^{6}$

$$
\begin{aligned}
\mathcal{T} & \equiv \frac{t_{\text {visc }}\left(\sum_{\mathrm{R}}, r_{\mathrm{R}}\right)}{P_{\mathrm{R}}} \simeq \frac{4 \pi^{1 / 3}}{3^{2 / 3} 13 C_{\mathrm{R}}^{9}} \frac{M_{\star}^{2 / 3} \rho_{\mathrm{d}}^{4 / 3}}{\sum_{\mathrm{R}}^{2}} \\
& \approx 2.1 \times 10^{13}\left(\frac{C_{\mathrm{R}}}{2.0}\right)^{-9}\left(\frac{M_{\star}}{0.6 \mathrm{M}_{\odot}}\right)^{2 / 3}\left(\frac{\rho_{\mathrm{d}}}{3 \mathrm{~g} \mathrm{~cm}^{-3}}\right)^{4 / 3}\left(\frac{\Sigma_{\mathrm{R}}}{10^{3} \mathrm{~g} \mathrm{~cm}^{-2}}\right)^{-2} .
\end{aligned}
$$

Given the values for $\Sigma_{\mathrm{R}}$ found in Sect. 2.4.1, we expect $\mathcal{T}$ to typically fall in the range $10^{9} \lesssim \mathcal{T} \lesssim 10^{15}$ for the discs we consider.

In the following two subsections, we review the model of $\mathrm{CC} 12$ and work out a few additional details. For reference, Fig. 5 already shows an overview of the resulting minor-planet massdistance relations for a few representative values of $\mathcal{T}$. These different $\mathcal{T}$-values can correspond to, e.g., the state of a given system at subsequent points in time, or to the states of systems with different initial disc masses and/or initial disc radii at the same point in time (see Sect. 2.4.1). In Fig. 5 and also in the rest of this paper, we assume that the mean density of the (minor) planets is the same as that of the disc material, so the radius of these bodies can be written as $R_{\mathrm{p}}=\left[3 M_{\mathrm{p}} /\left(4 \pi \rho_{\mathrm{d}}\right)\right]^{1 / 3}$.

\subsubsection{The continuous and discrete regimes}

In the initial stage of planet formation (the 'continuous regime'), a single growing body immediately absorbs any disc material that flows beyond the Roche limit. At the same time, this body moves away from the disc (through angular-momentum exchange via MMRs) at a rate that depends on its mass and distance to the disc's outer edge. As the body grows, its outward migration rate increases, but as it moves away from the disc, the migration rate rapidly decreases $\left(\mathrm{d} \Delta / \mathrm{d} t \propto q \Delta^{-3} ; \mathrm{CC} 12\right)$. The mass-distance relation for the continuous regime is found by integrating this migration rate under the assumption of a constant mass-absorption rate, which yields (CC12)

$q \simeq \frac{3^{3 / 2}}{2^{3} \sqrt{\mathcal{T}}} \Delta^{2}$.

When the growing, outwards-migrating body moves beyond about two Hill radii from the edge of the disc, it cannot absorb the disc material directly anymore, ending the continuous regime. This occurs at the scaled distance $\Delta_{\mathrm{c}}$ and mass ratio $q_{\mathrm{c}}$, given by (CC12)

$\Delta_{\mathrm{c}} \simeq \sqrt{\frac{3}{\mathcal{T}}} \approx \frac{1.73}{\sqrt{\mathcal{T}}}, \quad q_{\mathrm{c}} \simeq \frac{3^{5 / 2}}{2^{3 \mathcal{T}^{3 / 2}}} \approx \frac{1.95}{\mathcal{T}^{3 / 2}}$

6 When expressed in terms of absolute physical quantities rather than the dimensionless $\Delta, q$, and $\mathcal{T}$ (called $\tau_{\text {disk }}$ in the notation of CC12), there are small differences between our formulae and those of CC12. This is due to a difference in prescription for the viscous timescale ( $\mathrm{CC} 12$ omit the factor $1 / 12$ ) and a different treatment of the Roche prefactor (fixed to $C_{\mathrm{R}}=2.456$ by $\mathrm{CC} 12)$.

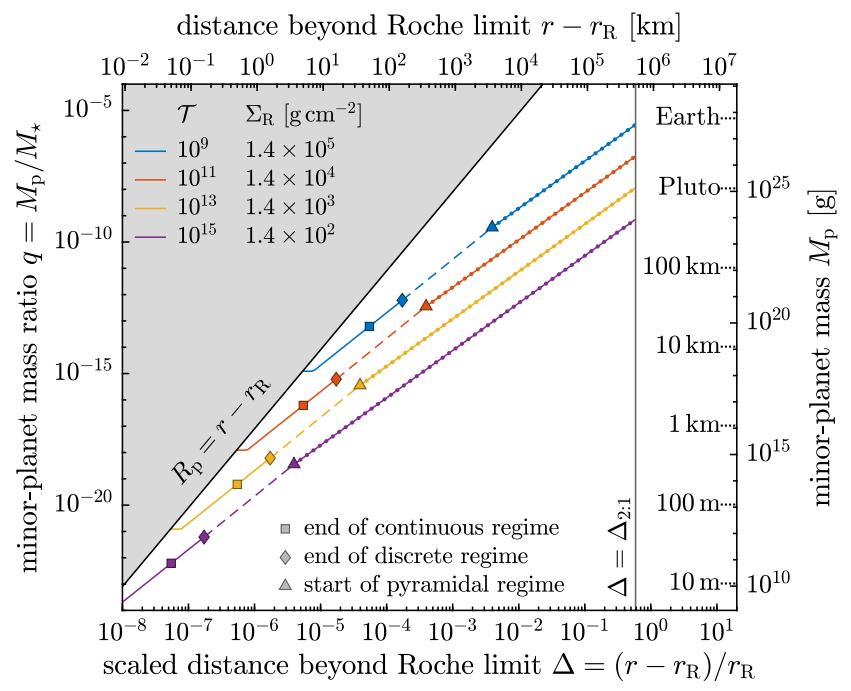

Figure 5. Minor-planet mass ratio $q$ as a function of the scaled distance beyond the Roche limit $\Delta$, for four different values of the dimensionless Roche-limit disc-evolution timescale $\mathcal{T}$. The mass-distance relations are composed of several parts: an absolute minimum set by the Roche-limit Toomre mass (Eq. (27); small horizontal line segments at low 4), Eq. (31) for the continuous and discrete regimes $\left(q \propto \Delta^{2}, \Delta<\Delta_{\mathrm{d}}\right)$, Eq. (36) for the pyramidal regime $\left(q \propto \Delta^{9 / 5}, \Delta>\Delta_{0}\right)$, and a hypothetical part connecting the discrete and pyramidal regimes (dashed lines). The knots in the pyramidal-regime line illustrate the average spacing of individual objects according to the number densities given by Eq. (37). Square, diamond, and triangle symbols mark the boundaries of the different regimes (Eqs. (32), (33), (38), and (39)). A vertical grey line shows $\Delta_{2: 1}$, the outermost scaled distance for which the migration rates underpinning the mass-distance relations are applicable. In terms of dimensional quantities, the Roche-limit surface mass densities $\Sigma_{\mathrm{R}}$ corresponding to the four lines are indicated in the legend, and absolute minor-planet masses and distances are given by the right-hand-side and top axes, respectively. For these dimensional versions (and for the Toomre masses), we use the stellar parameters $T_{\star}=10,000 \mathrm{~K}$, $M_{\star}=0.6 \mathrm{M}_{\odot}, R_{\star}=0.0125 \mathrm{R}_{\odot}$, a material density of $\rho_{\mathrm{d}}=3 \mathrm{~g} \mathrm{~cm}^{-3}$, and a Roche prefactor of $C_{\mathrm{R}}=2.0$. The top-left corner of parameter space (grey) is excluded, as this corresponds to bodies that are still in contact with the Roche limit (assuming their density equals that of the disc material).

Once a body migrates beyond $\Delta_{\mathrm{c}}$, the planet-formation process enters what is called the 'discrete regime'. Here, the further growth of the initial body happens via the transfer of small bodies that themselves accrete according to the continuous regime. On average, the mass-distance relation still follows Eq. (31), but the growth of the first-formed body will be discontinuous. The discrete regime ends when this object moves too far away to absorb additional newly formed continuous-regime bodies (two Hill radii from $\Delta_{\mathrm{c}}$ ). The scaled distance $\Delta_{\mathrm{d}}$ and mass-ratio $q_{\mathrm{d}}$ at this point are given by $(\mathrm{CC} 12)$

$\Delta_{\mathrm{d}} \simeq \frac{1}{Z_{0}^{3}} \sqrt{\frac{3}{\mathcal{T}}} \approx \frac{5.45}{\sqrt{\mathcal{T}}}, \quad q_{\mathrm{d}} \simeq \frac{3^{5 / 2}}{2^{3} Z_{0}^{6} \mathcal{T}^{3 / 2}} \approx \frac{19.3}{\mathcal{T}^{3 / 2}}$,

where $Z_{0} \approx 0.682$ is a numerical constant (specifically, the realvalued root of $Z^{3}+Z-1$ ).

Given that the dimensionless disc-evolution timescale $\mathcal{T}$ is very large for the WD debris discs we consider, the continuous and discrete regimes end very close to the Roche limit, and correspond to relatively small bodies (see Fig. 5). In a WD debris disc that is forming planets, mass continuously flows through the region between the Roche limit and $\Delta_{\mathrm{d}}$, transported initially by continuous- 
regime bodies and subsequently by discrete-regime bodies. While the mass-ratios of these bodies and the extents of the regimes all change with time as the disc's Roche-limit surface density $\Sigma_{\mathrm{R}}$ goes down, the rates at which bodies are created at $\left\{\Delta_{\mathrm{c}}, q_{\mathrm{c}}\right\}$ and $\left\{\Delta_{\mathrm{d}}, q_{\mathrm{d}}\right\}$ turn out to remain constant, independent of the properties of the disc. The corresponding time intervals between the formation of two subsequent bodies are given by (cf. CC12)

$t_{\mathrm{c}}=\frac{q_{\mathrm{c}} M_{\star}}{\dot{M}_{\text {out }}}=\frac{3^{13 / 6} \sqrt{13} C_{\mathrm{R}}^{5 / 2}}{2^{8 / 3} \sqrt{\pi}} P_{\mathrm{R}} \approx 4.4 \mathrm{~d}\left(\frac{C_{\mathrm{R}}}{2.0}\right)^{4}\left(\frac{\rho_{\mathrm{d}}}{3 \mathrm{~g} \mathrm{~cm}^{-3}}\right)^{-1 / 2}$,

for bodies with mass ratio $q_{\mathrm{c}}$ located at scaled distance $\Delta_{\mathrm{c}}$, and

$t_{\mathrm{d}}=\frac{q_{\mathrm{d}} M_{\star}}{\dot{M}_{\text {out }}}=\frac{3^{13 / 6} \sqrt{13} C_{\mathrm{R}}^{5 / 2}}{2^{8 / 3} \sqrt{\pi} Z_{0}^{6}} P_{\mathrm{R}} \approx 44 \mathrm{~d}\left(\frac{C_{\mathrm{R}}}{2.0}\right)^{4}\left(\frac{\rho_{\mathrm{d}}}{3 \mathrm{~g} \mathrm{~cm}^{-3}}\right)^{-1 / 2}$,

for bodies with mass ratio $q_{\mathrm{d}}$ at $\Delta_{\mathrm{d}}$.

\subsubsection{The pyramidal regime}

Beyond scaled distance $\Delta_{\mathrm{d}}$, many minor planets can exist simultaneously, each one migrating outwards at a rate that depends on its mass and its distance to the disc's edge. The approximate minorplanet mass-distance relation in this domain can be found by considering an idealised scenario in which bodies of equal mass are formed at a given distance at regular intervals in time. In this case, later-formed bodies will gradually catch up with earlier-formed ones, because the rate of outward migration decreases with distance. When two bodies come to within two mutual Hill radii of one another, they are assumed to undergo a perfect-merger collision, creating a body of double the mass and angular momentum. This process of catch-up then repeats itself, but with bodies that are twice the mass of the original building blocks, and that are formed half as frequently, at the location of the first merger. In turn, this also repeats itself, and so on (see Sect. S6 of CC12 for a more detailed explanation). The resulting hierarchical pattern of mergers (giving this stage the name 'pyramidal regime') yields the massdistance relation $^{7}$

$q \simeq \frac{3^{13 / 5}}{2^{16 / 5} \mathcal{T}^{3 / 5}} \Delta^{9 / 5}$

Another prediction for the pyramidal regime is that the number of minor planets $n$ follows the number-density-distance relation $\mathrm{d} n / \mathrm{d} \Delta \propto 1 / \Delta(\mathrm{CC} 12)$, meaning that for higher $\Delta$ the bodies are farther apart (specifically, they are equidistant in $\log (\Delta)$; see knotted lines in Fig. 5). To find the normalisation for this relation, we note that, at any given time, there are either one or two bodies between two consecutive locations where mergers happen, or an average of $3 / 2$ bodies per interval between merger locations. Because a merging event corresponds to a doubling of the planet mass, the $\Delta$-values corresponding to two consecutive merger locations are a factor $2^{5 / 9}$ apart (from Eq. (36)). Thus, the average number of pyramidal-regime bodies per unit of scaled distance is

$\frac{\mathrm{d} n}{\mathrm{~d} \Delta} \simeq \frac{3}{2\left(2^{5 / 9}-1\right) \Delta} \approx \frac{3.19}{\Delta}$,

and, on average, the bodies are a factor $(3 / 2)^{5 / 9} \approx 1.25$ apart in $\Delta$.

7 This can be derived from Eq. (S18) of CC12 (which gives the scaled distance of pyramidal-regime bodies when they are about to merge) by solving for $q$ and multiplying the result by $3 / 2$. The multiplication accounts for the fact that pyramidal-regime bodies on average have a mass that is a factor $3 / 2$ higher than the mass used in CC12's Eq. (S18), because mergers result in bodies of twice the mass at the same distance.
Considering Eqs. (36) and (37) in the limit $\Delta \rightarrow 0$ reveals that there is a scaled distance $\Delta_{0}$ inside of which the minor-planet number density is so high that bodies are spaced less than two mutual Hill radii $r_{\mathrm{H}}$ from one another. Inside of this distance, the pyramidal-regime relations cannot hold, because bodies would already have merged earlier. Therefore, $\Delta_{0}$ and the corresponding mass-ratio $q_{0}$ mark the formal start of the pyramidal regime. These can be computed by solving $\mathrm{d} n / \mathrm{d} r=\left(2 r_{\mathrm{H}}\right)^{-1}$, using Eqs. (36) and (37), as well as the approximation $r \simeq r_{\mathrm{R}}$ (i.e., $\Delta_{0} \ll 1$ ), which gives

$\Delta_{0} \simeq \frac{3^{23 / 6}}{2^{11 / 6}\left(2^{5 / 9}-1\right)^{5 / 2} \sqrt{\mathcal{T}}} \approx \frac{125}{\sqrt{\mathcal{T}}}$,
$q_{0} \simeq \frac{3^{19 / 2}}{2^{13 / 2}\left(2^{5 / 9}-1\right)^{9 / 2} \mathcal{T}^{3 / 2}} \approx \frac{1.13 \times 10^{4}}{\mathcal{T}^{3 / 2}}$.

In the region between $\Delta_{\mathrm{d}}$ and $\Delta_{0}$, where formally the mass-distance relation is undefined, we expect the relation to follow a trend similar to Eqs. (31) and (36). For this reason, the discrete and pyramidal regimes are connected with a dashed line in Fig. 5.

Migration via disc torques operates as long as a body has firstorder MMRs overlapping with the disc (i.e., resonances for which the mean motions obey a ratio $p+1: p$, where $p$ is a positive integer). The farthest-reaching first-order interior MMR is the 2:1 resonance. Hence, disc torques can at most move a planet out to the radius where the planet's 2:1 MMR coincides with the edge of the disc at the Roche limit. This radius and the corresponding scaled distance from the Roche limit are given by

$r_{2: 1}=2^{2 / 3} r_{\mathrm{R}} \approx 2.1 \mathrm{R}_{\odot}\left(\frac{C_{\mathrm{R}}}{2.0}\right)\left(\frac{M_{\star}}{0.6 \mathrm{M}_{\odot}}\right)^{1 / 3}\left(\frac{\rho_{\mathrm{d}}}{3 \mathrm{~g} \mathrm{~cm}^{-3}}\right)^{-1 / 3}$,

$\Delta_{2: 1}=2^{2 / 3}-1 \approx 0.587$.

Beyond $r_{2: 1}$, a planet would only have higher-order MMRs (e.g., $5: 2,3: 1$, and 4:1) overlapping with the disc and (for loweccentricity orbits) these are inefficient at transferring angular momentum (e.g., Dermott 1984). Hence, the pyramidal-regime relations cease to hold beyond $\Delta_{2: 1 .}{ }^{8}$

We can now calculate the global properties of the pyramidalregime minor-planet population when it is filled from $\Delta_{0}$ to $\Delta_{2: 1}$. The total number of bodies in the pyramidal regime is given by

$$
\begin{aligned}
& n=\int_{\Delta_{0}}^{\Delta_{2: 1}} \frac{\mathrm{d} n}{\mathrm{~d} \Delta} \mathrm{d} \Delta \simeq \frac{3}{2\left(2^{5 / 9}-1\right)} \ln \left[\frac{2^{11 / 6}}{3^{23 / 6}}\left(2^{2 / 3}-1\right)\left(2^{5 / 9}-1\right)^{5 / 2} \sqrt{\mathcal{T}}\right] \\
& \approx 32+\log _{10}\left[\left(\frac{C_{\mathrm{R}}}{2.0}\right)^{-33.09}\left(\frac{M_{\star}}{0.6 \mathrm{M}_{\odot}}\right)^{2.45}\right.\left(\frac{\rho_{\mathrm{d}}}{3 \mathrm{~g} \mathrm{~cm}^{-3}}\right)^{4.90} \\
&\left.\times\left(\frac{\Sigma_{\mathrm{R}}}{10^{4} \mathrm{~g} \mathrm{~cm}^{-2}}\right)^{-7.35}\right] .
\end{aligned}
$$

The majority of these will be small bodies close to the Roche limit. We note that this does not take into account the fact that a tightly packed system may not be dynamically stable on the timescale on which the bodies are renewed. Dynamical instabilities may lead to additional collisions and hence a lower total number of bodies with a wider spacing (see also Sect. 4.5.5). To investigate this issue in more detail would require a more thorough treatment of the gravitational interactions between the bodies (see, e.g., Hyodo et al. 2015; Salmon \& Canup 2017).

${ }^{8}$ Close to $\Delta_{2: 1}$, the relations will already become less accurate, because the derivations of $\mathrm{CC} 12$ use the approximation $r \simeq r_{\mathrm{R}}$ (i.e., $\Delta \ll 1$ ). 
The mass ratio of all the bodies in the pyramidal regime combined can be computed as

$\frac{M_{\text {pyra }}}{M_{\star}}=\int_{\Delta_{0}}^{\Delta_{2: 1}} q(\Delta) \frac{\mathrm{d} n}{\mathrm{~d} \Delta} \mathrm{d} \Delta \simeq \frac{3^{8 / 5} 5\left(2^{2 / 3}-1\right)^{9 / 5}}{2^{21 / 5}\left(2^{5 / 9}-1\right) \mathcal{T}^{3 / 5}} \approx \frac{1.29}{\mathcal{T}^{3 / 5}}$,

where we have used $\Delta_{0} \ll \Delta_{2: 1}$ in evaluating the integral. This is equivalent to about $1.7 q\left(\Delta_{2: 1}\right)$, using Eq. (36), demonstrating that the largest body in the pyramidal regime, farthest from the WD, generally holds more than half the total pyramidal-regime mass. In terms of absolute physical quantities, the total mass that can be accommodated in the pyramidal regime is

$$
\begin{aligned}
M_{\mathrm{pyra}} \simeq & \frac{5 \times 3^{2} 13^{3 / 5}\left(2^{2 / 3}-1\right)^{9 / 5} C_{\mathrm{R}}^{27 / 5}}{2^{27 / 5}\left(2^{5 / 9}-1\right) \pi^{1 / 5}} \frac{M_{\star}^{3 / 5} \sum_{\mathrm{R}}^{6 / 5}}{\rho_{\mathrm{d}}^{4 / 5}} \\
\approx & 1.6 \times 10^{25} \mathrm{~g}\left(\frac{C_{\mathrm{R}}}{2.0}\right)^{27 / 5}\left(\frac{M_{\star}}{0.6 \mathrm{M}_{\odot}}\right)^{3 / 5}\left(\frac{\rho_{\mathrm{d}}}{3 \mathrm{~g} \mathrm{~cm}^{-3}}\right)^{-4 / 5} \\
& \times\left(\frac{\Sigma_{\mathrm{R}}}{10^{3} \mathrm{~g} \mathrm{~cm}^{-2}}\right)^{6 / 5} .
\end{aligned}
$$

\subsubsection{A large planet at $r_{2: 1}$}

The pyramidal regime can only accommodate a limited amount of mass in minor planets, and this amount decreases with time as $\Sigma_{\mathrm{R}}$ goes down. Conversely, the total mass in newly formed planets $M_{\text {recyc }}$ increases with time as more disc material flows beyond the Roche limit. This accumulated mass in planets is dominated by the early mass flow through the Roche limit and therefore $M_{\text {recyc }}$ approaches a constant value at late times, which is of the order of $\dot{M}_{\text {out }}\left(t_{\text {start }}\right) t_{\text {visc }}\left[\Sigma_{\mathrm{R}}\left(t_{\text {start }}\right), r_{\mathrm{R}}\right] \simeq \pi r_{\mathrm{R}}^{2} \sum_{\mathrm{R}}\left(t_{\text {start }}\right)$. In most planet-forming disc systems, there will thus be a point in time when $M_{\text {recyc }}$ exceeds $M_{\text {pyra }}$. Once this happens, the excess material will naturally end up at $r_{2: 1}$.

Planets cannot migrate efficiently beyond $r_{2: 1}$ via disc torques, because they would have no more first-order MMRs overlapping with the disc. Other mechanisms may be considered that might transport mass beyond $r_{2: 1}$. For planetary satellites, the planet's tides can push moons further outwards (if they are located outside the planet's corotation radius). However, because WDs are so compact, the newly formed planets that we consider here are not massive enough to generate significant tidal bulges on the WD. Another possible mechanism to consider is resonance capture between two planets, which might push the outer body beyond $r_{2: 1}$, while the inner one is still inside $r_{2: 1}$, migrating outwards due to disc torques. Studies looking into this effect in satellite systems, however, find that the additional outward migration that can be achieved in this way is modest at most (Hyodo et al. 2015; Salmon \& Canup 2017).

We conclude that the outward migration of second-generation planets beyond $r_{2: 1}$ is likely to be very limited. Therefore, if a planet-forming disc is massive enough that the bodies forming out of it reach $r_{2: 1}$, most of the material flowing beyond the Roche limit will accumulate into a single large body stalled at $r_{2: 1}$. A rough estimate of the total mass in newly formed planets $M_{\text {recyc }}$ can now be made by considering angular-momentum conservation between the initial state and late times (ignoring the small angular-momentum losses due to PR drag). Assuming that all of the disc's initial angular momentum (carried by mass $M_{\text {disc }}$ at $r_{0}$ ) ends up at $r_{2: 1}$, the disc's mass-recycling efficiency is given by

$\frac{M_{\text {recyc }}}{M_{\text {disc }}} \simeq \frac{1}{2^{1 / 3}} \sqrt{\frac{r_{0}}{r_{\mathrm{R}}}} \approx 0.56\left(\frac{r_{0} / r_{\mathrm{R}}}{0.5}\right)^{1 / 2}$.
This shows that a significant portion (in many cases more than half) of the disc's original mass can be recycled into new planets. For instance, given a debris disc of initial mass $M_{\text {disc }} \sim 10^{28} \mathrm{~g}$ (i.e., the mass of a super-Earth), the largest planet could easily have the mass of the Earth.

Finally, a clear prediction can be made regarding the orbital period of the largest planet. Since this body is located around $r_{2: 1}$, where its 2:1 MMR coincides with the Roche limit, its orbital period will approximately be

$P_{2: 1}=2 P_{\mathrm{R}} \approx 11 \mathrm{~h}\left(\frac{C_{\mathrm{R}}}{2.0}\right)^{3 / 2}\left(\frac{\rho_{\mathrm{d}}}{3 \mathrm{~g} \mathrm{~cm}^{-3}}\right)^{-1 / 2}$.

\subsection{Summary of analytical findings}

Based on the analytical considerations presented in the preceding sections, we make the following tentative (to be tested numerically in the following section) conclusions about massive WD debris discs (with assumed properties as described in Sect. 2.1.2) located in the outer parts of the tidal disruption zone $\left(0.5 \lesssim r / r_{\mathrm{R}}<1\right)$ :

(i) Geometrically thin but vertically optically thick discs are gravitationally unstable (see Eqs. (10) and (11); Fig. 2), leading to self-gravity wakes that enhance the disc's effective viscosity (see Eq. (13)).

(ii) For surface mass densities higher than $\Sigma \sim 10^{3}$ to $10^{4} \mathrm{~g} \mathrm{~cm}^{-2}$ (corresponding to the initial state of discs more massive than Pluto; $M_{\text {disc }} \gtrsim 10^{25} \mathrm{~g}$ ), the disc's global evolution is dominated by viscous spreading rather than PR drag (see Eqs. (17) and (18); Fig. 3).

(iii) Discs more massive than Io $\left(M_{\text {disc }} \gtrsim 10^{26} \mathrm{~g}\right)$ will viscously spread enough for some of their mass to end up beyond the Roche limit (see Eq. (20); Fig. 4).

(iv) These massive discs can survive against viscous spreading and PR drag for longer than the age of the universe (see Eq. (23)).

Disc material flowing out beyond the Roche limit will coagulate to form a new generation of (minor) planets. These newly formed bodies will migrate outwards (due to angular momentum transfer with the disc via MMRs) and merge with one another to form larger bodies. Based on the model of CC12, we expect the new (minor) planets to have the following properties:

(i) Tens of per cent of the initial disc mass can be recycled into second-generation bodies (see Eq. (45)).

(ii) Most of the recycled mass ends up in a single object orbiting such that its 2:1 MMR coincides with the Roche limit, which means its orbital period will be of the order of $\sim 10 \mathrm{~h}$ (see Eq. (46)).

(iii) In addition, a population of some tens (see Eq. (42)) of smaller bodies is predicted in the region extending inwards from this largest planet down to the Roche limit, most of which are small bodies huddled in the innermost regions (see Eq. (37)).

(iv) Beyond a narrow region close to the Roche limit where the active formation of new bodies takes place, the distribution of masses of these bodies as a function of distance from the Roche limit roughly follows a power law with an exponent close to 2 (see Eqs. (31) and (36); Fig. 5).

(v) The ongoing formation of new bodies close to the Roche limit happens on timescales of hours, days, and months, independent on the properties of the disc (three different timescales for three different types of objects; see Eqs. (28), (34), and (35)). 


\section{NUMERICAL SIMULATIONS}

We now proceed to investigate the evolution of WD-debris-discplanet systems using numerical techniques. Numerical simulations make it possible to obtain detailed, quantitative estimates of the state of these systems as a function of time, as opposed to the predictions for their behaviour in certain limiting cases that can be found analytically. Furthermore, the numerical simulations allow us to let go of several simplifying assumptions that were necessarily made in the previous section, thereby testing the validity of the analytical predictions. Some specific improvements that were made with respect to the analytical treatment are (1) using a more complete prescription for the disc's effective viscosity, (2) computing disc-planet torques associated with individual MMRs, rather than using an average torque due to many MMRs, (3) including the back-reaction of the planets on the disc, and (4) following the evolution of individual minor planets, as opposed to starting with equal-mass building blocks. We briefly describe our numerical model and how we use it in Sect. 3.1. The results of the simulations and a comparison with the analytical predictions are presented in Sect. 3.2.

\subsection{Methods}

\subsubsection{Model description}

We employ a modified version of HYDRORINGS, a hybrid viscousdisc/discrete-body model originally developed to study the Saturnian ring-satellite system (Charnoz et al. 2010, 2011; Salmon et al. 2010). It is a one-dimensional model, which is necessary to achieve the computational speeds needed to follow the evolution of discplanet systems over multi-Gyr timescales. The code consists of two main components: (1) a viscous-evolution solver that describes the evolution of the particle disc inside the Roche limit as a continuous medium and (2) an orbital-elements tracker that models the migration and growth of discrete bodies outside the Roche limit. These two modules are coupled by prescriptions for (a) how new bodies are spawned when disc material overflows the Roche limit and (b) the subsequent interaction of these bodies with the disc through angular momentum exchange at MMRs. The model can successfully reproduce the masses and locations of many of Saturn's regular satellites and has also been tested on the formation of Earth's Moon (Charnoz et al. 2010).

The model's disc module solves the viscous diffusion equation with added external torques (e.g., Eq. (3) of Takeuchi et al. 1996) on a discretised radial grid using a second-order time-stepping method. The prescription used for the disc's effective kinetic viscosity is detailed in Sect. 2.2 of Salmon et al. (2010). This includes the translational and collisional components of viscosity (Goldreich \& Tremaine 1978; Shukhman 1984), as well as the contribution from self-gravity wakes for $Q_{\mathrm{T}}<2$ (Daisaka et al. 2001). For gravitationally unstable discs in the outer part of the tidal disruption zone, the total effective kinetic viscosity equals the one given by Eq. (13). Torques on the disc from first-order MMRs with external bodies are computed using the formalism of Meyer-Vernet \& Sicardy (1987). For this study, PR drag was added as an additional torque on the disc, computed by integrating Eq. (A5) over each radial bin. The implementation of PR drag in the code was tested by reproducing the results of Bochkarev \& Rafikov (2011, specifically, their Fig. 7). Any inwards-flowing disc material that passes through the sublimation radius $r_{\text {subl }}$ is removed from the simulation and its angular momentum is lost. This material is assumed to be converted
Table 2. Parameter values used in the numerical simulations.

\begin{tabular}{lcc}
\hline Parameter & Symbol & Value(s) \\
\hline WD effective temperature & $T_{\star}$ & $10,000 \mathrm{~K}$ \\
WD mass & $M_{\star}$ & $0.6 \mathrm{M}_{\odot}$ \\
WD radius & $R_{\star}$ & $0.0125 \mathrm{R}_{\odot}$ \\
sublimation radius & $r_{\text {subl }}$ & $0.15 \mathrm{R}_{\odot}$ \\
Roche limit & $r_{\mathrm{R}}$ & $1.3 \mathrm{R}_{\odot}$ \\
grain radius & $s$ & $1 \mathrm{~cm}^{-3}$ \\
material density & $\rho_{\mathrm{d}}$ & $3 \mathrm{~g} \mathrm{~cm}^{-3}$ \\
initial scaled disc width & $\delta$ & 0.1 \\
initial disc radius & $r_{0}$ & 0.2 to $1.2 \mathrm{R}_{\odot}$ \\
initial disc mass & $M_{\text {disc }}$ & $10^{23.5}$ to $10^{28.5} \mathrm{~g}$
\end{tabular}

to gas, which is rapidly accreted onto the WD via a gaseous viscous accretion disc (not modelled here, but see Metzger et al. 2012).

At each time step, any disc material that has spread beyond the Roche limit $r_{\mathrm{R}}$ is converted into a new minor planet, which is subsequently tracked as a discrete body (fully described by its mass, semi-major axis, and eccentricity). The semi-major axes of the bodies are modelled to evolve under the action of disc torques associated with first-order MMRs (already computed for the backreaction of the bodies on the disc; see above). Changes in the eccentricities of the bodies due to mutual perturbations are modelled using an instantaneous-close-encounter formalism (Charnoz et al. 2011). When two bodies come to within 2.2 mutual Hill radii from one another (Karjalainen 2007), they are assumed to merge into a new body whose mass is the combined mass of the two progenitors and whose orbital elements are dictated by the conservation of angular momentum. The code does not take into account resonant interactions between orbiting bodies (see discussion in Sect. S3.3 of Charnoz et al. 2010) and the treatment of tidal interactions between the planets and the central object was switched off for the present study (see Sect. 2.4.6).

\subsubsection{Setup of the model runs}

One of the main goals of this work is to investigate which WD debris discs are able to form second-generation (minor) planets and what the properties of the newly formed bodies may be. To answer these questions using our numerical model, we ran a set of simulations with a different initial disc mass $M_{\text {disc }}$ and radius $r_{0}$ for each run, keeping all other parameters fixed. Table 2 lists the parameter values we adopted. Most of our choices are motivated in Sect. 2.1, but some additional details are provided below.

For the stellar parameters, we chose values that reflect a typical WD (e.g., Kleinman et al. 2013). By adopting a fixed effective temperature for the $\mathrm{WD}$, we ignore its cooling, which will decrease the critical surface density over time. WD effective temperature roughly scales as $T_{\star} \propto t_{\text {cool }}^{-7 / 20}$ (Althaus et al. 2010), where $t_{\text {cool }}$ is the WD's cooling age. Given that a $10,000 \mathrm{~K}$ WD has a cooling age of $\sim 0.6 \mathrm{Gyr}$, the timescale on which $\Sigma_{\text {crit }} \propto T_{\star}^{4 / 3} \propto t_{\text {cool }}^{-7 / 15}$ will halve is about $\sim 2.6 \mathrm{Gyr}$.

While particle size needs to be specified in the model, and the particle size for WD debris discs is highly uncertain, the value of this parameter does not play a major role in the global evolution of the massive discs we consider (see Sect. 2.3.3). For the inwardsextending tail of material caused by PR drag (see Bochkarev \& Rafikov 2011), grain size determines the surface density but not the inward mass flow, which is the parameter that controls the disc's global evolution.

The radial grid used in the model contains 230 bins between $r_{\text {subl }}$ and $r_{\mathrm{R}}$ of width $0.005 \mathrm{R}_{\odot} \approx 3480 \mathrm{~km}$. This resolution also sets 

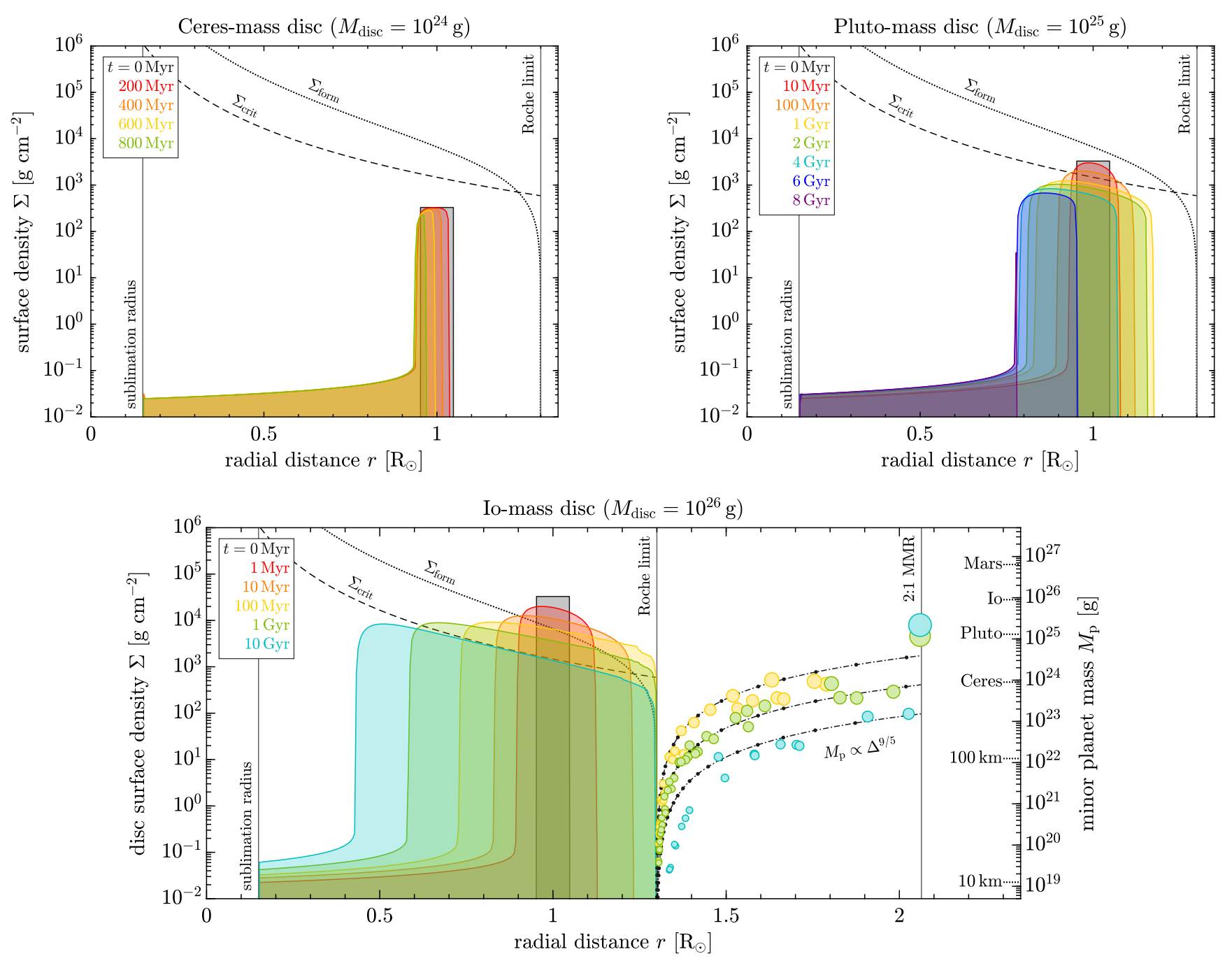

Figure 6. Three examples of the simulated evolution of a WD debris disc, using different initial masses, leading to evolution governed by different processes. The coloured lines show the surface-density profile of the discs at different points in time. The dashed black curve indicates the critical surface density $\Sigma_{\text {crit }}$ (Eq. (17)), below which disc evolution is dominated by PR drag. The dotted black curve shows the minimum initial surface density for planet formation $\Sigma_{\text {form }}$ (i.e., the equivalent of $M_{\text {form }}$, converted to a surface density using Eq. (3) with $\delta=0.1$ ). In the bottom panel, the coloured circles indicate the semi-major axes and masses of the (minor) planets that spawned from the disc, with larger symbols for more massive objects. The dash-dotted black curves show the mass-distance relation Eq. (36), scaled to roughly match the minor-planet population at different times (using, from top to bottom, $\mathcal{T}=8 \times 10^{13}, 1.2 \times 10^{15}$, and $\left.1.8 \times 10^{16}\right)$. Thick black dots in these curves indicate the typical spacing between individual objects predicted by Eq. (37). Vertical grey lines mark the sublimation radius $r_{\text {subl }}$, the Roche limit $r_{\mathrm{R}}$, and the radial distance $r_{2: 1}$ at which point a planet's 2:1 MMR coincides with the Roche limit.

the distance beyond the Roche limit at which new bodies appear. Comparing the resolution with the distances marking the domains of the various regimes of planet formation (Fig. 5) shows that only the pyramidal regime will be simulated in the vast majority of runs.

As initial radial surface-density distribution, we used a top-hat function centred at $r_{0}$ with a width given by $\delta$ (see Eq. (3)). We ran a small grid of models to investigate the effects of disc mass and radius. The initial central radius of the disc $r_{0}$ was varied between $0.2 \mathrm{R}_{\odot}$ and $1.2 \mathrm{R}_{\odot}$ in steps of $0.1 \mathrm{R}_{\odot}$. The initial disc mass $M_{\text {disc }}$ was varied between about $3 \times 10^{23} \mathrm{~g}$ and $3 \times 10^{28} \mathrm{~g}$ in logarithmic steps of a factor $\sqrt{10}$.

Each simulation is continued long enough for the particle disc to fully disappear - i.e., until all material is either locked in (minor) planets or lost at the sublimation radius. While in many cases this means simulating times that are beyond the current age of the universe, we generally only present results from the first $10 \mathrm{Gyr}$ of the simulations. Results beyond $t=10 \mathrm{Gyr}$ are used only to as- sess the validity of the analytical disc-lifetime predictions made in Sect. 2.3.5.

\subsection{Results}

\subsubsection{Three illustrative examples}

Before discussing the properties of the set of simulations as a whole, we examine the detailed results of three individual runs (shown in Fig. 6) that serve as illustrative examples for the three main (qualitatively different) types of disc evolution seen in the simulations. These runs use the same initial disc radius $\left(r_{0}=1 \mathrm{R}_{\odot}\right)$, but have different disc masses:

Low-mass disc: The top-left panel shows a disc of initial mass $M_{\text {disc }}=10^{24} \mathrm{~g} \sim 1$ Ceres mass, whose initial surface mass density is below $\Sigma_{\text {crit }}$. The evolution of this disc is governed almost entirely 
by PR drag and can be described well by the results of Bochkarev \& Rafikov (2011, see Sect. 2.3.2).

Intermediate-mass disc: The top-right panel is for an initial mass $M_{\text {disc }}=10^{25} \mathrm{~g} \sim 1$ Pluto mass. The disc's initial surface density is above $\Sigma_{\text {crit }}$, but its initial mass is still below $M_{\text {form }}$. The disc initially spreads viscously (until about $t=1 \mathrm{Gyr}$ ), but before the outer edge has reached the Roche limit the surface density drops below the critical level and PR drag takes over the further evolution.

High-mass disc: The bottom panel is for a disc of initial mass $M_{\text {disc }}=10^{26} \mathrm{~g} \sim 1$ Io mass, which is above $M_{\text {form }}$. Its evolution is dominated by viscous spreading for most of the simulation. At about $t=30 \mathrm{Myr}$, the disc spreads beyond the Roche limit and new minor planets start being formed. These migrate outwards and merge with one another, roughly following the pyramidal-regime mass-distance relationship (Eq. (36)). Eventually most of the minor planets coagulate into a Pluto-mass body orbiting at $r_{2: 1}$. Around $t=10 \mathrm{Gyr}$, the disc decouples from the Roche limit and the formation of new bodies stops. For some time after this point, however, the existing small bodies close to the Roche limit still migrate outwards through interaction with the retreating disc. This results in a deviation from the analytical mass-distance relation towards the end of the run (see also Sect. 3.2.3).

\subsubsection{Main results from the full set of simulations}

A broad overview of the simulation results is given by Fig. 7, in which several key quantities extracted from the simulations are mapped against the initial disc masses and radii of the runs. Based on these and other results from the simulations, we make the following observations and inferences:

(i) The analytical approximation for the minimum disc mass for planet formation $M_{\text {form }}$ (Eq. (20)) closely matches the line separating systems with and without planets in the numerical results. The small discrepancy at $r_{0}>1 \mathrm{R}_{\odot}$ can be explained by the non-zero initial width of the simulated discs: if the outer parts of a simulated disc start off close enough to the Roche limit, the disc can form (minor) planets despite having an initial mass below $M_{\text {form }}$.

(ii) A large fraction of the initial disc mass can be converted into planets. For the highest initial disc masses we consider $\left(M_{\text {disc }} \gtrsim\right.$ $10^{28} \mathrm{~g}$ ), this recycled mass fraction $M_{\text {recyc }} / M_{\text {disc }}$ roughly converges to the values predicted by Eq. (45). A small discrepancy between the analytical and numerical results is caused by the fact that some angular momentum is lost in the simulations by material flowing inside the sublimation radius.

(iii) In almost all of the runs, most of the planet mass eventually accumulates in a single large body orbiting at $r_{2: 1}$. Only a few systems (all with initial disc masses close to $M_{\text {form }}$ ) have a largest body containing less than $90 \%$ of the total planet mass at the end of the run. In only two of these exceptions (the two empty cells in the top-right panel of Fig. 7), the largest body does not reach $r_{2: 1}$. These two systems populate only part of the pyramidal regime before PR drag terminates their planet formation.

(iv) The time at which the first bit of disc material reaches the Roche limit and planets start forming $t_{\text {start }}$ varies dramatically, from a fraction of a year (for the highest mass disc starting closest to the Roche limit), to several Gyr (for initially compact discs with masses close to $M_{\text {form }}$ ). Its scaling with initial disc mass and radius roughly resembles that of the viscous timescale (Fig. 3). The low values demonstrate that small bodies located just outside the Roche limit can form very quickly.

(v) The time at which the total mass in planets $M_{\text {recyc }}$ has reached half its final value (referred to as the planet-formation timescale in Fig. 7) is relatively short in most runs (compared with, e.g., the total length of time that planet formation can remain operating, which is often longer than the age of the universe; specifically, for massive discs it is roughly $t_{\text {crit }}\left(r_{\mathrm{R}}\right) \approx 39 \mathrm{Gyr}$; see Sect. 2.3.5). This shows that the final planet mass $M_{\text {recyc }}$ is dominated by the early stages of planet formation. The reason for this is that the planet-forming mass flow through $r_{\mathrm{R}}$ is highest at time $t_{\text {start }}$ and subsequently declines (because $\Sigma_{\mathrm{R}}$ goes down; see Sects. 2.4.1 and 2.4.6).

(vi) Disc lifetimes follow the predictions made in Sect. 2.3.5, converging to Eq. (23) for initial disc masses of $M_{\text {disc }} \gtrsim 10^{28} \mathrm{~g}$, and following Eq. (21) in simulations far enough below the dashed $M_{\text {crit }}$ line. Most planet-forming discs can survive viscous and PRdrag evolution for longer than the age of the universe.

(vii) For low-mass systems, the inward mass flow rate at the sublimation radius (which we assume to be approximately equal to the mass accretion rate onto the WD) are consistent with the PRdrag-induced mass flow given by Eq. (15) and predicted in more detail by Bochkarev \& Rafikov (2011). In simulations with initial disc masses above $M_{\text {crit }}$ (dashed line), viscous spreading moves the disc's inner edge inwards, which increases the mass flow due to PR drag slightly. A strong enhancement of the mass accretion rate due to viscous spreading (reaching $\gtrsim 10^{9} \mathrm{~g} \mathrm{~s}^{-1}$ ) is only seen in discs with initial masses of $M_{\text {disc }} \gtrsim 10^{27} \mathrm{~g}$. In these cases, the mass flow at $r_{\text {subl }}$ is caused directly by viscous spreading. This confirms the conclusion of Metzger et al. (2012, their Appendix A) that, as an explanation for the highest accretion rates of polluted WDs, viscous spreading requires disc masses comparable to that of a terrestrial planet.

\subsubsection{Minor planet properties}

Finally, we review the detailed properties of the simulated minorplanet populations and compare them to the predictions of the analytical model presented in Sect. 2.4. Figure 8 shows this comparison for a representative subset of the runs. This shows that the numerical results generally match the analytical predictions for dimensionless disc evolution timescales of $\mathcal{T} \sim 10^{9}$ to $10^{15}$, as expected. In several instances, however, deviations occur where the numerical results follow a steeper slope than the analytical predictions and/or extend below the $\mathcal{T}=10^{15}$ line. Further inspection of the simulation results reveals that this happens when the outward mass flow is quenched, which can occur for two reasons. (1) When a strong MMR of a massive body is located in the outermost parts of the disc, the back-reaction of this body on the disc can push the outer edge away from the Roche limit (e.g., the orange circles for $t=10^{4} \mathrm{yr}$ in the top-right panel). (2) At late times, PR drag starts to reverse the mass flow at the Roche limit (e.g., the purple and pink circles for $t=10^{9}$ and $10^{10} \mathrm{yr}$ in the bottom-right panel).

\section{DISCUSSION}

\subsection{The occurrence of massive WD debris discs}

In the preceding sections, we found that viscously spreading WD debris discs will produce new (minor) planets when they spread beyond the Roche limit, but that this only happens for very massive discs (roughly $M_{\text {disc }} \gtrsim 10^{26} \mathrm{~g}$; see Fig. 4). This brings up the question whether discs that are sufficiently massive (to reach the Roche limit) actually exist. As mentioned in Sect. 1, WD debris discs are 

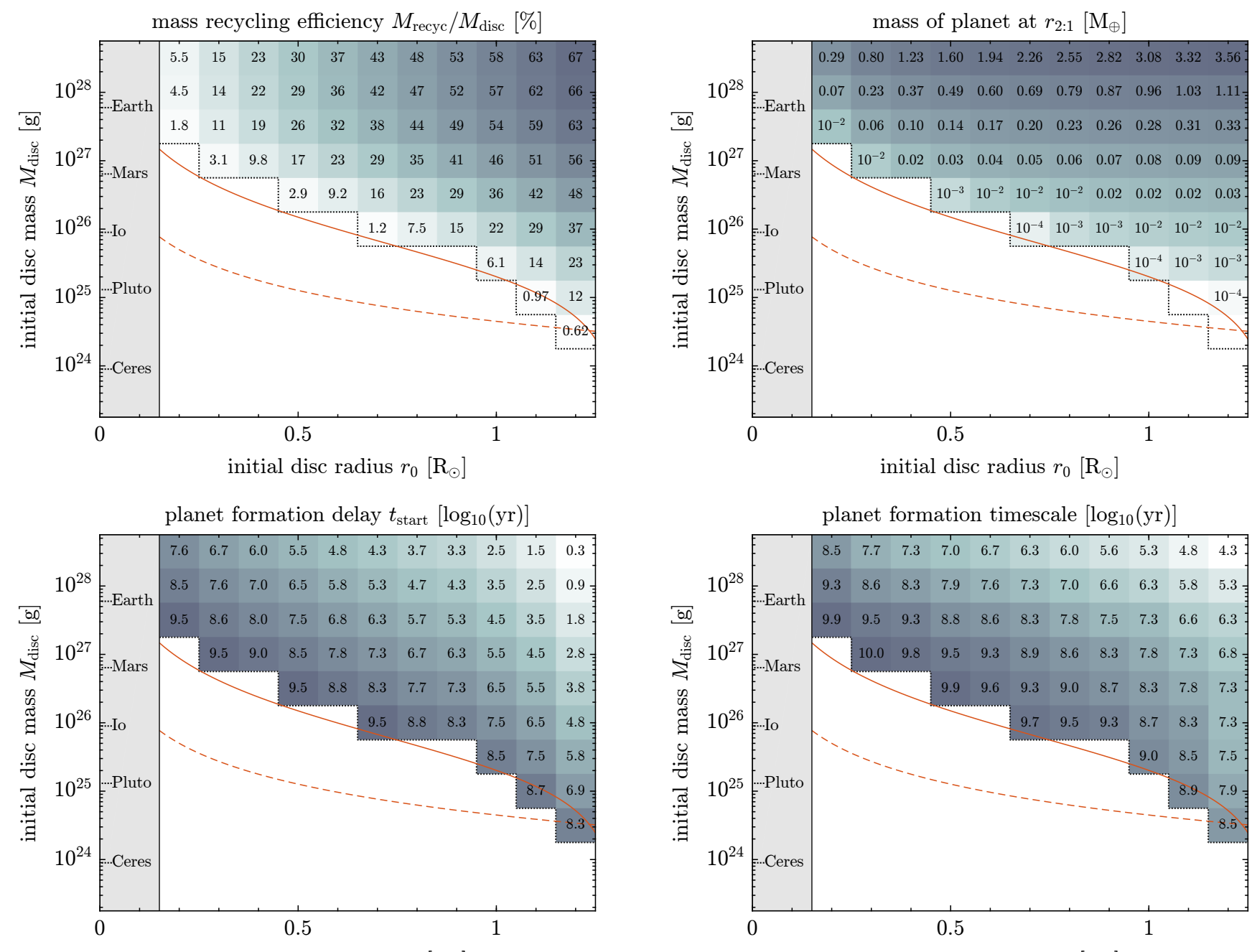

planet formation timescale $\left[\log _{10}(\mathrm{yr})\right]$

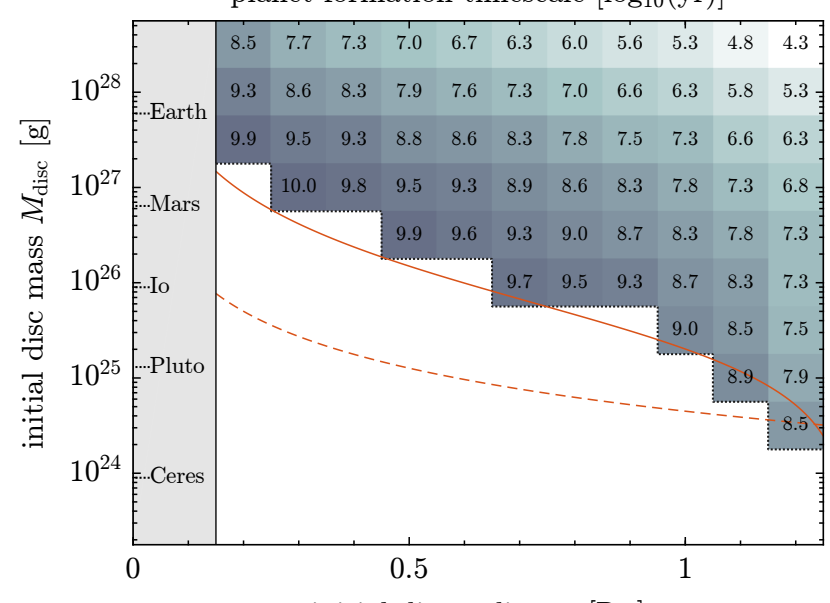

initial disc radius $r_{0}\left[\mathrm{R}_{\odot}\right.$
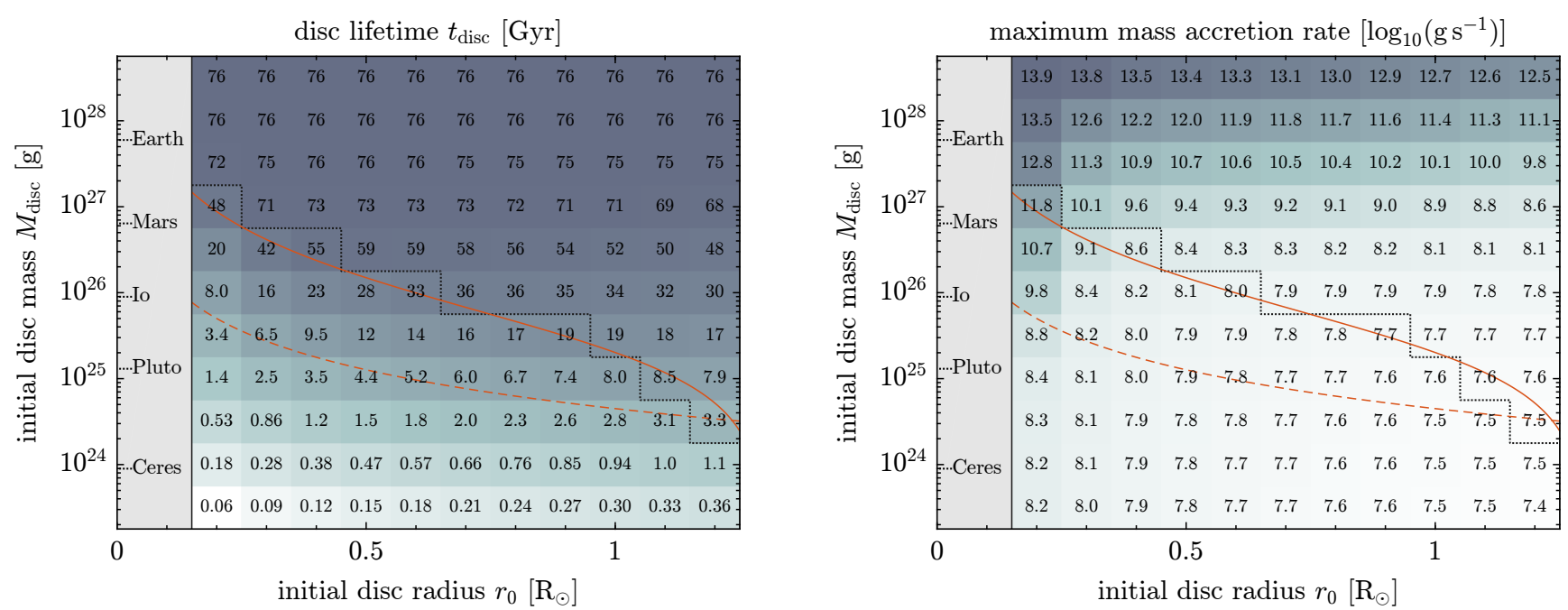

Figure 7. Key results of the numerical simulations, shown as a function of the initial central radius of the disc $r_{0}$ and its initial mass $M_{\mathrm{disc}}$. Each individual run is represented by a coloured cell with a number inside. The colouring is included to guide the eye; it contains the same information as the numbers. A dotted black line separates runs in which (minor) planets are formed from those in which this does not happen. The red lines are the same as those in Fig. 4: they show the analytical approximations of the minimum disc mass for planet formation $M_{\text {form }}$ (solid line) and the critical disc mass $M_{\text {crit }}$ above which viscous spreading initially dominates the disc's evolution (dashed line). Top-left panel: Total mass in (minor) planets after $10 \mathrm{Gyr}$, scaled to the initial disc mass. Top-right panel: Mass of the body at $r_{2: 1}$ after $10 \mathrm{Gyr}$, in Earth masses. Middle-left panel: Time at which the first planet is formed. Middle-right panel: Time at which half the final planet mass is formed. Bottom-left panel: Time until all of the disc's initial mass is converted into new (minor) planets and/or accreted onto the WD. Bottom-right panel: Highest absolute mass flow at the sublimation radius, averaged over 1 Myr, recorded in the first $10 \mathrm{Gyr}$ of the simulation. 

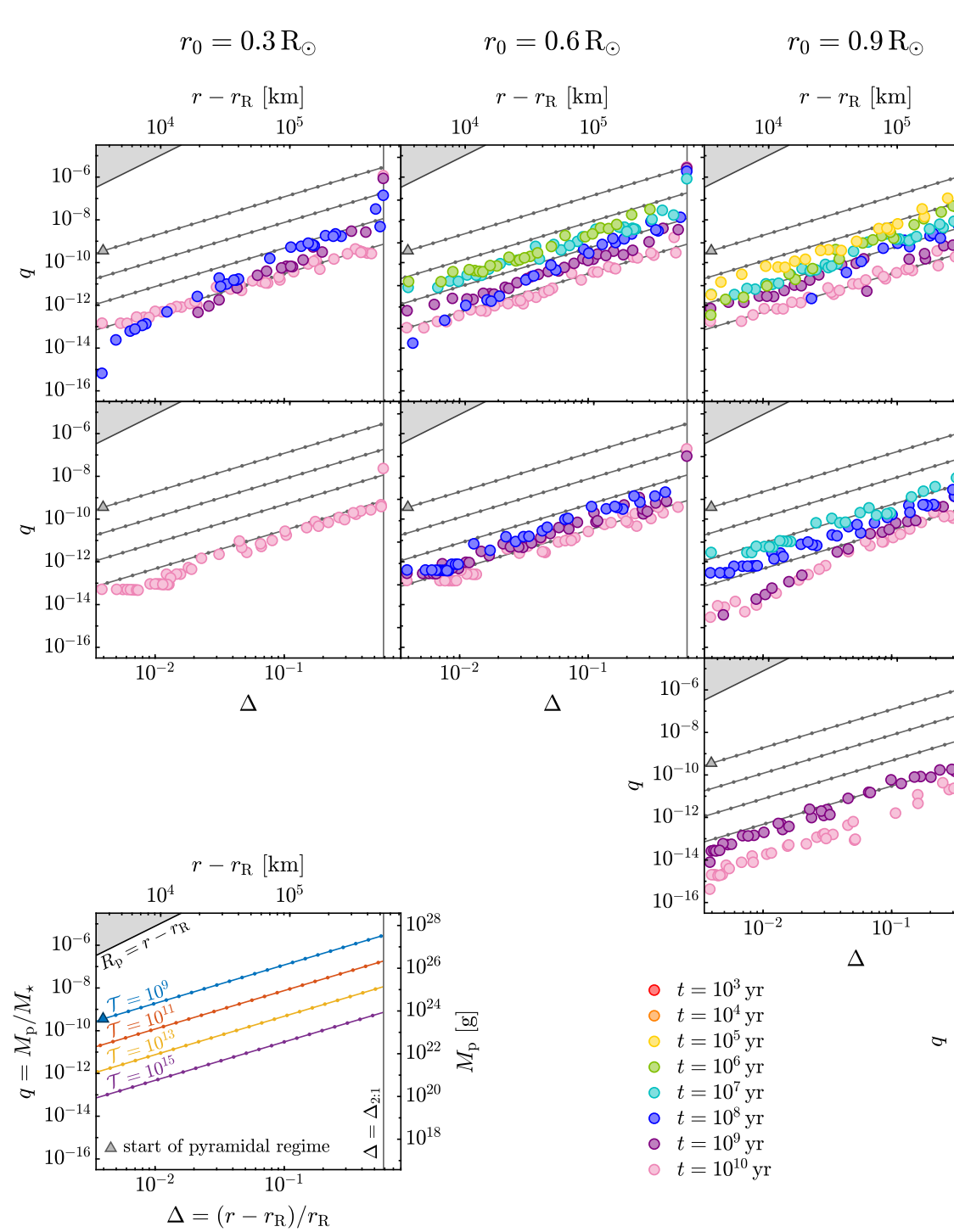

$$
\begin{aligned}
& r_{0}=1.2 \mathrm{R}_{\odot} \\
& \begin{array}{lrrr}
10^{4} & 10^{5} & 10^{4} & r-r_{\mathrm{R}}[\mathrm{km}] \\
\hline
\end{array}
\end{aligned}
$$
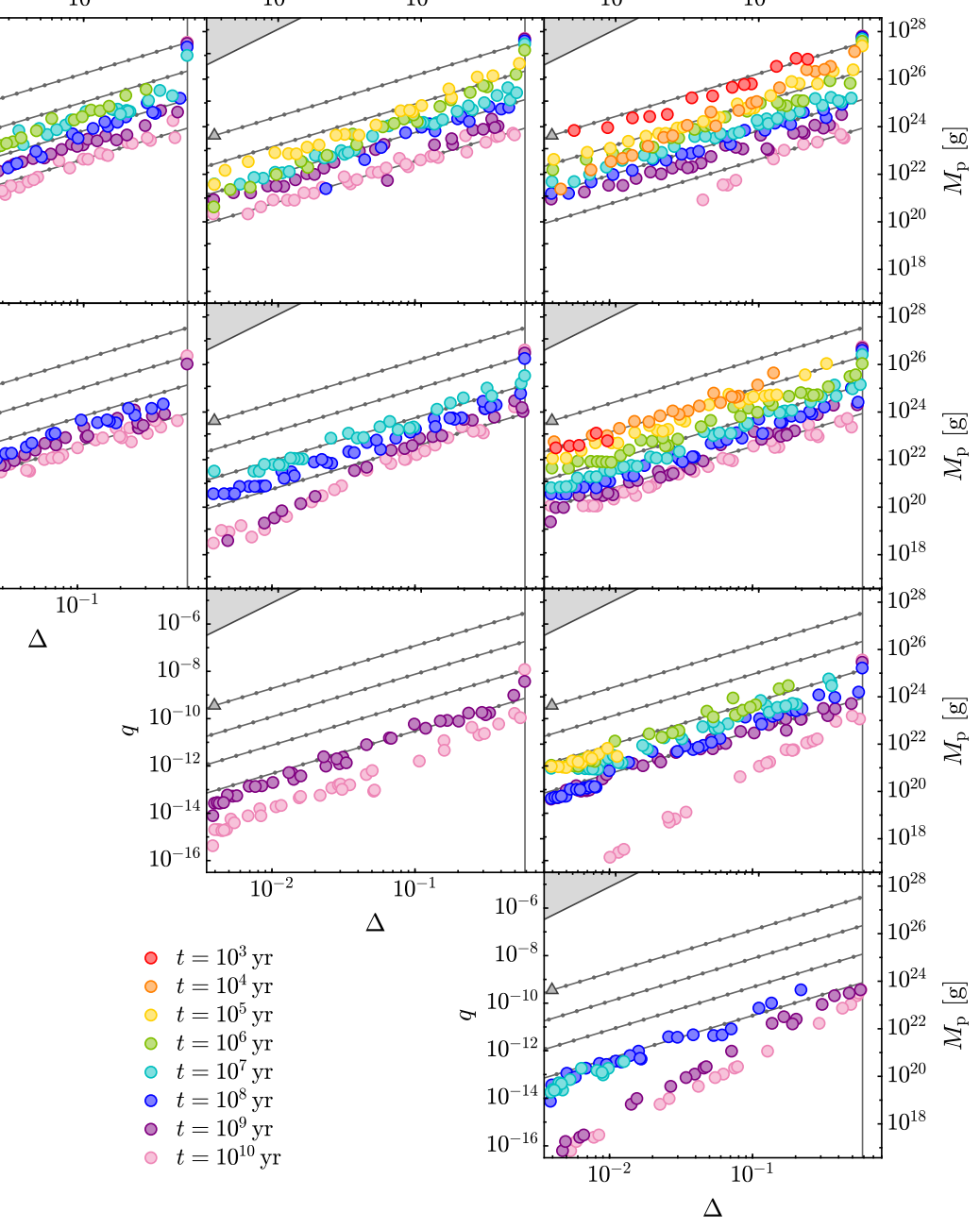

Figure 8. Properties of the (minor) planets seen in a subset of the numerical simulations, overplotted on the analytical predictions found in Sect. 2.4. Each panel shows results from a single simulation, whose initial disc radius $r_{0}$ and disc mass $M_{\text {disc }}$ can be found along the top and right-hand side of the figure, respectively. The coloured circles represent individual (minor) planets, indicating their mass $M_{\mathrm{p}}$ and distance from the Roche limit $r-r_{\mathrm{R}}$ (or, in terms of dimensionless parameters, their mass ratio $q=M_{\mathrm{p}} / M_{\star}$ and scaled distance $\left.\Delta=\left(r-r_{\mathrm{R}}\right) / r_{\mathrm{R}}\right)$. Different colours correspond to different times in the simulation, as labelled by the legend at the bottom-centre of the figure. In all panels, a vertical grey line marks the scaled distance $\Delta_{2: 1}$ at which a planet's $2: 1$ MMR coincides with the Roche limit. The grey, knotted lines in each panel are pyramidal-regime mass-distance relations for a range of plausible disc properties. They are the same for each panel, and a coloured, labelled version of these lines is reproduced in a separate panel in the bottom-left corner of the figure. This separate panel corresponds to a part of Fig. 5; see the caption of that figure for further information. The bottom-left section of the figure does not contain any panels with simulation results, because the runs with the corresponding initial parameters did not produce any minor planets.

usually assumed to have masses comparable to Solar-System asteroids $\left(M_{\text {disc }} \lesssim 10^{23} \mathrm{~g}\right.$ ), but the atmospheric-pollution masses on which this number is based only give lower limits on the disc mass. There are several reasons to believe that there could also be discs with masses in the range $10^{26}$ to $10^{28} \mathrm{~g}$ (i.e., the mass of a dwarf planet, major moon, or terrestrial planet):

(i) Some heavily polluted WDs with helium-dominated atmospheres also contain significant amounts of hydrogen (which does not sink down). In the case of one of these, GD 362, it has been shown that this pattern of pollution can be explained by the accretion of a terrestrial-planet-mass object containing some water (Jura et al. 2009b). A similar scenario may account for other WDs ex- hibiting the combination of heavy atmospheric metal and hydrogen pollution, although the hydrogen may also have been accumulated through the accretion of multiple smaller water-rich bodies over an extended period of time (see also Raddi et al. 2015; Gentile Fusillo et al. 2017).

(ii) Theoretical studies of planetary-system evolution show that in some cases planets have pericentre excursions below the Roche limit ( $\sim 0.1$ of simulated systems; Mustill et al. 2014; Veras et al. 2016a). Furthermore, in unstable planetary systems, massive moons can be liberated from their host planet (Payne et al. 2016) and scattered into the Roche limit (Payne et al. 2017), although the efficiency of this process has yet to be quantified.

(iii) For WDs with binary companions, mechanisms have been 
proposed to drive small bodies from an outer planetesimal belt down to the WD (Bonsor \& Veras 2015; Hamers \& Portegies Zwart 2016; Stephan et al. 2017). These mechanisms would apply equally to planets.

(iv) The tidal disruption of a planet-sized body around a WD might be necessary to explain the properties of the interstellar asteroid 1I/2017 'Oumuamua (Rafikov 2018). In such a tidal disruption event, only a minority $(\$ 30 \%)$ of the debris is ejected to interstellar space, while the rest remains bound to the WD.

An important question following from these points is whether the tidal disruption of a massive body would result in a debris disc with the flat, compact geometry that we have used as the starting point of our analysis. Studies considering the formation of planetary rings from the tidal disruption of a comet or Kuiper Belt object argue that this is possible (Dones 1991; Hyodo et al. 2017), but recent studies focussing on WDs have brought forward problems with the flat disc geometry (Kenyon \& Bromley 2017a,b). The problem of disc formation and geometry is more general to the understanding of circumstellar dust around WDs and extends beyond the scope of this paper. We reiterate that our study does not presuppose any particular formation scenario for the disc. All conclusions regarding second-generation planets apply equally if the required discs form via a different pathway.

\subsection{Recycled planets with or without a remnant debris disc?}

Given the long lifetime of massive WD debris discs (see Sect. 2.3.5), any second-generation exoplanets produced via the recycling mechanism are expected to be accompanied by (a remnant of) the disc that produced them. For this reason, WDs exhibiting IR excess may be the most promising targets to search for recycled planets. For the same reason, the recycling mechanism may be expected to not occur frequently, because the discs that produce planets would also give rise to prominent IR excesses, and the fraction of WDs with a detectable IR excess is fairly limited (1 to $3 \%$ for WDs with ages $t_{\text {cool }} \lesssim 0.5 \mathrm{Gyr}$; Farihi et al. 2009; see also Bonsor et al. 2017).

On the other hand, planets may occur without an accompanying remnant debris disc if, after the planets have already been formed, the disc is destroyed by some mechanism unaccounted for in our analysis. Possible examples of such mechanisms are the runaway accretion of the disc induced by gas drag (Rafikov 2011a; Metzger et al. 2012) and a vaporising collision between the disc and a newly-inwards-scattered body from the star's original planetary system (Jura 2008). In the case of disc destruction by run-away accretion, second-generation planets may be expected to have formed before the disc is gone, because the delay time for run-away accretion is of the order of 0.1 to $10 \mathrm{Myr}$ (although this estimate is highly uncertain; see Eq. (59) of Metzger et al. 2012), while the formation of planets starts earlier for many planet-producing discs (see Fig. 7, middle-left panel).

If a massive disc is destroyed after some second-generation planets have already been formed, the planets may act as a longterm (Gyr-timescale) reservoir of mass, located just outside the Roche limit and available for future accretion onto the WD. Two possible Solar-System analogues of this situation are the Uranian and Neptunian satellite systems, both of which may have formed out of ancient massive planetary rings that have since disappeared (CC12). In a scenario in which WD debris discs are generally only created very early in a WD's life (possibly as a result of some aspect of post-main-sequence evolution) and then rapidly destroyed (e.g., by run-away accretion), the long-term storage of mass in secondgeneration planets could be the explanation for the occurrence of atmospheric metal pollution in older WDs. A testable prediction for this scenario is that a large fraction of WDs (at least as high as the fraction of WDs that show pollution; i.e., tens of per cent) should be orbited by (minor) planets just outside the Roche limit.

\subsection{Planets in the habitable zone of WDs}

A WD's habitable zone (in which a terrestrial planet can harbour liquid water on its surface) is located at a few Solar radii from the star, gradually moving inwards as the WD cools (Agol 2011; Barnes \& Heller 2013). Since the region close to a WD ( $r \lesssim 1 \mathrm{AU})$, including the habitable zone, has been cleared during the star's post-main-sequence evolution, no planets are generally expected here (Mustill \& Villaver 2012), except maybe massive gas giants that have survived a common-envelope event (Nordhaus \& Spiegel 2013). If the recycling process studied in this paper occurs, however, it predicts the existence of low-mass $\left(M_{\mathrm{p}} \ll 1 \mathrm{M}_{\text {Jup }}\right)$ planets in compact $(r \ll 1 \mathrm{AU})$ orbits around WDs.

Interestingly, the orbital radius that our model predicts for the largest planet, $r_{2: 1}$ (see Eq. (40); Sect. 2.4.6), is squarely in the middle of the WD 'continuously habitable zone' identified by Agol (2011). This is defined as the range of distances that remain habitable for an extended period of time (e.g., at least $3 \mathrm{Gyr}$ ). Specifically, for a planet orbiting a $0.6 \mathrm{M}_{\odot} \mathrm{WD}$ at $r_{2: 1} \approx 2.1 \mathrm{R}_{\odot}$, the habitable zone starts to coincide with the planet's location when the WD has cooled to an effective temperature of about $T_{\text {eff }} \approx 6000 \mathrm{~K}$, corresponding to a WD age of around $2 \mathrm{Gyr}$, and it has moved too far inwards at $T_{\mathrm{eff}} \approx 4000 \mathrm{~K}$, at an age of about $10 \mathrm{Gyr}$ (Agol 2011).

This situation raises further questions regarding the potential habitability of planets formed through the recycling mechanism: Would any volatiles survive the processing of material in the disc? If not, could enough water be delivered onto the planet from outside reservoirs later on? Although a detailed consideration of these issues is beyond the scope of this paper, we now briefly discuss the question of water delivery.

Veras et al. (2014b) show that the trace amounts of hydrogen that are observed in the atmospheres of helium-dominated WDs can be explained by the accretion of water-rich comets from remnant Oort-cloud-type reservoirs. Over a timespan of $\sim 10 \mathrm{Gyr}$, this mechanism could provide $10^{22}$ to $10^{25} \mathrm{~g}$ in $\mathrm{H}$ onto the WD (see also Fig. 5 of Raddi et al. 2015), corresponding to $10^{23}$ to $10^{26} \mathrm{~g}$ in $\mathrm{H}_{2} \mathrm{O}$. Assuming the WD's catchment area corresponds to the cross-section of its tidal disruption sphere, a planet can accrete a fraction on the order of $\left(R_{\mathrm{p}} / r_{\mathrm{R}}\right)^{2}$ of this mass, ignoring gravitational focussing. For an Earth-sized body, this would yield about $10^{19}$ to $10^{22} \mathrm{~g}$ in water (equivalent to $10^{-5}$ to $10^{-2}$ times the mass of Earth's oceans), some fraction of which will stay on the planet during the impact process, depending on the size and velocity of the impactors (see, e.g., Shuvalov 2009; Schlichting \& Mukhopadhyay 2018; Kral et al. 2018). Whether this amount of water would be sufficient for the emergence of life is another question that remains to be answered.

Finally, if habitable planets around WDs exist and they can be studied in transit, they would make uniquely favourable targets for attempts to detect biomarkers (Loeb \& Maoz 2013). Our study contributes to this intriguing subject by showing that the formation of planets in the habitable zone of WDs is possible, indeed likely, for certain conditions. 
Table 3. Published results of transit surveys searching for WD planets

\begin{tabular}{ccccc}
\hline Ref. & Using & WDs & $\begin{array}{c}\text { Cadence } \\
{[\mathrm{min}]}\end{array}$ & $\begin{array}{c}\text { Min. detectable } R_{\mathrm{p}} \\
{\left[\mathrm{R}_{\oplus}\right]}\end{array}$ \\
\hline F11 & SuperWASP & 194 & 8 to 10 & 0.4 to 1 \\
F14 & Pan-STARRS & 1718 & 4 & 1 to 2 \\
S16 & HST/COS & 7 & 0.25 & 0.08 to 0.7 \\
B16 & DECam & 111 & 1.5 & 0.3 to 1 \\
V18 & K2 & 1148 & 1 or 30 & 0.125 to 1 \\
W18 & ARCSAT & 5 & 1 & 0.3 to 0.5 \\
\hline
\end{tabular}

References. B16: Belardi et al. (2016); F11: Faedi et al. (2011); F14: Fulton et al. (2014); S16: Sandhaus et al. (2016); V18: van Sluijs \& Van Eylen (2018); W18: Wallach et al. (2018).

\subsection{Observational constraints and prospects}

The existence of planets in compact orbits around WDs is an interesting conjecture that warrants to be tested observationally. Given the small orbital radii and large planet-to-star size ratio of the predicted planets, transit surveys are likely to be a fruitful strategy for constraining how frequently they occur. The planets can cause very deep transits or even complete occultations of their host star, recurring at periods less than a day. Thus, even relatively low-precision surveys (like some present-day ground-based efforts) are capable of detecting them (see Faedi et al. 2011; Agol 2011). Because the durations of WD planetary transits are relatively short (typically 1 to 3 min for the planets we consider; e.g., Faedi et al. 2011), the sensitivity of such surveys to small planets will depend critically on the exposure time and/or observational cadence used.

Several transit surveys have been conducted so far to search for planets around WDs (see Table 3 for some of their specifics), but no solid-body planetary companions have yet been found (irregular transits were discovered for WD 1145+017, probably caused by clouds of dust; see Sect. 4.5). Overall, the constraints on the occurrence rate of small planets around WDs are still relatively weak. While the presence of Mars-to-Earth-sized bodies in 10-h orbits around half of all WDs or more can be ruled out (van Sluijs \& Van Eylen 2018), a high-cadence or high-precision transit survey with a larger sample size would be needed to put stronger limits on the occurrence rate of the smaller $\left(R_{\mathrm{p}}<0.5 \mathrm{R}_{\oplus}\right)$ bodies predicted in this paper. Given that the geometric transit probability of these planets is of the order of $1 \%$ (e.g., Faedi et al. 2011), a detection would likely require a sample size of at least a few hundreds divided by the occurrence rate (see also Agol 2011).

To evaluate the prospects of future transit surveys to put meaningful constraints on the occurrence rate of (or to detect) planets created via the recycling mechanism, we now make estimates of the transit properties of these bodies. For a planet of radius $R_{\mathrm{p}}$ on a circular orbit with radius $r$, the geometric transit probability is

$p_{\mathrm{tr}}=\frac{R_{\mathrm{p}}+R_{\star}}{r}$.

If, additionally, the planet's orbit is oriented edge-on (i.e., zero impact parameter), the transit depth $\delta_{\text {tr }}$ and full duration $D_{\text {tr }}$ are given by (e.g., Faedi et al. 2011)

$\delta_{\text {tr }}= \begin{cases}\left(\frac{R_{\mathrm{p}}}{R_{\star}}\right)^{2} \approx 0.54\left(\frac{R_{\mathrm{p}}}{1 \mathrm{R}_{\oplus}}\right)^{2}\left(\frac{R_{\star}}{0.0125 \mathrm{R}_{\odot}}\right)^{-2} & \text { for } R_{\mathrm{p}}<R_{\star} \\ 1 & \text { for } R_{\mathrm{p}} \geq R_{\star}\end{cases}$

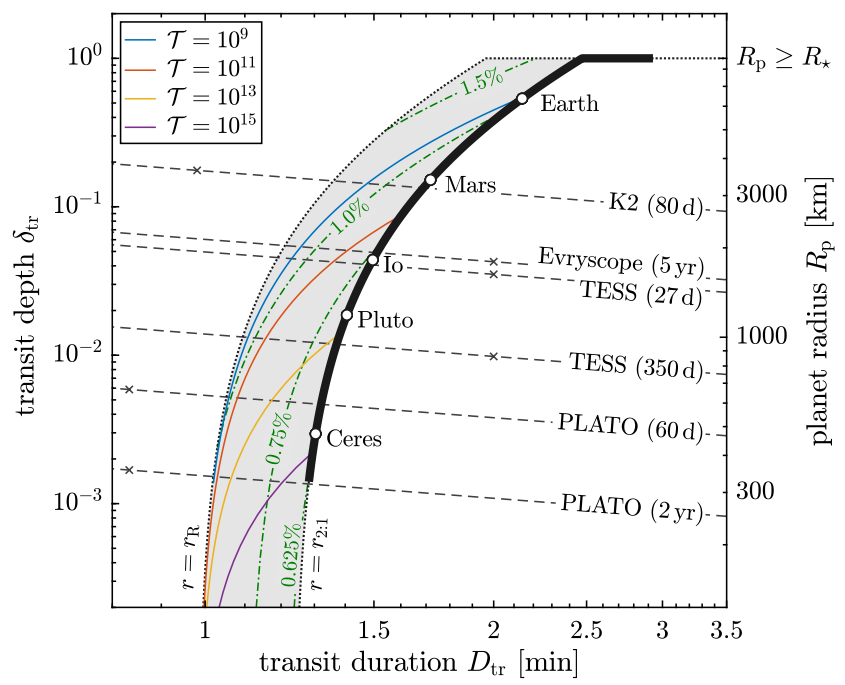

Figure 9. Transit properties of recycled planets and their detectability. The shaded area marks the part of transit-depth-and-duration space in which transits by recycled planets can in principle occur. It is bounded by the dotted black curves, which correspond to bodies orbiting at radii $r_{\mathrm{R}}$ and $r_{2: 1}$, and extends out to longer transit durations along the horizontal dotted line at a transit depth of unity (for planets that are larger than the star). The thick black segment indicates the transit properties of the $r_{2: 1}$ bodies seen in the numerical simulations (see Fig. 7, top-right panel). The four coloured solid curves correspond to pyramidal-regime bodies for different disc properties, parametrised by the disc's dimensionless Roche-limit viscous evolution timescale $\mathcal{T}$ (see Eq. (30) and Fig. 5). Lines of constant geometric transit probability are shown by green dash-dotted curves (Eq. (47)). Transit parameters were computed assuming a WD with mass $M_{\star}=0.6 \mathrm{M}_{\odot}$ and radius $R_{\star}=0.0125 \mathrm{R}_{\odot}$, and planets on edge-on orbits (i.e., zero impact parameter). To convert planet masses to radii, we assume a mean density of $3 \mathrm{~g} \mathrm{~cm}^{-3}$ for all bodies. Several Solar-System bodies are indicated on the black curve, showing their transit properties if they orbited the WD at $r_{2: 1}$. The dashed grey lines show detection limits of some ongoing and future transit surveys to achieve a $\mathrm{S} / \mathrm{N}$ of 10 for planets orbiting at $r_{2: 1}$ (Eq. (50); using the properties listed in Table 4). For objects in more compact orbits, these lines overestimate the detection limits only slightly (see footnote 9). An $\times$-symbol marks the (possible) cadence of each survey. For transits with durations below the cadence, the detection limits will be somewhat higher because the transit signal will be diluted.

$$
\begin{aligned}
D_{\mathrm{tr}} & =2 \sqrt{\frac{r}{G M_{\star}}}\left(R_{\mathrm{p}}+R_{\star}\right) \\
& \approx 2.1 \min \left(\frac{M_{\star}}{0.6 \mathrm{M}_{\odot}}\right)^{-1 / 2}\left(\frac{r}{2 \mathrm{R}_{\odot}}\right)^{1 / 2}\left(\frac{R_{\mathrm{p}}+R_{\star}}{1 \mathrm{R}_{\oplus}+0.0125 \mathrm{R}_{\odot}}\right) .
\end{aligned}
$$

The detectability of a planet using the transit method depends on the signal-to-noise ratio $(\mathrm{S} / \mathrm{N})$ of the transit signal, which can be estimated as (e.g., Rowe et al. 2014)

$\mathrm{S} / \mathrm{N}=\sqrt{N_{\text {in }}} \frac{\delta_{\text {tr }}}{\sigma_{\text {phot }}}$.

Here, $N_{\text {in }}$ is the total number of in-transit measurements (i.e., summed over all observed transit events) and $\sigma_{\text {phot }}$ is the photometric precision on a single measurement. The minimum $\mathrm{S} / \mathrm{N}$ needed for reliable transit detection depends on the desired detection efficiency and false-alarm probability. Following Rowe et al. (2014), we adopt $\mathrm{S} / \mathrm{N} \geq 10$.

Figure 9 shows the transit properties of the bodies predicted by the recycling mechanism, together with rough estimates of the 
Table 4. Properties of some current and future transit surveys targeting WDs

\begin{tabular}{|c|c|c|c|c|c|c|}
\hline Survey & $\begin{array}{l}\text { Cad. } \\
\text { [min] }\end{array}$ & Mag. & $\begin{array}{l}\text { Prec. } \\
{[\%]}\end{array}$ & $\begin{array}{c}\text { Basel. } \\
\text { [d] }\end{array}$ & $\begin{array}{c}\text { Sky cov. } \\
{[\%]}\end{array}$ & WDs \\
\hline K2 & 1,30 & 19 & 3 & 80 & 5 & 2000 \\
\hline Evryscope & 2 & 16 & 2 & (note) & 100 & $1000 \mathrm{~s}$ \\
\hline TESS & 2,30 & 15 & 0.5 & $\begin{array}{c}27 \\
350\end{array}$ & $\begin{array}{c}85 \\
2\end{array}$ & $\begin{array}{c}1000 \\
10 \mathrm{~s}\end{array}$ \\
\hline PLATO & 1,10 & 16 & 0.08 & $\begin{array}{c}60 \\
730\end{array}$ & $\begin{array}{c}25 \\
5\end{array}$ & $\begin{array}{c}100 \mathrm{~s} ? \\
10 \mathrm{~s} ?\end{array}$ \\
\hline
\end{tabular}

Columns. Cad.: Possible observational cadences. Mag.: Typical magnitude of WDs that can be observed (in the instrument's native waveband). Prec.: Expected photometric precision in $1 \mathrm{~h}$ of observations at the listed magnitude. Basel.: Temporal baseline over which targets are monitored. Sky cov.: Fraction of the sky that is monitored for the listed baseline or longer. WDs: Estimated number of WDs of the listed magnitude or brighter that will be monitored (still very uncertain for PLATO).

References. K2: Howell et al. (2014); http: //www. k2wd.org (J. J. Hermes 2017). Evryscope: Law et al. (2015). TESS: Ricker et al. (2015); Raddi et al. (2017); Stassun et al. (2017); TASC target lists of WG-8 Compact pulsators (https://tasoc.dk/info/targetlist.php). PLATO: Rauer et al. (2014), PLATO Definition Study Report (Red Book).

Note. Being a ground-based survey, Evryscope does not have a pre-defined temporal baseline in the 'constant-staring' sense that applies to the other entries in the table. On average it monitors targets continuously for about $6 \mathrm{~h}$ per night. Additionally assuming operations for $5 \mathrm{yr}$ with $35 \%$ weather losses, this yields a total monitoring time of about $300 \mathrm{~d}$, which is the effective baseline used for computing the detection limit in Fig. 9.

detection thresholds of several current and future transit surveys that target WDs, whose properties are listed in Table $4 .{ }^{9}$ This reveals that the sensitivity to transits of small objects around individual WDs will improve substantially in the near future. However, the number of WDs that can/will be targeted by the upcoming missions (rightmost column in Table 4) is fairly limited compared with the reciprocal of the typical transit probability $\left(1 / p_{\text {tr }} \sim 100\right)$. Therefore, if small WD planets are rare, their detection via transits will likely remain elusive, even though the constraints on their occurrence rate will improve. The low transit probability can in principle be countered by preferentially targeting systems known to be oriented edge-on, for example from the detection of circumstellar-gas absorption (Gänsicke et al. 2012; Xu et al. 2016), but there are currently only a couple of WDs with such inclination constraints.

Other techniques of detecting exoplanets may be considered, but most have some drawbacks compared with the transit method:

(i) Detecting low-mass planets around WDs using the radialvelocity method is generally not feasible, because there are so few strong absorption lines in WD spectra and they are very broad.

(ii) Substellar companions such as brown dwarfs can be detected around WDs from the contribution of their IR emission to the system's spectral energy distribution (e.g., Farihi et al. 2008b), although it can be difficult to distinguish between a companion and circumstellar dust using the IR photometry alone. Sandhaus et al. (2016) have investigated the potential of this method for discovering small, non-transiting planets, but find that it is generally sensitive only to bodies with radii larger than about $2 \mathrm{R}_{\oplus}$.

(iii) Smaller bodies (possibly down to $100-\mathrm{km}$-sized asteroids)

9 The detection limits shown in Fig. 9 are for planets orbiting at $r_{2: 1}$. Planets orbiting at $r_{\mathrm{R}}$ transit twice more frequently than those at $r_{2: 1}$, but with transit durations that are a factor $2^{1 / 3}$ shorter. Both these differences affect $N_{\text {in }}$, so the detection limits for bodies at $r_{\mathrm{R}}$ should be lower by a factor $\sqrt{2 / 2^{1 / 3}}=2^{1 / 3} \approx 1.26$ compared with the dashed lines in the figure. may be detected by considering the IR variability caused by their hot dayside coming in and out of view (Lin \& Loeb 2014). For cool ( $\left.T_{\text {eff }} \lesssim 10,000 \mathrm{~K}\right)$ WDs, however, this technique likely requires lengthy space-based observations (e.g., using JWST), because of the low temperatures expected for rocky bodies located just outside the Roche limit.

(iv) Close-in planets around magnetic WDs may produce detectable electron-cyclotron maser emission through their interaction with the WD's magnetic field ( $\mathrm{Li}$ et al. 1998; Willes \& Wu 2004, 2005). ${ }^{10}$ Magnetic WDs, however, only comprise a small fraction of all isolated WDs (2 to 20\%; Ferrario et al. 2015).

\subsection{Possible application to WD $1145+017$}

The discovery of transits in the light curve of WD 1145+017 (Vanderburg et al. 2015) has opened up a new window onto the circumstellar environment of polluted WDs. A wealth of observational data is now available for this star, but the comprehensive picture of what ultimately causes the transits remains unclear. Here, we briefly review the relevant observations of WD 1145+017 and discuss whether its curious properties can be explained by the model of second-generation planet formation presented in this paper.

\subsubsection{Observational clues and their interpretation}

WD $1145+017$ is a heavily polluted helium-atmosphere WD whose light curve shows a multitude of recurring transit features (Vanderburg et al. 2015). The dips are deep (up to 60\%), long ( $\gtrsim$ several minutes), irregular in shape, and occur at multiple longitudes along the orbit, sometimes collectively covering a large fraction of the orbital cycle. Their recurrence periods fall between about 4.5 and $5 \mathrm{~h}$, corresponding to Keplerian orbital periods at and/or near the Roche limit (see Eq. (2)). The shapes and depths of the dips evolve on timescales of days and individual transit features seem to gradually appear and disappear over the course of several weeks or months (Gänsicke et al. 2016; Rappaport et al. 2016; Gary et al. 2017).

In addition to its peculiar light curve, WD 1145+017 also exhibits excess IR emission from circumstellar dust (Vanderburg et al. 2015; Xu et al. 2018) and evolving spectral absorption features due to metallic circumstellar gas (Xu et al. 2016; Redfield et al. 2017). The WD itself has an effective temperature of $15,900 \pm 500 \mathrm{~K}$, corresponding to a cooling age of $175 \pm 75 \mathrm{Myr}$ (Vanderburg et al. 2015). Its atmospheric metal pollution constitutes about $6.6 \times 10^{23} \mathrm{~g}$ of material (almost the mass of Ceres), yielding an estimated steady-state mass accretion rate of $4.3 \times 10^{10} \mathrm{~g} \mathrm{~s}^{-1}$ over sinking timescales of 0.3 to $0.6 \mathrm{Myr}$ (Xu et al. 2016).

The properties of the dips in the light curve of WD $1145+017$ are inconsistent with transits of solid-body planets. Instead, the irregular, slowly evolving shapes and the transient nature of the dips suggest that they are caused by transiting clouds of dust (Vanderburg et al. 2015). However, while individual transit features come and go, the most notable periodicity associated with the transits (about $4.5 \mathrm{~h}$ ) has remained present in the light curve over a timespan of several years (e.g., Gary et al. 2017). Furthermore, the periods of some transit features were found to remain stable to within

10 Incidentally, the 10-h-period terrestrial planet proposed by $\mathrm{Li}$ et al. (1998) to explain the peculiar emission lines of the highly magnetic WD GD 356 (see also Wickramasinghe et al. 2010) could have formed via the exoplanet-recycling scenario presented in this paper. 
20 ppm over several months (Rappaport et al. 2018). These properties seem to be incompatible with dust clouds, which are expected to disperse due to Keplerian shear within a few orbits.

The long-term stability of the transit recurrence periods can be explained by a scenario in which the dust that causes the dips is released quasi-continuously from massive bodies that carry enough angular momentum to have highly stable orbital periods (Vanderburg et al. 2015). ${ }^{11}$ The release of short-lived dust from these muchlonger-lived objects may happen either through their direct disintegration (Vanderburg et al. 2015) or via cratering erosion of larger bodies by somewhat smaller ones (Gary et al. 2017). Tidaldisruption simulations show that an internally differentiated body orbiting at the Roche limit may indeed be able to sustain prolonged intermittent mass loss (Veras et al. 2017).

Assuming the dust that causes the dips is indeed released from massive bodies, the observed pattern of transit periods may provide insight into the orbital configuration and nature of these objects. In the original K2 light curve (from which the peculiar nature of WD 1145+017 was discovered), six periodicities could be discerned, ranging from 4.499 to $4.858 \mathrm{~h}$ (Vanderburg et al. 2015). The two most prominent of these, at 4.499 and $4.605 \mathrm{~h}$, have since been recovered in ground-based data that was taken several years later (Rappaport et al. 2016; Gary et al. 2017), suggesting that at least these two periods correspond to massive objects and lending some credibility to the other four. Most of the dips seen in the groundbased light curves, however, are associated with short-lived features and repeat at slightly (but statistically significantly) shorter periods, mostly between 4.490 and $4.495 \mathrm{~h}$ (Gänsicke et al. 2016; Rappaport et al. 2016, 2018; Croll et al. 2017; Gary et al. 2017).

To explain the observed pattern of transit periods, Rappaport et al. (2016) suggest that the 4.499-h period corresponds to a longlived, massive body that fills its Roche lobe, while the 4.490-to4.495-h periods correspond to short-lived fragments of this body, released via its L1 Lagrange point onto slightly smaller orbits. In this scenario, the observed ratio of transit periods can be used to estimate the mass of the parent body, for which Rappaport et al. (2016) find $10^{23} \mathrm{~g}$ (about 0.1 Ceres mass). Also regarding this scenario specifically, Gurri et al. (2017) find that the parent body cannot be more massive than $10^{23} \mathrm{~g}$, else its perturbations of the fragments would cause period deviations violating the observational upper limits on their period stability (see also Veras et al. 2016b).

\subsubsection{The origin of massive bodies just outside the Roche limit}

It is clear that many of the properties of WD 1145+017's transits can be explained if the dust causing the dips is released from longlived, massive objects. An important unresolved issue for this scenario, however, is how these bodies came to be in 4.5-to-5-h-period orbits, which lie just outside the Roche limit, far inside the region that was cleared during earlier stages of the star's evolution. As an additional complication, the putative bodies likely need to be on low-eccentricity $(\lessgtr 0.1)$ orbits to avoid immediate tidal disruption, because the efficiency of this process quickly increases with decreasing pericentre distance (Veras et al. 2017).

11 An alternative explanation for the period stability of the transits is that the transiting clouds of dust are trapped in the WD's magnetosphere and therefore corotate with the WD (Farihi et al. 2017). The magnetic field strength required for this scenario, however, seems to be far above the observational upper limits for WD 1145+017 (Farihi et al. 2018; Rappaport et al. 2018).
Gänsicke et al. (2016) have suggested that the close-in bodies may be the result of the tidal circularisation of a (minor) planet that was previously on a highly eccentric orbit with its pericentre close to the Roche limit (see also Sect. 1.2 of Veras \& Gänsicke 2015). A potential problem with this scenario is that, because of angularmomentum conservation, the radius of the circularised orbit would come to be a factor $\sqrt{2}$ larger than the original orbit's pericentre distance. Since the latter would have to be outside the Roche limit to avoid immediate tidal disruption, it is difficult to intactly place a massive body on an orbit very close to the Roche limit $\left(r / r_{\mathrm{R}}<\sqrt{2}\right)$ through tidal circularisation. Radiative forces, such as PR drag or the Yarkovsky effect, can remove angular momentum, circularising orbits without increasing their pericentre distance, but over the lifetime of a WD this only works for smaller objects (i.e., dust, pebbles, and boulders; Veras et al. 2015). Alternatively, the circumstellar gas disc detected around WD 1145+017 may have helped in the circularisation process (Gänsicke et al. 2016). It is still unclear, however, whether enough gas is available around WD $1145+017$ for this to work and, if so, whether it can dissipate energy fast enough.

In short, it is challenging to explain the proposed massive bodies by invoking the migration of intact planetesimals from higheccentricity, AU-scale orbits to low-eccentricity, 4.5-to-5-h-period ones. In contrast, the recycling mechanism studied in this paper gives a natural explanation for massive bodies on circular orbits close to the Roche limit. In the region just outside the Roche limit, it predicts a large number of small bodies that orbit close to one another, which may explain the observed multitude of transit periods. Furthermore, it allows the formation of minor planets as massive as 0.1 Ceres mass (as suggested by Rappaport et al. 2016) within the 175-Myr age of the system (see Figs. 6-8), although the occurrence of such massive objects very close to the Roche limit requires a very massive disc (e.g., the top-right panel of Fig. 8 shows $10^{23}$-g bodies at $\Delta \lesssim 0.01$ ), unless the objects can somehow migrate inwards after being assembled further out (for instance through interaction with the gaseous disc). A remnant of the massive debris disc that produced the bodies could either still be present, or it may since have been destroyed (see Sects. 4.2 and 4.5.6).

\subsubsection{Ongoing planet formation in the WD 1145+017 system?}

In the scenario sketched above, the dust clouds that cause the dips in the light curve of WD $1145+017$ are emitted by pre-existing massive bodies that are gradually destroyed. Our study brings forward another possibility: the dust clouds may be related to the ongoing formation of massive bodies from a disc that is currently overflowing the Roche limit. In this process of second-generation planet formation (one of the main topics of this paper), numerous collisions occur between rocky building blocks. So far, we have treated these collisions as idealised instantaneous perfect mergers, but in reality many of them likely cause at least some initial partial disruption of the bodies involved, after which the resulting debris gradually reagglomerates (cf. Hyodo \& Ohtsuki 2015; Hyodo \& Charnoz 2017). The dust clouds transiting WD 1145+017 may be manifestations of such disruptive collisions.

In this scenario, the most prominent cluster of transit periods seen in the light curve (around $4.5 \mathrm{~h}$ ) could be associated with the outer edge of the disc (at the Roche limit) and/or the region immediately beyond it, where frequent collisions occur between newly formed bodies. Dips that repeat at somewhat longer periods could then be due to collisions involving bodies that formed some time ago and have since migrated some distance away from the disc. Figure 10 gives an overview of the WD $1145+017$ system in this 
scenario, specifically assuming that the Roche limit corresponds to an orbital period of $P_{\mathrm{R}}=4.490 \mathrm{~h}$ (i.e., the lower end of the observed range of transit periods). In the following sections, we discuss this model in more detail.

\subsubsection{The transit periods around $4.5 \mathrm{~h}$}

With the Roche limit at an orbital period of $P_{\mathrm{R}}=4.490 \mathrm{~h}$, as we have assumed for Fig. 10, the transit periods close to $4.490 \mathrm{~h}$ may correspond to dust clouds generated in the continuous and/or discrete regimes of planet formation (see Sect. 2.4.4). If this is the case, the days and weeks-to-months timescales on which these dips seem to vary (see, e.g., Figs. 3 and 4 of Rappaport et al. 2018) could be related to the timescale on which new bodies gradually form (see Eqs. (34) and (35)). Specifically regarding the formation of discrete-regime bodies, collisions (of continuous-regime building blocks onto a forming discrete-regime body) are expected to gradually become less frequent as the body grows and moves away from the Roche limit, because this allows the continuous-regime building blocks to build up to a larger size before they merge with the discrete-regime body (see also Fig. S1 of CC12). This cycle would then repeat once the formation of one discrete-regime body is complete and the production of the next one starts - i.e., on a timescale of $t_{\mathrm{d}} \approx 1.5$ months.

On the other hand, for plausible values of $\mathcal{T}$ (see Eq. (30)), the continuous and discrete regimes end very close to the Roche limit $\left(\Delta_{\mathrm{d}} r_{\mathrm{R}} \lesssim 100 \mathrm{~km}\right.$ for $\left.\mathcal{T} \gtrsim 10^{9}\right)$. At such small distance scales, certain implicit assumptions of our model may start to break down. Firstly, our model assumes the Roche limit is a sharp boundary. However, variations in material density $\rho_{\mathrm{d}}$ of the order of $1 \%$, for example, will move its location by a few thousand km (see Eq. (1)). Secondly, the vertical size of the dust clouds orbiting WD 1145+017 must be comparable to the size of the WD (in order to account for transit depths of $60 \%$ ). If their radial extent is similar to this, then clouds on orbits with periods between 4.490 and $4.499 \mathrm{~h}$ will overlap, so they may interact in ways not accounted for. Finally, the dispersion of transit periods around $4.5 \mathrm{~h}$ may also be due to the effects of radiation pressure, which can affect the orbital periods of (clouds of) dust grains significantly. ${ }^{12}$ This all goes to say that our simple analytical model (Sect. 2.4) may not include all the physics necessary to fully understand the cluster of periodicities around $4.5 \mathrm{~h}$.

\subsubsection{Minor planets at longer periods}

The middle panel of Fig. 10 shows what our model means quantitatively for the longer periodicities seen in the K2 data. Because the disc needs to be younger than the age of the system (175 Myr), rough lower limits can be put on the masses of the bodies that presumably orbit at these periods. Our numerical simulations show that discs below this age generally do not produce minor planets with masses far below the purple $\mathcal{T}=10^{15}$ line (see Fig. 8). In

12 For example, spherical grey dust grains of size $s=3 \mu \mathrm{m}$ (well above the minimum grain size derived from multiwavelength transit observations; $\mathrm{Xu}$ et al. 2018) and density $\rho_{\mathrm{d}}=3 \mathrm{~g} \mathrm{~cm}^{-3}$ in orbit around WD $1145+017$ (with parameters $M_{\star} \approx 0.6 \mathrm{M}_{\odot}, L_{\star} \approx 0.009 \mathrm{~L}_{\odot}$; Vanderburg et al. 2015) would have a radiation-pressure-to-gravity force ratio of $\beta \approx 0.001$ (e.g., Burns et al. 1979). After being released from a massive $(\beta \simeq 0)$ body whose orbit is circular, such grains would orbit the WD at a period that is a factor $(1-\beta) /(1-2 \beta)^{3 / 2} \simeq 1+2 \beta \approx 1.002$ longer than that of their parent body (e.g., Eq. (B6) of Rappaport et al. 2014), which is comparable to the ratio of the observed periods $(4.499 \mathrm{~h} / 4.490 \mathrm{~h} \approx 1.002)$.
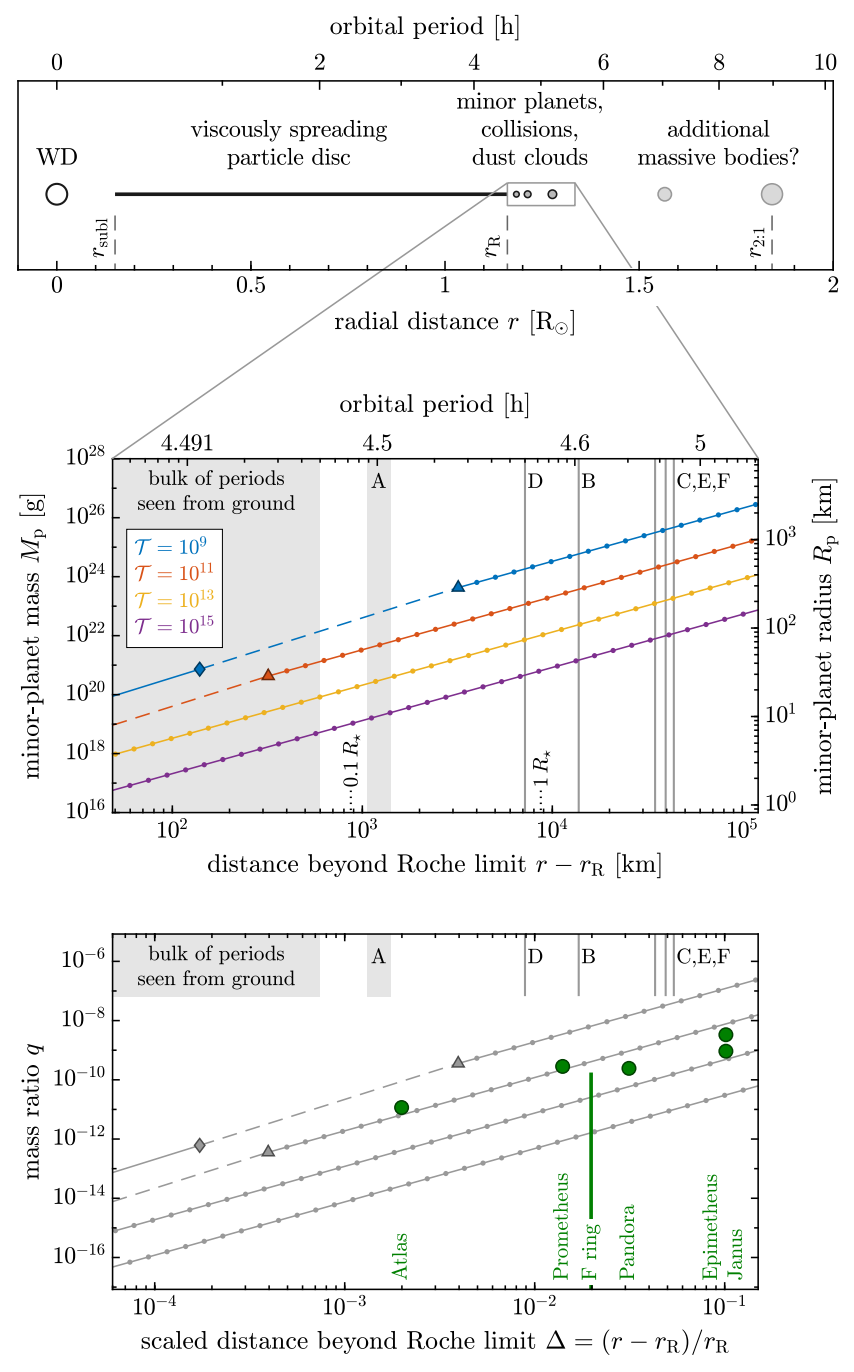

Figure 10. Overview of a possible model for the WD 1145+017 system, which assumes that the main transit period seen in the star's light curve corresponds to the outer edge of a viscously spreading debris disc, located at the Roche limit (i.e., $P_{\mathrm{R}}=4.490 \mathrm{~h}$ ). In addition, we have assumed a WD mass of $M_{\star}=0.6 \mathrm{M}_{\odot}$ and a Roche prefactor of $C_{\mathrm{R}}=2.0$. Top panel: Cartoon showing a radial cross-section of the system. Key radial distances are marked: $r_{\text {subl }}$, the sublimation radius; $r_{\mathrm{R}}$, the Roche limit; $r_{2: 1}$, the radius for which the 2:1 MMR coincides with the Roche limit. The WD and the planetary bodies are not drawn to scale. Middle panel: Log-scale zoom of the region just outside the Roche limit, showing the observed transit periods (vertical grey bands and lines) along with analytical minor-planet mass-distance relations (coloured lines and symbols). Labels 'A' through ' $F$ ' mark the periodicities seen in the $\mathrm{K} 2$ data, in order of their signal amplitude (Vanderburg et al. 2015). The various colours, line-styles, and symbols used in the analytical mass-distance relations have the same meaning as in Fig. 5 (see caption of that figure). Also indicated (along the bottom axis) are distances equalling the WD's radius and one tenth of that, below which the radial extent of the transiting dust clouds may be greater than the radial separation of adjacent orbits. Bottom panel: Same as the middle panel, but in dimensionless parameters. This allows a comparison with the Saturnian system, which is shown in green. The properties of Saturn's moons were taken from JPL's Solar System Dynamics website (http://ssd.jpl.nasa.gov). The location of Saturn's F ring comes from Bosh et al. (2002); its mass (though poorly constrained) is probably not greater than that of its two shepherding moons (see Hyodo \& Ohtsuki 2015). We set the Roche limit for Saturn to be at $r_{\mathrm{R}}=137,500 \mathrm{~km}$, (i.e., in between the outer edge of Saturn's A ring and Atlas, the first satellite outside the main rings). 
addition, the analytical mass-distance relations with a high normalisation (i.e., low $\mathcal{T}$ ) require a recently created and/or massive disc. Given the low likelihood of witnessing an $M_{\text {disc }} \gtrsim 10^{28} \mathrm{~g}$ disc within $1000 \mathrm{yr}$ of its formation, minor planets with masses above the blue $\mathcal{T}=10^{9}$ line are improbable (see Fig. 8).

Combining the above two considerations yields rough constraints on the masses of the bodies corresponding to the various observed transit periods. The ' $\mathrm{B}$ ' period at $4.605 \mathrm{~h}$, for example, would likely correspond to a body of mass $10^{21} \mathrm{~g} \lesssim M_{\mathrm{p}} \lesssim 10^{25} \mathrm{~g}$ (i.e., a radius between a few tens and several hundreds of $\mathrm{km}$ ), with masses towards the lower end of this range being more likely. If the presence of massive bodies around WD $1145+017$ is confirmed, this hypothesis of their origin could be tested by estimating the masses of the bodies. Most stringently, their relative masses as a function of their separation from the Roche limit should roughly follow the pyramidal-regime mass-distance relation $\left(M_{\mathrm{p}} \propto \Delta^{9 / 5}\right)$.

Additional massive bodies may be present around WD 1145+017, including more massive ones at longer orbital periods. In particular, there may be a large planet orbiting at $r_{2: 1}$ (i.e., with an orbital period of $P_{2: 1} \approx 9 \mathrm{~h}$ ), as seen in many of the numerical simulations (see Figs. 7, top-right panel, and 8). This planet could have evaded detection so far because it does not transit the WD and does not emit dust like the bodies responsible for the observed transits. Our model, however, does not require the presence of any additional bodies. The disc's Roche-limit overflow may have started relatively recently, such that the pyramidal regime has not yet been filled (see, e.g., the cyan circles for $t=10^{7} \mathrm{yr}$ in the bottom-right panel of Fig. 8).

Also shown in Fig. 10 (in the bottom panel) is a comparison of WD 1145+017 with the Saturnian system. This shows that (when expressed in dimensionless distance $\Delta$ ) the positioning and spacing of WD 1145+017's transit periods bears some resemblance to that of Saturn's small inner moons, which are likely to have formed out of the planet's rings (Charnoz et al. 2010, 2011; CC12). Saturn's rings are not currently overflowing the Roche limit (being held back temporarily by satellite MMR torques; Tajeddine et al. 2017), explaining why this system at present does not show the frequent, disruptive, dust-generating collisions that we infer to be associated with the planet-formation process around WD 1145+017. Nevertheless, the presence of Saturn's F ring (likely the result of a relatively recent collision between two small moons; Hyodo \& Ohtsuki 2015) shows that violent collisions between building blocks are a regular occurrence in these systems. At times of active satellite formation, collisions are likely to be much more frequent.

Figure 10 also shows that the spacing of objects in both systems is markedly wider than the predictions of the analytical model (Eq. (37); shown by the dots in the mass-distance relations). For WD 1145+017, it could be that additional bodies are present but remain undetected because they have not recently undergone dustproducing collisions. If this is the case, continued monitoring of the WD should reveal transits at periods between the observed ones. Alternatively, our analytical model may underestimate the spacing of the bodies, because it does not take into account that gravitational interactions between the massive bodies may increase the average spacing at which mergers happen (currently set to two mutual Hill radii in the model; see Sect. 2.4.5). Future modelling efforts could include N-body simulations to resolve this issue. Alternatively, the merger distance in the model could simply be increased to match the Saturn data, or it could be replaced with a prescription derived from stability-analysis studies (e.g., Chambers et al. 1996).

The more general pyramidal-regime prediction of $\mathrm{d} n / \mathrm{d} \Delta \propto$ $1 / \Delta$ does seem to hold approximately for the Saturnian moons (i.e., they are roughly equidistant in $\log (\Delta)$, especially when considering the Epimetheus-Janus pair as a single object; see also Fig. 1b of CC12). For WD 1145+017, the distribution of transit periods found in the K2 data may be closer to equidistant in $\Delta$ (see also Fig. 1a of Vanderburg et al. 2015), but most of these periodicities still need to be confirmed. Given the difficulty of measuring the masses of very small bodies around a WD, an accurate census of the periodicities in WD 1145+017's light curve may be the most accessible way of testing our model.

\subsubsection{Implications for IR excess and mass accretion rate}

If WD $1145+017$ currently hosts a massive debris disc that generates (minor) planets at its outer edge (as postulated in Sect. 4.5.3), then this disc will cause IR emission and metal accretion onto the WD. Here, we discuss these implications in the context of observational constraints.

The observed IR excess of WD $1145+017$ constitutes a fractional luminosity of about 0.3 to $0.5 \%$ and requires a projected area of dust that is roughly 50 to 250 times the cross-section of the stellar disc (Vanderburg et al. 2015; Zhou et al. 2016; Xu et al. 2018). The postulated massive debris disc would produce a significant amount of IR emission (by reprocessing stellar radiation), but, since the disc must be oriented close to edge-on to produce bodies at (nearly) transiting inclinations, we would only detect a small fraction of its emission. Vanderburg et al. (2015) find that a flat, optically thick disc with an inclination aligned with the transiting bodies produces less IR emission than the observed excess. Therefore, the existing photometric data are consistent with the presence of an edge-on massive disc. Fully explaining the observed excess, however, also requires an additional dust population with a different configuration. One possibility is that the IR excess is mostly due to the dust released from the massive bodies. The dust clouds seen in transit already account for some 10 to $20 \%$ of the emitting surface area inferred from the IR excess (Xu et al. 2018) and it is likely that there is more dust associated with these clouds that passes above or below the stellar disc from our viewpoint.

The mass accretion rate of WD $1145+017$ inferred from its atmospheric pollution $\left(4.3 \times 10^{10} \mathrm{~g} \mathrm{~s}^{-1}\right.$; Xu et al. 2016) is relatively high (although, unlike the transits, this is not unique to this WD; see, e.g., Dufour et al. 2012). PR drag (on a physically thin, flat disc) can only explain accretion rates up to $\sim 10^{8} \mathrm{~g} \mathrm{~s}^{-1}$ (see Eq. (15); Rafikov 2011b), so an additional effect must be at play. We find that viscous spreading can yield the observed accretion rate for discs with masses of a few times $10^{27} \mathrm{~g}$ (see Fig. 7, bottom-right panel). Hence, if the debris disc that we propose for WD $1145+017$ is massive enough, it can potentially explain two uncommon properties of this WD: transits with stable periods and a high accretion rate.

Finally, the presence of a disc that overflows the Roche limit relaxes the requirement that the mass flow inferred from the transits ( 10 $0^{11} \mathrm{~g} \mathrm{~s}^{-1}$; Gänsicke et al. 2016; Rappaport et al. 2016) must equal that derived from the atmospheric pollution, because the system is not in steady state in this case. Instead, the disc constitutes a large mass reservoir, from which mass flows both inwards (explaining the mass accretion onto the WD) and outwards (producing the massive bodies associated with the transits).

\subsection{Exoplanet recycling around non-WD hosts}

While our study is primarily concerned with the formation of second-generation planets around WDs, the recycling scenario 
could in principle also operate around other astrophysical objects, as long as they are dense enough for the Roche limit (for appropriate material densities) to lie far enough outside their surface (see Eq. (1)). Here, we briefly discuss a few interesting cases, noting that further study would be needed to evaluate the planet-forming potential of compact particle discs in these different settings in more detail.

\subsubsection{Neutron stars}

Because neutron stars are even more compact than WDs, the space between their surface and the Roche limit, where a tidal disc of particles may be located, is substantial. Incidentally, low-mass $\left(M_{\mathrm{p}} \ll 1 \mathrm{M}_{\text {Jup }}\right)$ planets can be detected efficiently around pulsars (highly magnetised, rapidly rotating neutron stars that emit collimated beams of electromagnetic radiation) by searching for planetary perturbations on the otherwise highly stable timing of their pulsed emission (e.g., Cordes 1993). Using this method, two superEarth-mass planets and one Moon-mass planet were inferred to orbit the pulsar PSR B1257+12 on low-eccentricity orbits with periods between 25 and 100 d (Wolszczan \& Frail 1992; Wolszczan 1994; Konacki \& Wolszczan 2003). The orbital periods of these planets are much longer than those predicted for the recycled planets in this paper (up to $\sim 10 \mathrm{~h}$; see Eq. (46)), indicating that the recycling mechanism as presented here is not responsible for them. We note, however, that one of the suggested formation scenarios for the pulsar planets involves the tidal disruption of a companion star (e.g., Phinney \& Hansen 1993; Currie \& Hansen 2007; Margalit \& Metzger 2017), bearing some resemblance to the recycling scenario presented in this paper.

Despite the high sensitivity to planets (e.g., Thorsett \& Phillips 1992), the trio of planets around PSR B $1257+12$ remains the only confidently detected set of low-mass planets orbiting a neutron star. Possible reasons why the recycling mechanism presented in this paper does not (often) produce planets around neutron stars are as follows. (1) Neutron-star systems with planetary material available to form a debris disc may be rare, because the planets that orbit a neutron-star progenitor are easily lost during the supernova in which the neutron star is created. Asymmetric supernovae may kick neutron stars away from their planetary systems and, even in symmetric supernovae, the sudden mass loss involved can place planets on unbound hyperbolic trajectories (Thorsett \& Dewey 1993). (2) The sublimation radius around a neutron star may lie outside the Roche limit, precluding the formation of a rocky debris disc. The location of the sublimation radius depends on what fraction of the star's spin-down luminosity is available for the heating of dust and debris, which is not well understood. Estimates by Wang et al. (2006) and Shannon et al. (2013) place the sublimation radius for rocky material beyond the Roche limit.

\subsubsection{Hot subdwarfs}

Hot subdwarfs are core-helium-burning stars at or beyond the blue end of the horizontal branch, thought to originate from red-giantbranch stars that undergo enhanced mass loss (possibly due to interaction with a close binary companion) or from mergers of lowmass-WD pairs (Heber 2009, 2016). With typical masses close to $0.5 \mathrm{M}_{\odot}$ and radii of around $0.2 \mathrm{R}_{\odot}$ (e.g., Van Grootel et al. 2010; Fontaine et al. 2012), the Roche limit is well outside the stellar surface for these stars. However, hot subdwarfs have effective temperatures exceeding $20,000 \mathrm{~K}$, which may complicate the survival of rocky debris inside the Roche limit against sublimation.
Two B-type hot subdwarfs have been reported to host closein, Earth-sized candidate exoplanets: Kepler-70, a 2-planet system (Charpinet et al. 2011), and KIC 10001893, a 3-planet system (Silvotti et al. 2014). ${ }^{13}$ In both cases, the claimed planets have orbital periods of 5 to $20 \mathrm{~h}$, with period ratios close to MMRs. The suggested explanation for the origin of such planets is the engulfment of some of a star's original gas-giant planets during its red-giantbranch evolution, after which the planets undergo rapid inward migration and extensive mass loss, while the star is stripped of much of its envelop and becomes a hot subdwarf (Charpinet et al. 2011; see also Passy et al. 2012). In a variation of this formation scenario, only a single giant planet is engulfed and the observed planets are remnants of its tidally disrupted core (Bear \& Soker 2012).

Could the recycling mechanism presented in this paper play a role in the formation of the putative hot-subdwarf planets? On the one hand, their orbital periods are broadly consistent with the prediction of Eq. (46), which argues in favour of the recycling scenario. On the other hand, both reported systems feature multiple Earth-sized candidate exoplanets, contrary to the predicted architecture of the recycled planetary systems, which typically consist of a single large planet plus a set of much smaller ones. Our model, however, does not include resonant interactions between planets. Satellite-formation studies in which such effects are taken into account show that systems with two or three bodies of similar mass, orbiting in or close to MMRs with one another, can sometimes occur (Hyodo et al. 2015; Salmon \& Canup 2017). Finally, as mentioned above, the sublimation of rocky material inside the Roche limit may be a problem for the recycling scenario. However, circumstances may exist in which the debris is stable against sublimation (e.g., a high ambient gas pressure can quench sublimation; see also Rafikov \& Garmilla 2012). To sum up, the hot-subdwarf planets (if real) have the need to be generated after their host star's giant-branch phase and roughly fit the expected orbital periods, which is intriguing, but a more sophisticated model that can handle resonant interactions between planets is needed to understand whether they could have spawned from a massive tidal disc.

\subsubsection{Late-type main-sequence stars and substellar objects}

Most types of main-sequence stars have a relatively low mean density and a high luminosity, with the consequence that the Roche limit for such stars is inside the sublimation radius (or even inside the stellar radius for early-type stars). At the low-mass end of the main-sequence, however, the mean density goes up steeply (reaching a maximum of around $\rho_{\star} \sim 10^{2} \mathrm{~g} \mathrm{~cm}^{-3}$ at the star/brown-dwarf boundary), while luminosity goes down. As a result, late M-type main-sequence stars, as well as substellar objects such as brown dwarfs and giant planets, can host rocky debris discs in the space between their surface or the sublimation radius and the Roche limit. For most of these central objects, however, this space is not available immediately (i.e., while first-generation planets may be forming), because they take a long time to contract to their eventual compact size (see Fig. 11). Hence, any possible tidal discs around brown dwarfs and M dwarfs would have to form later (after several

\footnotetext{
13 The evidence for the hot-subdwarf planets - sinusoidal modulations in the light curves of the stars, interpreted as the daysides of planets coming in and out of view (Charpinet et al. 2011; Silvotti et al. 2014) - is far from conclusive. Krzesinski (2015), for instance, has challenged the planetary origin of the light-curve modulations.
} 


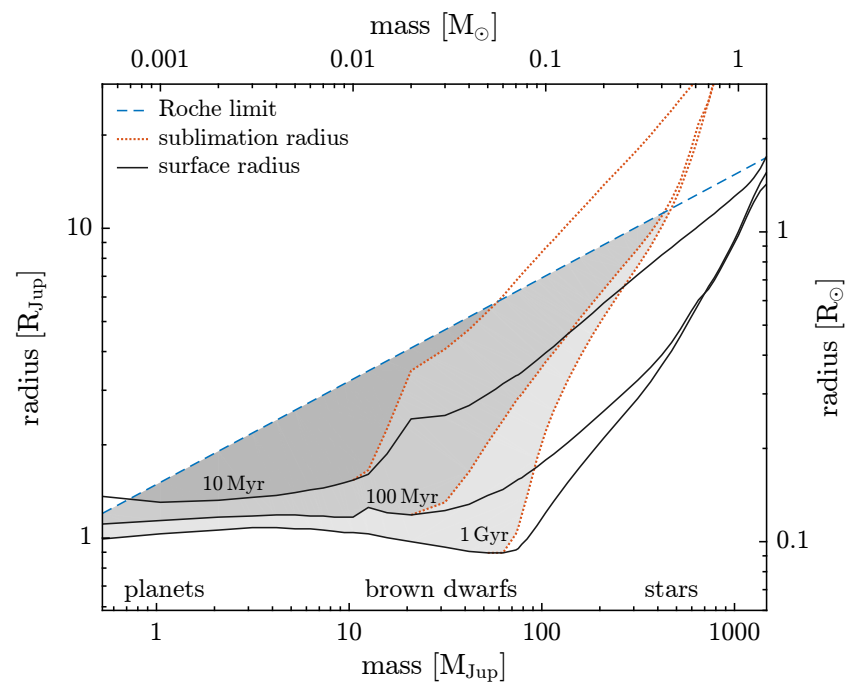

Figure 11. Mass-radius relations for low-mass stars and substellar objects at three different ages (black solid lines), together with the associated sublimation distances (red dotted lines), compared with the Roche limit (blue dashed line). Shaded areas mark the region where a tidal disc made of rocky debris can exist. The mass-radius relations combine results from Baraffe et al. (1998, brown dwarfs and stars) and Baraffe et al. (2003, planets and brown dwarfs), with the transition between the two models at the (timedependent) point where the central object has an effective temperature of $2600 \mathrm{~K}$. The Roche limit is calculated from Eq. (1), assuming a material density of $\rho_{\mathrm{d}}=3 \mathrm{~g} \mathrm{~cm}^{-3}$ and a Roche prefactor of $C_{\mathrm{R}}=2.0$. For the sublimation radius, we use the radial distance at which grey dust reaches a temperature of $1500 \mathrm{~K}$, using central-object temperatures given by the model isochrones.

tens of Myr), for instance when a late instability in the host's planetary system sends a massive body into the tidal disruption sphere.

If compact tidal discs do occur around mature low-mass stars or substellar objects, and if these discs are massive enough, they may produce second-generation (minor) planets via the recycling mechanism. A complication may be that planet migration due to stellar tides (which we neglected in our model for WDs) may have to be taken into account for these hosts, because they are not as compact as WDs. If the central object's rotation period is longer than $P_{2: 1}$, the tidal migration of planets at $r \leq r_{2: 1}$ is directed inwards. Mature brown dwarfs mostly have rotation periods of the order of a few hours (e.g., Radigan et al. 2014; Metchev et al. 2015), so tidal inspiral is unlikely to pose a problem for recycled planets around most of them. The rotation periods of old M dwarfs, however, often reach values of the order of 1 to $100 \mathrm{~d}$ (e.g., Irwin et al. 2011). Therefore, if the tidal torques are strong enough, they may prevent recycled planets around $\mathrm{M}$ dwarfs from persisting after the disc's mass decreases (see also Rosenblatt et al. 2016).

Do we know of any exoplanets that could have formed in a recycling event? Not many exoplanets are known that orbit late $\mathrm{M}$ dwarfs and none are presently known around brown dwarfs (He et al. 2017). Most of the low-mass planets discovered around late M dwarfs (e.g., GJ 1132 b, Berta-Thompson et al. 2015; Proxima Centauri b, Anglada-Escudé et al. 2016; the TRAPPIST-1 planets, Gillon et al. 2016; YZ Ceti bcd, Astudillo-Defru et al. 2017; LHS 1140 b, Dittmann et al. 2017) have orbital periods that are significantly longer than the prediction of Eq. (46). An exception is Kepler-42 c, which has an orbital period of $10.8 \mathrm{~h}$ (Muirhead et al. 2012). The fact that this planet is situated in a multi-planet system with similarly sized planets further out, however, suggests a more conventional (first-generation) formation scenario.

In short, none of the currently known exoplanets around lowmass stars seem to have formed via the recycling scenario. However, late $\mathrm{M}$ dwarfs and brown dwarfs have not yet been surveyed for exoplanets very extensively. It is possible that recycled exoplanets do occur around these objects, but have not yet been found. Ongoing and future exoplanet-detection programs specifically targeting late $\mathrm{M}$ dwarfs and/or brown dwarfs (e.g., MEarth, Nutzman \& Charbonneau 2008; SPECULOOS, Burdanov et al. 2017) may be able to determine whether recycled exoplanets occur around these hosts and, if so, how common they are.

\section{CONCLUSIONS}

This paper considered the evolution of massive debris discs around WDs, using both analytical and numerical techniques. Following previous work on WD debris discs, we start by assuming that these discs are located inside the star's tidal disruption zone and that they are geometrically and dynamically similar to Saturn's rings. As a first conclusion, we reiterate the result known for planetary rings (e.g., Daisaka et al. 2001) that vertically optically thick discs located in the outer parts of the tidal disruption zone are gravitationally unstable (see Eqs. (10) and (11); Fig. 2), which means that they have an enhanced effective viscosity due to self-gravity wakes.

Previous theoretical work on the evolution of WD debris discs has focussed on disc evolution caused by PR drag (Rafikov 2011b; Bochkarev \& Rafikov 2011). Our findings for low-mass discs are consistent with the results of these studies (e.g., Fig. 6, top-left panel; Fig. 7, bottom panels), but we also quantify the upper limit in surface mass density for which they are valid. For values above $10^{3}$ to $10^{4} \mathrm{~g} \mathrm{~cm}^{-2}$ (corresponding to disc masses around the mass of Pluto; $\sim 10^{25} \mathrm{~g}$ ), viscous spreading becomes more important than PR drag for the disc's evolution (see Eqs. (17) and (18); Fig. 3).

The effective viscosity of a gravitationally unstable particle disc increases rapidly with distance (see Eq. (13)). As a result, discs whose evolution is dominated by viscous spreading have an outer edge that moves outwards rapidly, while a pile-up of material forms at their inner edge, which only moves inwards slowly. For intermediate-mass discs (with masses from $10^{25}$ to $10^{26} \mathrm{~g}$, depending on their radial location), the viscous spreading can decrease surface densities to the point where PR drag takes over and subsequent evolution follows that known for low-mass discs (e.g., Fig. 6, top-right panel). For discs more massive than about $10^{26} \mathrm{~g}$ (roughly the mass of Io), however, the outer edge of the disc has reached the Roche limit before this happens (see Eq. (20); Fig. 4).

When disc material spreads to beyond the Roche limit, it is no longer prevented from coagulating by tidal forces and Keplerian shear, so this material will gather into larger bodies. Hence, viscous-spreading-dominated discs can produce a set of secondgeneration (minor) planets just outside their Roche limit (e.g., Fig. 6, bottom panel) in the same fashion that small moonlets are being formed at the outer edge of Saturn's rings (Charnoz et al. 2010). For massive discs, this process takes place on relatively short (sub-Myr) timescales (see Fig. 7, middle panels), while the discs themselves can survive against viscous spreading and PR drag for longer than the age of the universe (see Eq. (23); Fig. 7, bottom-left panel).

The bodies that form at the disc's outer edge migrate outwards by exchanging angular momentum with the disc via MMRs and they grow through mutual mergers. The interplay of these 
two effects results in a distinctive pattern of minor planets whose masses and mutual spacing increase with distance from the disc (see Eqs. (36) and (37); Fig. 5), as has previously been studied in detail for massive planetary-ring systems (CC12). At a given radial distance, minor-planet masses go down with time, since mass flows through to larger bodies at greater radial distances, while continued viscous spreading decreases the disc's surface density, reducing the Roche-limit mass outflow available for forming new bodies (see Figs. 6, bottom panel, and 8).

The outward migration of the bodies stops at $r_{2: 1}$, the radius at which their 2:1 MMR coincides with the Roche limit (because none of their first-order MMRs would overlap with the disc anymore if they were to migrate beyond this point). Only a limited amount of mass can be accommodated by minor planets in between the Roche limit and $r_{2: 1}$ (see Eq. (44)). Once this space has been filled up (which happens eventually for most planet-producing discs), material starts to accumulate into a single massive body at $r_{2: 1}$. This means that massive WD debris discs (i.e., with masses of about $10^{26} \mathrm{~g}$ and higher) generally give rise to a single massive planet orbiting at $r_{2: 1}$, together with a set of smaller bodies in smaller orbits (see Figs. 6, bottom panel, and 8).

The massive planet at $r_{2: 1}$ can take up a substantial fraction of the disc's original mass (tens of per cent; see Eq. (45); Fig. 7, top panels). This means that if a super Earth is tidally disrupted by a WD and forms a massive debris disc, it can be partially recycled to form an Earth-mass second-generation planet. Given its 2:1 MMR with the Roche limit, the planet at $r_{2: 1}$ has a clearly predictable orbital period (around $10 \mathrm{~h}$; see Eq. (46)). It is located in the WD's habitable zone for old, cool WDs (with cooling ages between about 2 and $10 \mathrm{Gyr}$; see Sect. 4.3). Constraints on the occurrence rate of this type of exoplanet are still relatively weak, but they are likely to be improved by several upcoming large, high-cadence transit surveys that include WD targets (see Sect. 4.4; Fig. 9; Table 4). The best place to look for such planets may be WDs that exhibit a strong IR excess and/or circumstellar gas in absorption, although it is also possible that a disc that gives rise to planets is destroyed after producing them (see Sect. 4.2).

As an application of our second-generation-planet-formation model, we have investigated whether it can shed light on the curious properties of WD $1145+017$ (Sect. 4.5). The light curve of this WD shows irregularly shaped dips, probably caused by transiting clouds of dust orbiting close to the Roche limit. These dips repeat with remarkably stable periods, which can be explained if the dust is released from long-lived, massive bodies. The presence of such bodies on low-eccentricity orbits close to the Roche limit, however, is difficult to explain, since this region has been cleared earlier in the star's evolution. Second-generation planet formation from a massive debris disc, as presented in this paper, provides a natural explanation for the presence of massive bodies just outside the Roche limit. Furthermore, if WD $1145+017$ currently hosts a disc that is overflowing the Roche limit, the transiting dust clouds may be the result of collisions that are part of the planet-formation process. In the context of this scenario, we make rough predictions for the masses of the putative bodies associated with the various observed transit periods (see Fig. 10).

While our study mainly focussed on what happens around the outer edge of massive WD debris discs, we also obtained results on the mass accretion rates of WDs that host such discs. Specifically, we find that in discs more massive than about $10^{27} \mathrm{~g}$ (i.e., the mass of a terrestrial planet) viscous spreading can directly dictate the inward mass flow at the sublimation radius, causing mass accretion rates of $\gtrsim 10^{9} \mathrm{~g} \mathrm{~s}^{-1}$ (see Fig. 7, bottom-right panel). Thus, the viscous spreading of very massive debris discs is a possible explanation for the high (super-PR-drag) mass accretion rates seen in some heavily polluted WDs. For WD $1145+017$, in particular, the presence of such a disc may be able to simultaneously explain the massive bodies related to its transits and the high mass accretion rate inferred from its atmospheric pollution.

Finally, we have explored the potential of other astrophysical objects to host tidal discs that can spawn second-generation planets (see Sect. 4.6). Objects with sufficient compactness include neutron stars and hot subdwarf stars, but in both cases sublimation may preclude the presence of rocky debris inside the Roche limit. In the case of neutron stars, the loss of planetary material during their birth supernovae may be an additional obstruction. A more likely class of hosts consists of late M-type main-sequence stars and substellar objects like brown dwarfs and giant planets. At a mature age, these objects are compact and cool enough to allow rocky debris to persist inside the Roche limit (see Fig. 11). Hence, if such discs are formed at the right time and if they are massive enough, recycled planets may be produced around this class of hosts, although stellar tides may impede their survival around $\mathrm{M}$ dwarfs. We have examined whether any known (candidate) exoplanets around the above types of objects may have formed via the recycling scenario, but find that none conclusively show the expected characteristics.

\section{ACKNOWLEDGEMENTS}

RvL, QK, MCW, and AS acknowledge support from the European Union through ERC grant number 279973. RvL is also supported by the DISCSIM project, grant agreement 341137 funded by the European Research Council under ERC-2013-ADG. QK acknowledges funding from STFC via the Institute of Astronomy, Cambridge Consolidated Grant. AS is partially supported by funding from the Center for Exoplanets and Habitable Worlds. The Center for Exoplanets and Habitable Worlds is supported by the Pennsylvania State University, the Eberly College of Science, and the Pennsylvania Space Grant Consortium.

\section{REFERENCES}

Agol E., 2011, ApJ, 731, L31

Althaus L. G., Córsico A. H., Isern J., García-Berro E., 2010, A\&ARv, 18, 471

Anglada-Escudé G., et al., 2016, Nature, 536, 437

Astudillo-Defru N., et al., 2017, A\&A, 605, L11

Baraffe I., Chabrier G., Allard F., Hauschildt P. H., 1998, A\&A, 337, 403

Baraffe I., Chabrier G., Barman T. S., Allard F., Hauschildt P. H., 2003, A\&A, 402, 701

Barnes R., Heller R., 2013, Astrobiology, 13, 279

Bear E., Soker N., 2012, ApJ, 749, L14

Bear E., Soker N., 2015, MNRAS, 450, 4233

Belardi C., Kilic M., Munn J. A., Gianninas A., Barber S. D., Dey A., Stetson P. B., 2016, MNRAS, 462, 2506

Berta-Thompson Z. K., et al., 2015, Nature, 527, 204

Bochkarev K. V., Rafikov R. R., 2011, ApJ, 741, 36

Bonsor A., Veras D., 2015, MNRAS, 454, 53

Bonsor A., Farihi J., Wyatt M. C., van Lieshout R., 2017, MNRAS, 468, 154

Bosh A. S., Olkin C. B., French R. G., Nicholson P. D., 2002, Icarus, 157, 57

Brahic A., 1977, A\&A, 54, 895

Burdanov A., Delrez L., Gillon M., Jehin E., 2017, preprint, (arXiv: 1710.03775) 
Burns J. A., Lamy P. L., Soter S., 1979, Icarus, 40, 1

Chambers J. E., Wetherill G. W., Boss A. P., 1996, Icarus, 119, 261

Chandrasekhar S., 1969, Ellipsoidal figures of equilibrium

Charnoz S., Salmon J., Crida A., 2010, Nature, 465, 752

Charnoz S., et al., 2011, Icarus, 216, 535

Charpinet S., et al., 2011, Nature, 480, 496

Colwell J. E., Nicholson P. D., Tiscareno M. S., Murray C. D., French R. G., Marouf E. A., 2009, The Structure of Saturn's Rings. p. 375, doi:10.1007/978-1-4020-9217-6_13

Cordes J. M., 1993, in Phillips J. A., Thorsett S. E., Kulkarni S. R., eds, Astronomical Society of the Pacific Conference Series Vol. 36, Planets Around Pulsars. pp 43-60

Crida A., Charnoz S., 2012, Science, 338, 1196

Croll B., et al., 2017, ApJ, 836, 82

Currie T., Hansen B., 2007, ApJ, 666, 1232

Cuzzi J. N., Durisen R. H., Burns J. A., Hamill P., 1979, Icarus, 38, 54

Cuzzi J., Clark R., Filacchione G., French R., Johnson R., Marouf E., Spilker L., 2009, Ring Particle Composition and Size Distribution. p. 459, doi:10.1007/978-1-4020-9217-6_15

Daisaka H., Tanaka H., Ida S., 2001, Icarus, 154, 296

Dermott S. F., 1984, in Greenberg R., Brahic A., eds, IAU Colloq. 75: Planetary Rings. pp 589-637

Dittmann J. A., et al., 2017, Nature, 544, 333

Dones L., 1991, Icarus, 92, 194

Dufour P., Kilic M., Fontaine G., Bergeron P., Melis C., Bochanski J., 2012, ApJ, 749, 6

Faedi F., West R. G., Burleigh M. R., Goad M. R., Hebb L., 2011, MNRAS, 410,899

Farihi J., 2016, New Astron. Rev., 71, 9

Farihi J., Zuckerman B., Becklin E. E., 2008a, ApJ, 674, 431

Farihi J., Becklin E. E., Zuckerman B., 2008b, ApJ, 681, 1470

Farihi J., Jura M., Zuckerman B., 2009, ApJ, 694, 805

Farihi J., Gänsicke B. T., Koester D., 2013, Science, 342, 218

Farihi J., von Hippel T., Pringle J. E., 2017, MNRAS, 471, L145

Farihi J., et al., 2018, MNRAS, 474, 947

Ferrario L., de Martino D., Gänsicke B. T., 2015, Space Sci. Rev., 191, 111

Fontaine G., Brassard P., Charpinet S., Green E. M., Randall S. K., Van Grootel V., 2012, A\&A, 539, A12

Friedjung M., 1985, A\&A, 146, 366

Friedrich S., Koester D., Christlieb N., Reimers D., Wisotzki L., 2000, A\&A, 363, 1040

Fulton B. J., et al., 2014, ApJ, 796, 114

Gänsicke B. T., Marsh T. R., Southworth J., Rebassa-Mansergas A., 2006, Science, 314, 1908

Gänsicke B. T., Koester D., Farihi J., Girven J., Parsons S. G., Breedt E., 2012, MNRAS, 424, 333

Gänsicke B. T., et al., 2016, ApJ, 818, L7

Gary B. L., Rappaport S., Kaye T. G., Alonso R., Hambschs F.-J., 2017, MNRAS, 465,3267

Gentile Fusillo N. P., Gänsicke B. T., Farihi J., Koester D., Schreiber M. R., Pala A. F., 2017, MNRAS, 468, 971

Gillon M., et al., 2016, Nature, 533, 221

Girven J., Brinkworth C. S., Farihi J., Gänsicke B. T., Hoard D. W., Marsh T. R., Koester D., 2012, ApJ, 749, 154

Goldreich P., Tremaine S. D., 1978, Icarus, 34, 240

Goldreich P., Tremaine S., 1980, ApJ, 241, 425

Goldreich P., Ward W. R., 1973, ApJ, 183, 1051

Graham J. R., Matthews K., Neugebauer G., Soifer B. T., 1990, ApJ, 357, 216

Gurri P., Veras D., Gänsicke B. T., 2017, MNRAS, 464, 321

Hahn J., 2009, The Dynamics of Planetary Systems and Astrophysical Disks. Wiley VCH Verlag GmbH, https://books.google.co.uk/ books?id=5BEAPgAACAA J

Hamers A. S., Portegies Zwart S. F., 2016, MNRAS, 462, L84

Harris A. W., 1996, Earth Moon and Planets, 72, 113

He M. Y., Triaud A. H. M. J., Gillon M., 2017, MNRAS, 464, 2687

Heber U., 2009, ARA\&A, 47, 211

Heber U., 2016, PASP, 128, 082001
Holsapple K. A., Michel P., 2006, Icarus, 183, 331

Holsapple K. A., Michel P., 2008, Icarus, 193, 283

Howell S. B., et al., 2014, PASP, 126, 398

Hyodo R., Charnoz S., 2017, AJ, 154, 34

Hyodo R., Ohtsuki K., 2015, Nature Geoscience, 8, 686

Hyodo R., Ohtsuki K., Takeda T., 2015, ApJ, 799, 40

Hyodo R., Charnoz S., Ohtsuki K., Genda H., 2017, Icarus, 282, 195

Irwin J., Berta Z. K., Burke C. J., Charbonneau D., Nutzman P., West A. A., Falco E. E., 2011, ApJ, 727, 56

Julian W. H., Toomre A., 1966, ApJ, 146, 810

Jura M., 2003, ApJ, 584, L91

Jura M., 2008, AJ, 135, 1785

Jura M., Young E. D., 2014, Annual Review of Earth and Planetary Sciences, 42,45

Jura M., Farihi J., Zuckerman B., Becklin E. E., 2007a, AJ, 133, 1927

Jura M., Farihi J., Zuckerman B., 2007b, ApJ, 663, 1285

Jura M., Farihi J., Zuckerman B., 2009a, AJ, 137, 3191

Jura M., Muno M. P., Farihi J., Zuckerman B., 2009b, ApJ, 699, 1473

Karjalainen R., 2007, Icarus, 189, 523

Kenyon S. J., Bromley B. C., 2017a, ApJ, 844, 116

Kenyon S. J., Bromley B. C., 2017b, ApJ, 850, 50

Kleinman S. J., et al., 2013, ApJS, 204, 5

Koester D., 2009, A\&A, 498, 517

Konacki M., Wolszczan A., 2003, ApJ, 591, L147

Kral Q., Wyatt M. C., Triaud A. H. M. J., Marino S., Thebault P., Shorttle O., 2018, preprint, (arXiv: 1802.05034)

Krzesinski J., 2015, A\&A, 581, A7

Law N. M., et al., 2015, PASP, 127, 234

Li J., Ferrario L., Wickramasinghe D., 1998, ApJ, 503, L151

Lin H. W., Loeb A., 2014, ApJ, 793, L43

Lin D. N. C., Pringle J. E., 1987, MNRAS, 225, 607

Loeb A., Maoz D., 2013, MNRAS, 432, L11

Margalit B., Metzger B. D., 2017, MNRAS, 465, 2790

Melis C., Jura M., Albert L., Klein B., Zuckerman B., 2010, ApJ, 722, 1078

Metchev S. A., et al., 2015, ApJ, 799, 154

Metzger B. D., Rafikov R. R., Bochkarev K. V., 2012, MNRAS, 423, 505

Meyer-Vernet N., Sicardy B., 1987, Icarus, 69, 157

Michikoshi S., Inutsuka S.-i., Kokubo E., Furuya I., 2007, ApJ, 657, 521

Michikoshi S., Kokubo E., Inutsuka S.-i., 2009, ApJ, 703, 1363

Muirhead P. S., et al., 2012, ApJ, 747, 144

Mustill A. J., Villaver E., 2012, ApJ, 761, 121

Mustill A. J., Veras D., Villaver E., 2014, MNRAS, 437, 1404

Nauenberg M., 1972, ApJ, 175, 417

Nelson A. F., 2006, MNRAS, 373, 1039

Nordhaus J., Spiegel D. S., 2013, MNRAS, 432, 500

Nutzman P., Charbonneau D., 2008, PASP, 120, 317

Ohtsuki K., 1999, Icarus, 137, 152

Passy J.-C., Mac Low M.-M., De Marco O., 2012, ApJ, 759, L30

Payne M. J., Veras D., Holman M. J., Gänsicke B. T., 2016, MNRAS, 457, 217

Payne M. J., Veras D., Gänsicke B. T., Holman M. J., 2017, MNRAS, 464, 2557

Phinney E. S., Hansen B. M. S., 1993, in Phillips J. A., Thorsett S. E., Kulkarni S. R., eds, Astronomical Society of the Pacific Conference Series Vol. 36, Planets Around Pulsars. pp 371-390

Porco C. C., Thomas P. C., Weiss J. W., Richardson D. C., 2007, Science, 318,1602

Pringle J. E., 1981, ARA\&A, 19, 137

Pringle J. E., 1991, MNRAS, 248, 754

Raddi R., Gänsicke B. T., Koester D., Farihi J., Hermes J. J., Scaringi S., Breedt E., Girven J., 2015, MNRAS, 450, 2083

Raddi R., et al., 2017, MNRAS, 472, 4173

Radigan J., Lafrenière D., Jayawardhana R., Artigau E., 2014, ApJ, 793, 75

Rafikov R. R., 2011a, MNRAS, 416, L55

Rafikov R. R., 2011b, ApJ, 732, L3

Rafikov R. R., 2018, preprint, (arXiv: 1801.02658)

Rafikov R. R., Garmilla J. A., 2012, ApJ, 760, 123 
Rappaport S., Barclay T., DeVore J., Rowe J., Sanchis-Ojeda R., Still M., 2014, ApJ, 784, 40

Rappaport S., Gary B. L., Kaye T., Vanderburg A., Croll B., Benni P., Foote J., 2016, MNRAS, 458, 3904

Rappaport S., Gary B. L., Vanderburg A., Xu S., Pooley D., Mukai K., 2018, MNRAS, 474, 933

Rauer H., et al., 2014, Experimental Astronomy, 38, 249

Redfield S., Farihi J., Cauley P. W., Parsons S. G., Gänsicke B. T., Duvvuri G. M., 2017, ApJ, 839, 42

Ricker G. R., et al., 2015, Journal of Astronomical Telescopes, Instruments, and Systems, 1, 014003

Roche É., 1849, Académie des sciences et des lettres de Montpellier: Mémoire de la section des sciences, 1, 243

Rosenblatt P., Charnoz S., Dunseath K. M., Terao-Dunseath M., Trinh A., Hyodo R., Genda H., Toupin S., 2016, Nature Geoscience, 9, 581

Rowe J. F., et al., 2014, ApJ, 784, 45

Salmon J., Canup R. M., 2017, ApJ, 836, 109

Salmon J., Charnoz S., Crida A., Brahic A., 2010, Icarus, 209, 771

Salo H., 1992, Nature, 359, 619

Salo H., 1995, Icarus, 117, 287

Salo H., 2012, Progress of Theoretical Physics Supplement, 195, 48

Sandhaus P. H., Debes J. H., Ely J., Hines D. C., Bourque M., 2016, ApJ, 823,49

Schlichting H. E., Mukhopadhyay S., 2018, Space Sci. Rev., 214, 34

Schmidt J., Ohtsuki K., Rappaport N., Salo H., Spahn F., 2009, Dynamics of Saturn's Dense Rings. p. 413, doi:10.1007/978-1-4020-9217-6_14

Shannon R. M., et al., 2013, ApJ, 766, 5

Shukhman I. G., 1984, Soviet Ast., 28, 574

Shuvalov V., 2009, Meteoritics and Planetary Science, 44, 1095

Silvotti R., et al., 2014, A\&A, 570, A130

Stassun K. G., et al., 2017, preprint, (arXiv: 1706.00495)

Stephan A. P., Naoz S., Zuckerman B., 2017, ApJ, 844, L16

Tajeddine R., Nicholson P. D., Longaretti P.-Y., El Moutamid M., Burns J. A., 2017, ApJS, 232, 28

Takeuchi T., Miyama S. M., Lin D. N. C., 1996, ApJ, 460, 832

Thorsett S. E., Dewey R. J., 1993, ApJ, 419, L65

Thorsett S. E., Phillips J. A., 1992, ApJ, 387, L69

Tiscareno M. S., Hedman M. M., Burns J. A., Castillo-Rogez J., 2013, ApJ, $765, \mathrm{~L} 28$

Toomre A., 1964, ApJ, 139, 1217

Van Grootel V., Charpinet S., Fontaine G., Green E. M., Brassard P., 2010, A\&A, 524, A63

Vanderburg A., et al., 2015, Nature, 526, 546

Veras D., 2016, Royal Society Open Science, 3, 150571

Veras D., Gänsicke B. T., 2015, MNRAS, 447, 1049

Veras D., Leinhardt Z. M., Bonsor A., Gänsicke B. T., 2014a, MNRAS, 445, 2244

Veras D., Shannon A., Gänsicke B. T., 2014b, MNRAS, 445, 4175

Veras D., Leinhardt Z. M., Eggl S., Gänsicke B. T., 2015, MNRAS, 451, 3453

Veras D., Mustill A. J., Gänsicke B. T., Redfield S., Georgakarakos N., Bowler A. B., Lloyd M. J. S., 2016a, MNRAS, 458, 3942

Veras D., Marsh T. R., Gänsicke B. T., 2016b, MNRAS, 461, 1413

Veras D., Carter P. J., Leinhardt Z. M., Gänsicke B. T., 2017, MNRAS, 465, 1008

Wallach A., et al., 2018, preprint, (arXiv: 1803.03584)

Wang Z., Chakrabarty D., Kaplan D. L., 2006, Nature, 440, 772

Wickramasinghe D. T., Farihi J., Tout C. A., Ferrario L., Stancliffe R. J., 2010, MNRAS, 404, 1984

Willes A. J., Wu K., 2004, MNRAS, 348, 285

Willes A. J., Wu K., 2005, A\&A, 432, 1091

Wisdom J., Tremaine S., 1988, AJ, 95, 925

Wolszczan A., 1994, Science, 264, 538

Wolszczan A., Frail D. A., 1992, Nature, 355, 145

Xu S., Jura M., Dufour P., Zuckerman B., 2016, ApJ, 816, L22

Xu S., Zuckerman B., Dufour P., Young E. D., Klein B., Jura M., 2017, ApJ, 836, L7

Xu S., et al., 2018, MNRAS, 474, 4795
Yasui Y., Ohtsuki K., Daisaka H., 2012, AJ, 143, 110

Zhou G., et al., 2016, MNRAS, 463, 4422

Zuckerman B., Melis C., Klein B., Koester D., Jura M., 2010, ApJ, 722, 725 van Sluijs L., Van Eylen V., 2018, MNRAS, 474, 4603

\section{APPENDIX A: TORQUE-DENSITY BALANCE}

Viscous spreading of a disc leads to the outward transport of angular momentum, while PR drag acts to remove angular momentum. Since angular-momentum change rates are equivalent to torques, the conditions under which these two processes are in balance can be found by equating their radial torque densities:

$\frac{\mathrm{d} \Gamma_{\text {visc }}}{\mathrm{d} r}=-\frac{\mathrm{d} \Gamma_{\mathrm{PR}}}{\mathrm{d} r}$.

We now proceed to determine expressions for the individual torque densities associated with viscosity and PR drag.

At any point in the disc, the viscous torque of the material inside radius $r$ on material outside $r$ can be written as (Pringle 1981)

$\Gamma_{\text {visc }}=2 \pi r \times v \Sigma A \times r, \quad A=-r \frac{\mathrm{d} \Omega_{\mathrm{K}}}{\mathrm{d} r}=\frac{3}{2} \Omega_{\mathrm{K}}$,

where $A$ is the local shearing rate. Inserting the viscosity prescription for a gravitationally unstable particle disc (Eq. (13)) and taking the radial derivative (assuming the surface density is constant with radius) gives the viscous torque density

$$
\frac{\mathrm{d} \Gamma_{\text {visc }}}{\mathrm{d} r} \simeq 30 \pi \Sigma v r \Omega_{\mathrm{K}} \simeq 780 \pi r_{\mathrm{H}}^{* 5} \frac{G^{2} \Sigma^{3} r}{\Omega_{\mathrm{K}}^{2}} \text {. }
$$

To find the PR-drag torque density, we consider the azimuthal force per unit area exerted on the disc by the impinging stellar radiation through PR drag (Rafikov 2011b)

$f_{\varphi, \mathrm{PR}}=-\zeta \frac{L_{\star} \phi_{r}}{4 \pi r^{2} c} \frac{\Omega_{\mathrm{K}} r}{c}, \quad \phi_{r} \simeq 1-\exp \left(-\tau_{\|}\right)$.

Here, $\phi_{r}$ characterises the efficiency of radiative momentum absorption by the disc surface. For a disc that is optically thick to the impinging radiation $\left(\tau_{\|} \gg 1\right)$, this factor simplifies to $\phi_{r} \simeq 1$. We choose the negative sign because the azimuthal PR-drag force acts in the direction opposite to the rotation of the disc. The PR-drag torque on a narrow disc annulus of width $\mathrm{d} r$ is $2 \pi r \mathrm{~d} r \times f_{\varphi, \mathrm{PR}} \times r$, and hence the radial density of PR-drag torque on the disc is

$\frac{\mathrm{d} \Gamma_{\mathrm{PR}}}{\mathrm{d} r}=2 \pi r \times f_{\varphi, \mathrm{PR}} \times r=-\frac{\zeta \phi_{r}}{2} \frac{L_{\star}}{c^{2}} \Omega_{\mathrm{K}} r$.

Inserting expressions (A3) and (A5) into Eq. (A1), using $\phi_{r} \simeq$ 1 , and solving for $\Sigma$ gives the critical surface density

$\Sigma_{\text {crit }} \simeq\left(\frac{\zeta L_{\star}}{1560 \pi c^{2} G^{2} r_{\mathrm{H}}^{* 5}}\right)^{1 / 3} \Omega_{\mathrm{K}}$.

This result can be rewritten to produce Eq. (17) multiplied by a factor $(2 / 5)^{1 / 3} \approx 0.74$.

This paper has been typeset from a $\mathrm{T}_{\mathrm{E}} \mathrm{X} / \mathrm{L} \mathrm{T} \mathrm{E}$ file prepared by the author. 